\title{
Stereoselective Multimodal Transformation of Planar Chiral 9-Membered Diallylic Amides
}

\author{
Katsuhiko Tomooka, ${ }^{* \dagger}$ Masaki Suzuki, ${ }^{*}$ Maki Shimada, ${ }^{*}$ Runyan Ni, ${ }^{\S}$ and Kazuhiro Uehara ${ }^{\dagger}$ \\ ${ }^{\dagger}$ Institute for Materials Chemistry and Engineering, Kyushu University, \\ 6-1 Kasuga-koen, Kasuga-shi, Fukuoka 816-8580, Japan, \\ ${ }^{\dagger}$ Department of Applied Chemistry, Graduate School of Science and Engineering, \\ Tokyo Institute of Technology, \\ 2-12-1 O-okayama, Meguro-ku, Tokyo 152-8552, Japan, and \\ ${ }^{\S}$ Interdisciplinary Graduate School of Engineering Sciences, Kyushu University, \\ 6-1 Kasuga-koen, Kasuga-shi, Fukuoka 816-8580, Japan \\ ktomooka@cm.kyushu-u.ac.jp
}

\section{Table of Contents:}

General Experimental

$\mathrm{S} 2$

Detailed Reaction Procedures and Analytical Data

${ }^{1} \mathrm{H},{ }^{13} \mathrm{C},{ }^{19} \mathrm{~F}$ and ${ }^{31} \mathrm{P}$ NMR Spectra

S20-S77 


\section{General Experimental}

All reactions were carried out in heat-gun-dried glassware under an argon atmosphere unless otherwise noted. Dry $\mathrm{Et}_{2} \mathrm{O}, \mathrm{CH}_{2} \mathrm{Cl}_{2}$ and $\mathrm{CH}_{3} \mathrm{CN}$ were purchased from Kanto Chemical Co., Inc. and used without purification. ${ }^{1} \mathrm{H} \mathrm{NMR},{ }^{13} \mathrm{C} \mathrm{NMR},{ }^{19} \mathrm{~F}$ NMR and ${ }^{31} \mathrm{P}$ NMR spectra were recorded on a Varian Gemine-2000 $\left({ }^{1} \mathrm{H}, 300 \mathrm{MHz} ;{ }^{13} \mathrm{C}, 75 \mathrm{MHz}\right)$, a Varian Mercury $\left({ }^{1} \mathrm{H}, 300 \mathrm{MHz} ;{ }^{13} \mathrm{C}, 75 \mathrm{MHz}\right)$ or a JEOL Lambda $600\left({ }^{19} \mathrm{~F}, 565 \mathrm{MHz} ;{ }^{31} \mathrm{P}, 243 \mathrm{MHz}\right)$ at ambient temperature using $\mathrm{CDCl}_{3}$ as solvents. Chemical Shifts $\delta$ in ppm were referenced to the solvent residual peak as an internal standard $\mathrm{CHCl}_{3}$ for ${ }^{1} \mathrm{H} \mathrm{NMR}\left(\delta\right.$ 7.26), to the solvent signal as $\mathrm{CDCl}_{3}$ for ${ }^{13} \mathrm{C} \mathrm{NMR}(\delta 77.1)$, and to the standard resonances as external $\mathrm{C}_{6} \mathrm{~F}_{6}$ for ${ }^{19} \mathrm{~F}$ NMR $(\delta-164.9)$ and external $\mathrm{H}_{3} \mathrm{PO}_{4}$ for ${ }^{31} \mathrm{P}$ NMR ( $\delta$ 0.00). The peak multiplicities were given as followed: s, singlet; $\mathrm{d}$, doublet; q, quartet; $\mathrm{m}$, multiplet; br, broad. Optical rotations were measured on a digital polarimeter (JASCO DIP-370). Infrared spectra were recorded on a Fourier transfer infrared spectrophotometer (Perkin Elmer SpectrumOne) as neat liquid on $\mathrm{NaCl}$ plates and as crystal used a diffuse reflector. Melting points (Mp) were measured on a Yanaco Micro Melting Point Apparatus. High performance liquid chromatography (HPLC) was performed on a SHIMADZU SPD-6A UV detector equipped with a SHIMADZU LC-9A or JASCO UV-2075 and CD-2095 detectors equipped with JASCO PU-2087 or PU-2089 using Kanto Mightysil Si $60(5 \mu \mathrm{m})(4.6 \mathrm{~mm} \times 15 \mathrm{~cm}),(2.0 \mathrm{~cm} \times 25 \mathrm{~cm})$, Daicel CHIRALCEL OD-H column $(4.6 \mathrm{~mm} \times 25 \mathrm{~cm}),(2.0 \mathrm{~cm} \times 25 \mathrm{~cm})$, Daicel CHIRALPAK AD-H column $(4.6 \mathrm{~mm} \times 25 \mathrm{~cm})$, or Daicel CHIRALPAK AS-H column $(4.6 \mathrm{~mm} \times 25 \mathrm{~cm}),(2.0 \mathrm{~cm} \times 25$ $\mathrm{cm})$. Analytical thin-layer chromatography (TLC) was carried out on silica gel $60 \mathrm{~F}_{254}$ (Merck 5715) plates and developed plates were visualized by UV $(254 \mathrm{~nm})$ and by heating on a hot plate after staining with a $4 \%$ solution of phosphomolybdic acid in ethanol or a $2.5 \%$ solution of $p$-anisaldehyde in ethanol. Column chromatography was performed using Kanto spherical silica gel $60 \mathrm{~N}(63-210 \mu \mathrm{m})$ or Fuji Silysia silica gel FL100D (neutral). X-ray crystallographic data was collected using a Rigaku Saturn CCD diffractometer with graphite monochromated Mo-K $\alpha$ radiation $(\lambda=0.7107 \AA)$ at $123 \mathrm{~K}$ or a Rigaku-IP diffractometer with $\mathrm{Cu}-\mathrm{K} \alpha$ radiation $(\lambda=1.5418 \AA)$ at 113 K. HRMS analyses were performed at the Analytical Center in IMCE, Kyushu University. Elemental analyses were performed by the Elemental Analysis Center, Faculty of Science, Kyushu University and by the Analytical Facility at the Research Laboratory of Resources Utilization, Tokyo Institute of Technology. 


\section{Detailed Reaction Procedures and Analytical Data}

\section{1) Epoxidation of cyclic amides 1a}

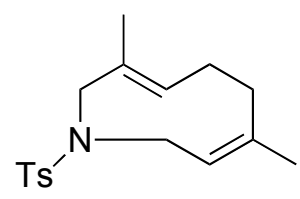

$(S)-1 \mathbf{a}$ $(>98 \%$ ee $)$

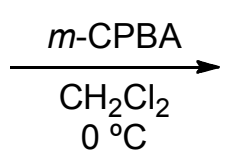

$(3 R, 4 R)-2 \mathbf{a}$

$(>98 \% \mathrm{dr},>98 \%$ ee $)$

$64 \%$

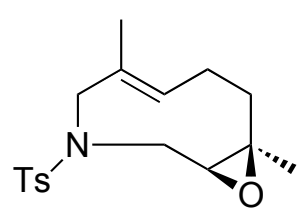

$(7 R, 8 S)-3 a$ $(>98 \% \mathrm{dr},>98 \%$ ee $)$

$10 \%$

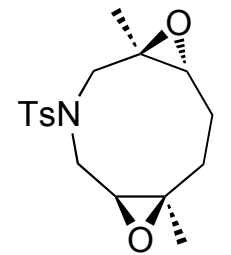

$(3 R, 4 R, 7 R, 8 S)-\mathbf{4 a}$ $(>98 \% \mathrm{dr},>98 \%$ ee)

$24 \%$

To a solution of $(S)-1 \mathrm{a}(>98 \%$ ee; $35.3 \mathrm{mg}, 0.116 \mathrm{mmol})$ in dry $\mathrm{CH}_{2} \mathrm{Cl}_{2}(7 \mathrm{~mL})$ at $0{ }^{\circ} \mathrm{C}$ was added $m$-CPBA (24.9 mg, $0.144 \mathrm{mmol})$. The reaction mixture was stirred at that temperature for $4 \mathrm{~h}$. The reaction was quenched with saturated aqueous $\mathrm{Na}_{2} \mathrm{~S}_{2} \mathrm{O}_{3}$ and saturated aqueous $\mathrm{NaHCO}_{3}$. The phases were separated and the aqueous layer was extracted with $\mathrm{CH}_{2} \mathrm{Cl}_{2}$. The combined organic phases were dried over $\mathrm{Na}_{2} \mathrm{SO}_{4}$, filtered and the solvent was removed in vacuo. The residue was purified by silica gel chromatography (hexane/AcOEt $=20 / 1$ to $2 / 1$ ) to afford $27.7 \mathrm{mg}$ of a mixture of mono-epoxides 2a and 3a (74\%; 2a/3a $=87 / 13$ determined by ${ }^{1} \mathrm{H}$ NMR analysis) as a white solid and $9.7 \mathrm{mg}$ (24\%) of $\mathbf{4 a}$ as a white solid. The mixture of $\mathbf{2 a}$ and 3a were separated by silica gel HPLC; column: Kanto Mightysil Si $60(5 \mu \mathrm{m})(2.0 \mathrm{~cm} \times 25 \mathrm{~cm})$, eluent: hexane/AcOEt $=4 / 1$, flow rate: $7.0 \mathrm{~mL} / \mathrm{min}$, detection: UV $254 \mathrm{~nm}$, temperature: $\mathrm{rt}$. All of epoxides $(3 R, 4 R)-\mathbf{2 a},(7 R, 8 S)-\mathbf{3 a}$ and $(3 R, 4 R, 7 R, 8 S)-4 \mathbf{a}$ were determined as a single stereoisomer $\left(>98 \% \mathrm{dr}\right.$ analyzed by ${ }^{1} \mathrm{H} \mathrm{NMR}$, $>98 \%$ ee analyzed by chiral HPLC).

\section{4,9-dimethyl-7-(p-toluenesulfonyl)-10-oxa-7-azabicyclo[7.1.0]dec-4-ene [(3R,4R)-2a]}

${ }^{1} \mathrm{H}$ NMR $\left(300 \mathrm{MHz}, \mathrm{CDCl}_{3}\right): \delta 7.62(\mathrm{~d}, J=8.3 \mathrm{~Hz}, 2 \mathrm{H}), 7.28(\mathrm{~d}, J=8.1 \mathrm{~Hz}, 2 \mathrm{H}), 5.46(\mathrm{dd}, J=11.7$, $4.7 \mathrm{~Hz}, 1 \mathrm{H}), 4.17(\mathrm{~d}, J=11.3 \mathrm{~Hz}, 1 \mathrm{H}), 4.00(\mathrm{dd}, J=15.0,4.7 \mathrm{~Hz}, 1 \mathrm{H}), 3.26(\mathrm{dd}, J=15.0,11.7 \mathrm{~Hz}$, $1 \mathrm{H}), 2.77(\mathrm{dd}, J=11.8,2.7 \mathrm{~Hz}, 1 \mathrm{H}), 2.41(\mathrm{~s}, 3 \mathrm{H}), 2.41-2.31(\mathrm{~m}, 1 \mathrm{H}), 2.11(\mathrm{~d}, J=11.3 \mathrm{~Hz}, 1 \mathrm{H})$, 2.06-1.95 (m, 2H), $1.75(\mathrm{~s}, 3 \mathrm{H}), 1.23(\mathrm{~s}, 3 \mathrm{H}), 1.21-1.07(\mathrm{~m}, 1 \mathrm{H}) ;{ }^{13} \mathrm{C} \mathrm{NMR}\left(75 \mathrm{MHz}, \mathrm{CDCl}_{3}\right): \delta$ 143.3, 135.9, 135.4, 129.7, 126.8, 124.4, 66.3, 57.5, 57.0, 45.7, 27.9, 25.5, 23.1, 21.6, 16.6; IR (neat, $\mathrm{cm}^{-1}$ ): 2935, 1917, 1598, 1461, 1323, 1236, 1091, 812, 658, 550, 510; Mp: $142{ }^{\circ} \mathrm{C}$; Analytical HPLC [column: CHIRALCEL OD-H $(4.6 \mathrm{~mm} \times 25 \mathrm{~cm})$, eluent: hexane $/ i$-PrOH $=2 / 1$, flow rate: 0.5 $\mathrm{mL} / \mathrm{min}$, detection: UV $254 \mathrm{~nm}$, temperature: $\mathrm{rt}] t_{R}=14.9 \mathrm{~min}$ for $(-)-(3 R, 4 R)$-isomer, $23.7 \mathrm{~min}$ for $(+)-(3 S, 4 S)$-isomer; Optical rotation value: $[\alpha]_{\mathrm{D}}{ }^{28}-49.6\left(c 1.03, \mathrm{CHCl}_{3}\right)$ for $(3 R, 4 R)$-isomer $(>98 \%$ ee); HRMS (EI, positive): $m / z$ calcd for $\mathrm{C}_{17} \mathrm{H}_{23} \mathrm{NO}_{3} \mathrm{~S}[\mathrm{M}]^{+}: 321.1399$, found: 321.1396; Anal. calcd for $\mathrm{C}_{17} \mathrm{H}_{23} \mathrm{NO}_{3} \mathrm{~S}$ : C, 63.52; H, 7.21; N, 4.36; S, 9.98. Found: C, 63.32; H, 7.07; N, 4.15; S, 9.86.

\section{5,9-dimethyl-3-(p-toluenesulfonyl)-10-oxa-3-azabicyclo[7.1.0]dec-5-ene [(7R,8S)-3a]}

${ }^{1} \mathrm{H}$ NMR $\left(300 \mathrm{MHz}, \mathrm{CDCl}_{3}\right): \delta 7.67(\mathrm{~d}, J=8.1 \mathrm{~Hz}, 2 \mathrm{H}), 7.31(\mathrm{~d}, J=8.1 \mathrm{~Hz}, 2 \mathrm{H}), 5.24(\mathrm{dd}, J=11.4$, $3.6 \mathrm{~Hz}, 1 \mathrm{H}), 4.33(\mathrm{~d}, J=10.5 \mathrm{~Hz}, 1 \mathrm{H}), 3.84(\mathrm{dd}, J=15.0,2.1 \mathrm{~Hz}, 1 \mathrm{H}), 2.96(\mathrm{~d}, J=10.5 \mathrm{~Hz}, 1 \mathrm{H})$, $2.84(\mathrm{dd}, J=10.2,2.1 \mathrm{~Hz}, 1 \mathrm{H}), 2.43(\mathrm{~s}, 3 \mathrm{H}), 2.41-2.29(\mathrm{~m}, 1 \mathrm{H}), 2.28(\mathrm{dd}, J=15.0,10.2 \mathrm{~Hz}, 1 \mathrm{H})$, 2.17-2.04 (m, 2H), $1.74(\mathrm{~s}, 3 \mathrm{H}), 1.34(\mathrm{~s}, 3 \mathrm{H}), 1.10-1.01(\mathrm{~m}, 1 \mathrm{H}) ;{ }^{13} \mathrm{C} \mathrm{NMR}\left(75 \mathrm{MHz}, \mathrm{CDCl}_{3}\right): \delta$ 143.3, 135.8, 132.7, 129.8, 129.7, 127.0, 64.8, 61.9, 58.5, 45.5, 34.3, 25.4, 22.9, 21.7, 15.6; IR (neat, $\mathrm{cm}^{-1}$ ): $2925,2862,1458,1346,1331,1010,850,658,548$; Mp: $154{ }^{\circ} \mathrm{C}$; Analytical HPLC [column: CHIRALCEL OD-H $(4.6 \mathrm{~mm} \times 25 \mathrm{~cm})$, eluent: hexane $/ i$-PrOH $=2 / 1$, flow rate: $0.5 \mathrm{~mL} / \mathrm{min}$, detection: UV $254 \mathrm{~nm}$, temperature: $\mathrm{rt}] t_{R}=21.3 \mathrm{~min}$ for $(-)-(7 S, 8 R)$-isomer, $38.0 \mathrm{~min}$ for 
$(+)-(7 R, 8 S)$-isomer; Optical rotation value: $[\alpha]_{\mathrm{D}}{ }^{27} 82.7(c 0.28, \mathrm{EtOH})$ for $(7 R, 8 S)$-isomer $(>98 \%$ ee); HRMS (FAB, matrix: 3-nitrobenzyl alcohol, positive): $m / z$ calcd for $\mathrm{C}_{17} \mathrm{H}_{24} \mathrm{NO}_{3} \mathrm{~S}[\mathrm{M}+\mathrm{H}]^{+}: 322.1477$, found: 322.1470; Anal. calcd for $\mathrm{C}_{17} \mathrm{H}_{23} \mathrm{NO}_{3} \mathrm{~S}$ : C, 63.52; H, 7.21; N, 4.36; S, 9.98. Found: C, 63.57; H, 7.35; N, 4.49; S, 9.90.

\section{1,6-dimethyl-8-(p-toluenesulfonyl)-5,11-dioxa-8-azatricyclo $\left[8.1 .0 .0^{4,6}\right]$ undecane [(3R,4R,7R,8S)-4a]}

${ }^{1} \mathrm{H}$ NMR $\left(300 \mathrm{MHz}, \mathrm{CDCl}_{3}\right): \delta 7.66(\mathrm{~d}, J=8.1 \mathrm{~Hz}, 2 \mathrm{H}), 7.32(\mathrm{~d}, J=8.1 \mathrm{~Hz}, 2 \mathrm{H}), 4.27(\mathrm{~d}, J=11.4$ $\mathrm{Hz}, 1 \mathrm{H}), 4.01(\mathrm{dd}, J=15.3,1.7 \mathrm{~Hz}, 1 \mathrm{H}), 3.11(\mathrm{dd}, J=10.4,1.7 \mathrm{~Hz}, 1 \mathrm{H}), 2.61(\mathrm{dd}, J=10.2,2.7 \mathrm{~Hz}$, $1 \mathrm{H}), 2.49(\mathrm{dd}, J=15.3,10.4 \mathrm{~Hz}, 1 \mathrm{H}), 2.43(\mathrm{~s}, 3 \mathrm{H}), 2.25-2.13(\mathrm{~m}, 2 \mathrm{H}), 2.09(\mathrm{~d}, J=11.4 \mathrm{~Hz}, 1 \mathrm{H})$, 1.50-1.37 (m, 2H), $1.44(\mathrm{~s}, 3 \mathrm{H}), 1.37(\mathrm{~s}, 3 \mathrm{H}) ;{ }^{13} \mathrm{C} \mathrm{NMR}\left(75 \mathrm{MHz}, \mathrm{CDCl}_{3}\right): \delta 143.8,135.3,129.9$, 127.0, 65.3, 64.4, 61.8, 57.3, 57.0, 46.0, 30.9, 23.5, 22.8, 21.7, 15.8; IR (neat, $\mathrm{cm}^{-1}$ ): 2927, 1462, 1349, 1335, 1164, 1079, 1021, 657, 571, 548; Mp: $215{ }^{\circ} \mathrm{C}$; Analytical HPLC [column: CHIRALPAK AD-H $(4.6 \mathrm{~mm} \times 25 \mathrm{~cm})$, eluent: hexane $/ i-\mathrm{PrOH}=1 / 1$, flow rate: $0.5 \mathrm{~mL} / \mathrm{min}$, detection: UV $254 \mathrm{~nm}$, temperature: $\mathrm{rt}] t_{R}=23.5 \mathrm{~min}$ for $(+)-(3 S, 4 S, 7 S, 8 R)$-isomer, $32.9 \mathrm{~min}$ for $(-)-(3 R, 4 R, 7 R, 8 S)$-isomer; Optical rotation value: $[\alpha]_{\mathrm{D}}{ }^{28}-39.8 \quad\left(c \quad 0.79, \mathrm{CHCl}_{3}\right)$ for $(3 R, 4 R, 7 R, 8 S)$-isomer ( $>98 \%$ ee); HRMS (FAB, matrix: 3-nitrobenzyl alcohol, positive): $\mathrm{m} / \mathrm{z}$ calcd for $\mathrm{C}_{17} \mathrm{H}_{24} \mathrm{NO}_{4} \mathrm{~S}[\mathrm{M}+\mathrm{H}]^{+}: 338.1426$, found: 338.1430; Anal. calcd for $\mathrm{C}_{17} \mathrm{H}_{23} \mathrm{NO}_{4} \mathrm{~S}$ : $\mathrm{C}, 60.51 ; \mathrm{H}$, 6.87; N, 4.15; S, 9.50. Found: C, 60.31; H, 6.76; N, 3.99; S, 9.31.

Recrystallization from hexane/ $\mathrm{CH}_{2} \mathrm{Cl}_{2} / \mathrm{AcOEt}$ afforded a single crystal that was suitable for X-ray analysis.

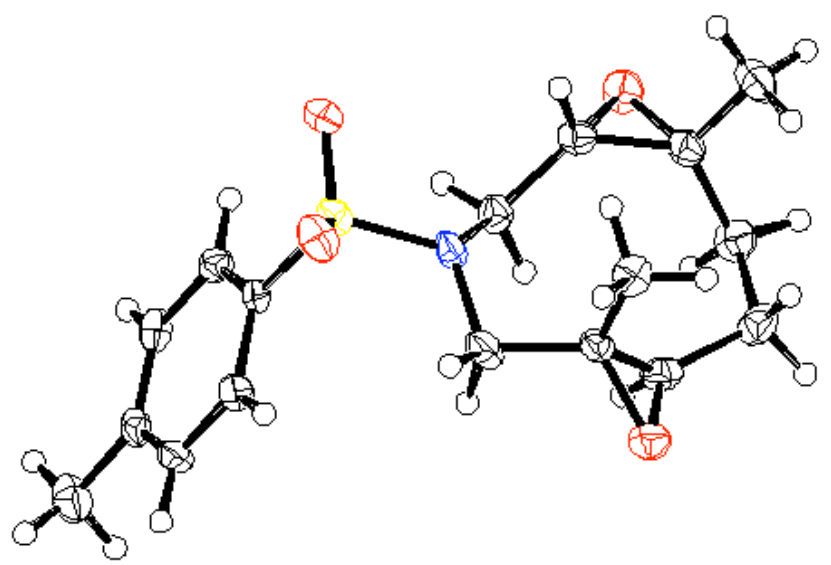

Figure S1. Molecular structure of $(3 R, 4 R, 7 R, 8 S)-\mathbf{4 a}$ (CCDC-817441) in the solid state. Thermal ellipsoids are drawn at the $50 \%$ probability level. 


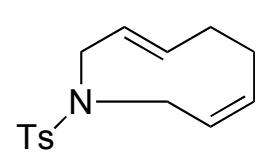

(S)-1b

$(>98 \%$ ee $)$

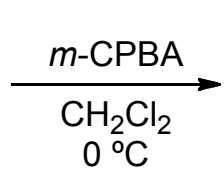

$(3 R, 4 R)-\mathbf{2 b}$ $(>98 \% \mathrm{dr},>98 \%$ ee)

$72 \%$

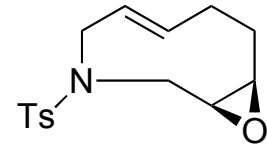

$(7 R, 8 S)-3 \mathbf{b}$ $(>98 \% \mathrm{dr},>98 \%$ ee) $11 \%$

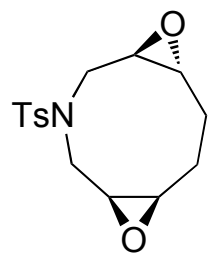

$(3 R, 4 R, 7 R, 8 S)-\mathbf{4 b}$ ( $>98 \% \mathrm{dr},>98 \%$ ee)

$11 \%$

To a solution of $(S)-\mathbf{1 b}(>98 \%$ ee; $50.0 \mathrm{mg}, 0.180 \mathrm{mmol})$ in dry $\mathrm{CH}_{2} \mathrm{Cl}_{2}(10 \mathrm{~mL})$ at $0{ }^{\circ} \mathrm{C}$ was added $m$-CPBA ( $40.4 \mathrm{mg}, 0.234 \mathrm{mmol}$ ). The reaction mixture was stirred at that temperature for 45 h. The reaction was quenched with saturated aqueous $\mathrm{Na}_{2} \mathrm{~S}_{2} \mathrm{O}_{3}$ and saturated aqueous $\mathrm{NaHCO}_{3}$. The phases were separated and the aqueous layer was extracted with $\mathrm{CH}_{2} \mathrm{Cl}_{2}$. The combined organic phases were dried over $\mathrm{Na}_{2} \mathrm{SO}_{4}$, filtered and the solvent was removed in vacuo. The residue was purified by silica gel chromatography (hexane/AcOEt $=1 / 0$ to $2 / 1$ ) to afford $3.0 \mathrm{mg}$ of the recovered starting material $(S)-\mathbf{1 b}(6 \%$; $>98 \%$ ee analyzed by HPLC), $44.0 \mathrm{mg}$ of a mixture of mono-epoxides $\mathbf{2 b}$ and $\mathbf{3 b}\left(83 \% ; \mathbf{2 b} / \mathbf{3 b}=86 / 14\right.$ determined by ${ }^{1} \mathrm{H}$ NMR analysis $)$ as a white solid and $6.1 \mathrm{mg}(11 \%)$ of $\mathbf{4 b}$ as a white solid. The mixture of $\mathbf{2 b}$ and $\mathbf{3 b}$ were separated by silica gel HPLC; column: Kanto Mightysil Si $60(5 \mu \mathrm{m})(2.0 \mathrm{~cm} \times 25 \mathrm{~cm})$, eluent: hexane/AcOEt $=4 / 1$, flow rate: $7.0 \mathrm{~mL} / \mathrm{min}$, detection: UV $254 \mathrm{~nm}$, temperature: rt. All of epoxides $(3 R, 4 R)-\mathbf{2 b}, \quad(7 R, 8 S)-\mathbf{3 b}$ and $(3 R, 4 R, 7 R, 8 S)-\mathbf{4 b}$ were determined as a single stereoisomer $\left(>98 \% \mathrm{dr}\right.$ analyzed by ${ }^{1} \mathrm{H} \mathrm{NMR},>98 \%$ ee analyzed by chiral HPLC).

\section{7-(p-toluenesulfonyl)-10-oxa-7-azabicyclo[7.1.0]dec-4-ene [(3R,4R)-2b]}

${ }^{1} \mathrm{H}$ NMR $\left(300 \mathrm{MHz}, \mathrm{CDCl}_{3}\right): \delta 7.66(\mathrm{~d}, J=8.0 \mathrm{~Hz}, 2 \mathrm{H}), 7.31(\mathrm{~d}, J=8.0 \mathrm{~Hz}, 2 \mathrm{H}), 5.72(\mathrm{ddd}, J=11.4$, $11.1,4.5 \mathrm{~Hz}, 1 \mathrm{H}), 5.65-5.55(\mathrm{~m}, 1 \mathrm{H}), 4.34(\mathrm{dd}, J=11.0,2.7 \mathrm{~Hz}, 1 \mathrm{H}), 4.05(\mathrm{dd}, J=14.7,4.5 \mathrm{~Hz}, 1 \mathrm{H})$, $3.33(\mathrm{dd}, J=14.7,11.4 \mathrm{~Hz}, 1 \mathrm{H}), 2.98$ (ddd, $J=10.1,2.7,2.4 \mathrm{~Hz}, 1 \mathrm{H}), 2.59$ (ddd, $J=10.2,2.4,2.1$ $\mathrm{Hz}, 1 \mathrm{H}), 2.43(\mathrm{~s}, 3 \mathrm{H}), 2.24-2.16(\mathrm{~m}, 3 \mathrm{H}), 2.16(\mathrm{dd}, J=11.0,10.1 \mathrm{~Hz}, 1 \mathrm{H}), 0.94-0.77(\mathrm{~m}, 1 \mathrm{H}) ;{ }^{13} \mathrm{C}$ $\operatorname{NMR}\left(75 \mathrm{MHz}, \mathrm{CDCl}_{3}\right): \delta 143.5,135.5,130.0,129.8,128.6,127.1,60.3,55.2,51.7,44.6,27.5,21.9$, 21.7; IR (neat, $\mathrm{cm}^{-1}$ ): 2942, 2869, 1932, 1656, 1596, 1481, 1449, 1397, 1263, 1237, 1211, 1048, 1026, 984, 708; Mp: $132{ }^{\circ} \mathrm{C}$; Analytical HPLC [column: CHIRALCEL OD-H (4.6 mm $\left.\times 25 \mathrm{~cm}\right)$, eluent: hexane $/ i-\mathrm{PrOH}=2 / 1$, flow rate: $0.5 \mathrm{~mL} / \mathrm{min}$, detection: UV $236 \mathrm{~nm}$, temperature: $\mathrm{rt}] t_{R}=$ $18.6 \mathrm{~min}$ for $(-)-(3 R, 4 R)$ - isomer, $22.7 \mathrm{~min}$ for $(+)-(3 S, 4 S)$-isomer; Optical rotation value: $[\alpha]_{\mathrm{D}}{ }^{21}$ -53.3 (c 1.16, $\left.\mathrm{CHCl}_{3}\right)$ for $(3 R, 4 R)$-isomer ( $>98 \%$ ee); HRMS (EI, positive): $\mathrm{m} / \mathrm{z}$ calcd for $\mathrm{C}_{15} \mathrm{H}_{19} \mathrm{NO}_{3} \mathrm{~S}[\mathrm{M}]^{+}:$293.1086, found: 293.1085; Anal. calcd for $\mathrm{C}_{15} \mathrm{H}_{19} \mathrm{NO}_{3} \mathrm{~S}: \mathrm{C}, 61.41 ; \mathrm{H}, 6.53$; , 4.77. Found: C, 61.33; H, 6.30; N, 4.77 .

\section{7-(p-toluenesulfonyl)-10-oxa-7-azabicyclo[7.1.0]dec-4-ene [(7R,8S)-3b]}

${ }^{1} \mathrm{H}$ NMR $\left(300 \mathrm{MHz}, \mathrm{CDCl}_{3}\right): \delta 7.69(\mathrm{~d}, J=7.8 \mathrm{~Hz}, 2 \mathrm{H}), 7.32(\mathrm{~d}, J=7.8 \mathrm{~Hz}, 2 \mathrm{H}), 5.46$ (ddd, $J=15.6$, $10.2,4.8 \mathrm{~Hz}, 1 \mathrm{H}), 5.30$ (ddd, $J=15.6,10.5,4.2 \mathrm{~Hz}, 1 \mathrm{H}), 4.51$ (dd, $J=10.8,4.2 \mathrm{~Hz}, 1 \mathrm{H}), 3.91$ (dd, $J$ $=14.7,2.1 \mathrm{~Hz}, 1 \mathrm{H}), 3.05(\mathrm{ddd}, J=9.9,3.9,2.1 \mathrm{~Hz}, 1 \mathrm{H}), 2.99(\mathrm{dd}, J=10.8,10.5 \mathrm{~Hz}, 1 \mathrm{H}), 2.96-2.90$ $(\mathrm{m}, 1 \mathrm{H}), 2.44(\mathrm{~s}, 3 \mathrm{H}), 2.42-2.24(\mathrm{~m}, 2 \mathrm{H}), 2.18(\mathrm{dd}, J=14.7,9.9 \mathrm{~Hz}, 1 \mathrm{H}), 2.12-1.99(\mathrm{~m}, 1 \mathrm{H})$, 0.89-0.76 (m, 1H); ${ }^{13} \mathrm{C}$ NMR (75 MHz, $\left.\mathrm{CDCl}_{3}\right): \delta 143.5,137.8,135.7,129.8,127.1,125.3,57.8$, 56.7, 52.1, 43.8, 28.2, 27.9, 21.7; IR (neat, $\mathrm{cm}^{-1}$ ): 2923, 2852, 1596, 1453, 1337, 1165, 1098, 983, 916, 751, 658, 584, 548; Mp: $123{ }^{\circ} \mathrm{C}$; Analytical HPLC [column: CHIRALCEL OD-H (4.6 mm $\times 25$ $\mathrm{cm}$ ), eluent: hexane $/ i-\mathrm{PrOH}=2 / 1$, flow rate: $0.5 \mathrm{~mL} / \mathrm{min}$, detection: UV $236 \mathrm{~nm}$, temperature: $\mathrm{rt}] t_{R}$ 
$=21.9 \mathrm{~min}$ for $(-)-(7 S, 8 R)$ - isomer, $34.5 \mathrm{~min}$ for $(+)-(7 R, 8 S)$-isomer; Optical rotation value: $[\alpha]_{\mathrm{D}}{ }^{20}$ 109.4 ( c 0.49, $\left.\mathrm{CHCl}_{3}\right)$ for $(7 R, 8 S)$-isomer ( $>98 \%$ ee); HRMS (FAB, matrix: 3-nitrobenzyl alcohol, positive): $\mathrm{m} / z$ calcd for $\mathrm{C}_{15} \mathrm{H}_{20} \mathrm{NO}_{3} \mathrm{~S}[\mathrm{M}+\mathrm{H}]^{+}:$294.1164, found: 294.1165; Anal. calcd for $\mathrm{C}_{15} \mathrm{H}_{19} \mathrm{NO}_{3} \mathrm{~S}$ : C, 61.41; H, 6.53; N, 4.77. Found: C, 61.74; H, 6.64; N, 4.52.

\section{8-(p-toluenesulfonyl)-5,11-dioxa-8-azatricyclo $\left[8.1 .0 .0^{4,6}\right]$ undecane $[(3 R, 4 R, 7 R, 8 S)-4 b]$}

${ }^{1} \mathrm{H}$ NMR $\left(300 \mathrm{MHz}, \mathrm{CDCl}_{3}\right): \delta 7.68(\mathrm{~d}, J=8.3 \mathrm{~Hz}, 2 \mathrm{H}), 7.32(\mathrm{~d}, J=8.3 \mathrm{~Hz}, 2 \mathrm{H}), 4.43(\mathrm{dd}, J=11.1$, $2.6 \mathrm{~Hz}, 1 \mathrm{H}), 4.10(\mathrm{~d}, J=15.0 \mathrm{~Hz}, 1 \mathrm{H}), 3.23-3.18(\mathrm{~m}, 1 \mathrm{H}), 3.05-2.99(\mathrm{~m}, 1 \mathrm{H}), 2.83$ (ddd, $J=9.9,2.6$, $2.4 \mathrm{~Hz}, 1 \mathrm{H}), 2.51-2.39(\mathrm{~m}, 2 \mathrm{H}), 2.47(\mathrm{dd}, J=15.0,10.0 \mathrm{~Hz}, 1 \mathrm{H}), 2.44(\mathrm{~s}, 3 \mathrm{H}), 2.32-2.25(\mathrm{~m}, 1 \mathrm{H})$, $2.10(\mathrm{dd}, J=11.1,9.9 \mathrm{~Hz}, 1 \mathrm{H}), 1.26-1.10(\mathrm{~m}, 2 \mathrm{H}) ;{ }^{13} \mathrm{C} \mathrm{NMR}\left(75 \mathrm{MHz}, \mathrm{CDCl}_{3}\right): \delta 143.9,135.1$, 130.0, 127.1, 60.0, 57.1, 57.0, 55.0, 51.2, 44.3, 26.5, 24.4, 21.7; IR (neat, $\mathrm{cm}^{-1}$ ): 2931, 2870, 1597 , 1492, 1474, 1450, 1301, 1264, 1239, 1215, 1181, 1160, 1073, 992, 911, 811, 600; Mp: $169{ }^{\circ} \mathrm{C}$; Analytical HPLC [column: CHIRALCEL OD-H $(4.6 \mathrm{~mm} \times 25 \mathrm{~cm})$, eluent: hexane $/ i-\mathrm{PrOH}=6 / 1$, flow rate: $0.5 \mathrm{~mL} / \mathrm{min}$, detection: UV $254 \mathrm{~nm}$, temperature: $\mathrm{rt}] t_{R}=43.5 \mathrm{~min}$ for $(+)-(3 S, 4 S, 7 S, 8 R)$-isomer, $45.6 \mathrm{~min}$ for $(-)-(3 R, 4 R, 7 R, 8 S)$-isomer; Optical rotation value: $[\alpha]_{\mathrm{D}}{ }^{27}$ -21.6 ( $\left.c 0.37, \mathrm{CHCl}_{3}\right)$ for $(3 R, 4 R, 7 R, 8 S)$ - isomer ( $>98 \%$ ee); HRMS (EI, positive): $\mathrm{m} / z$ calcd for $\mathrm{C}_{15} \mathrm{H}_{19} \mathrm{NO}_{4} \mathrm{~S}[\mathrm{M}]^{+}:$309.1035, found: 309.1036; Anal. calcd for $\mathrm{C}_{15} \mathrm{H}_{19} \mathrm{NO}_{4} \mathrm{~S}: \mathrm{C}, 58.23 ; \mathrm{H}, 6.19$; N, 4.53. Found: C, 57.97; H, 5.98; N, 4.47 .

\section{3) Cyclopropanation reaction of cyclic amide 1a}

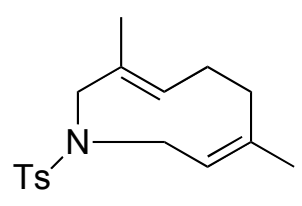

$(R)-1 \mathbf{a}$ $(>98 \%$ ee $)$

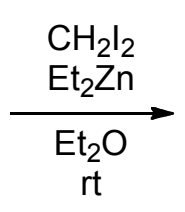

$(3 R, 4 R)-5 a$ $(>98 \%$ dr, $>98 \%$ ee)

$69 \%$

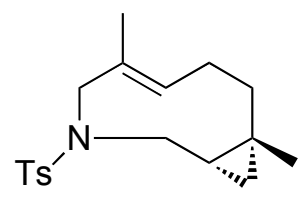

$(7 R, 8 S)-6 \mathbf{a}$ $(>98 \% \mathrm{dr},>98 \%$ ee) $2 \%$

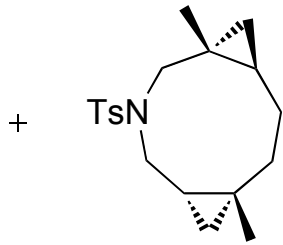

$(3 R, 4 R, 7 R, 8 S)-7 \mathbf{a}$ $(>98 \% \mathrm{dr},>98 \%$ ee $)$

$16 \%$

To a solution of $\mathrm{Et}_{2} \mathrm{Zn}(1.10 \mathrm{M}$ solution in hexane, $0.595 \mathrm{~mL}, 0.655 \mathrm{mmol})$ in $\operatorname{dry} \mathrm{Et}_{2} \mathrm{O}(10 \mathrm{~mL})$ at room temperature was slowly added diiodomethane $(53.7 \mu \mathrm{L}, 0.668 \mathrm{mmol})$. After stirring at that temperature for $15 \mathrm{~min},(R)-\mathbf{1 a}(>98 \%$ ee; $40.0 \mathrm{mg}, 0.131 \mathrm{mmol})$ was added to the mixture. The mixture was stirred at room temperature for $24 \mathrm{~h}$. The reaction was quenched with saturated aqueous $\mathrm{NH}_{4} \mathrm{Cl}$. The phases were separated and the aqueous layer was extracted with AcOEt. The combined organic phases were dried over $\mathrm{Na}_{2} \mathrm{SO}_{4}$, filtered and the solvent was removed in vacuo. The residue was purified by silica gel chromatography (hexane/AcOEt $=1 / 0$ to $12 / 1$ ) to afford $37.0 \mathrm{mg}$ of a mixture of 5a, 6a and 7a $\left(87 \% ; \mathbf{5 a} / \mathbf{6 a} / 7 \mathbf{a}=79 / 2 / 19\right.$ determined by ${ }^{1} \mathrm{H}$ NMR analysis $)$ as a white solid. Di-cyclopropanated product $7 \mathbf{a}$ was separated from a mixture of mono-cyclopropanated products 5a and 6a by silica gel HPLC; column: Kanto Mightysil Si $60(5 \mu \mathrm{m})(2.0 \mathrm{~cm} \times 25 \mathrm{~cm})$, eluent: hexane/AcOEt = 9/1, flow rate: $7.0 \mathrm{~mL} / \mathrm{min}$, detection: UV $254 \mathrm{~nm}$, temperature: rt. Then the mixture of 5a and 6a were separated by chiral HPLC; column: Daicel CHIRALCEL OD-H column $(2.0 \mathrm{~cm} \times 25 \mathrm{~cm})$, eluent: hexane $/ i-\mathrm{PrOH}=4 / 1$, flow rate: $5.0 \mathrm{~mL} / \mathrm{min}$, detection: UV $254 \mathrm{~nm}$, temperature: $\mathrm{rt}$. All of cyclopropanated products $(3 R, 4 R)-\mathbf{5 a},(7 R, 8 S)-\mathbf{6 a}$ and $(3 R, 4 R, 7 R, 8 S)-7 \mathbf{a}$ were determined as a single stereoisomer $\left(>98 \% \mathrm{dr}\right.$ analyzed by ${ }^{1} \mathrm{H}$ NMR, $>98 \%$ ee analyzed by chiral HPLC). 


\section{4,9-dimethyl-7-(p-toluenesulfonyl)-7-azabicyclo[7.1.0]dec-4-ene [(3R,4R)-5a]}

${ }^{1} \mathrm{H}$ NMR (300 MHz, $\mathrm{CDCl}_{3}$ ): $\delta 7.63(\mathrm{~d}, J=7.8 \mathrm{~Hz}, 2 \mathrm{H}), 7.27$ (d, $\left.J=7.8 \mathrm{~Hz}, 2 \mathrm{H}\right), 5.49$ (dd, $J=11.6$, $4.4 \mathrm{~Hz}, 1 \mathrm{H}), 4.10(\mathrm{~d}, J=11.7 \mathrm{~Hz}, 1 \mathrm{H}), 3.96(\mathrm{dd}, J=14.3,4.4 \mathrm{~Hz}, 1 \mathrm{H}), 3.44(\mathrm{dd}, J=14.3,11.6 \mathrm{~Hz}$, $1 \mathrm{H}), 2.41(\mathrm{~s}, 3 \mathrm{H}), 2.41-2.31(\mathrm{~m}, 1 \mathrm{H}), 1.97-1.81(\mathrm{~m}, 3 \mathrm{H}), 1.72(\mathrm{~s}, 3 \mathrm{H}), 0.97(\mathrm{~s}, 3 \mathrm{H}), 0.92-0.76(\mathrm{~m}$, $1 \mathrm{H}), 0.59-0.49(\mathrm{~m}, 1 \mathrm{H}), 0.36(\mathrm{dd}, J=9.3,4.8 \mathrm{~Hz}, 1 \mathrm{H}),-0.01(\mathrm{dd}, J=6.3,4.8 \mathrm{~Hz}, 1 \mathrm{H}) ;{ }^{13} \mathrm{C} \mathrm{NMR}$ $\left(75 \mathrm{MHz}, \mathrm{CDCl}_{3}\right): \delta 142.7,136.7,135.6,129.6,126.9,123.9,59.3,45.6,32.8,28.6,25.4,23.3,21.6$, 17.9, 17.5, 13.8; IR (neat, $\mathrm{cm}^{-1}$ ): 2922, 1457, 1317, 1152, 1091, 1050, 995, 946, 894, 844, 812, 752, 655; Mp: $105{ }^{\circ} \mathrm{C}$; Analytical HPLC [column: CHIRALCEL OD-H (4.6 mm $\left.\times 25 \mathrm{~cm}\right)$, eluent: hexane $/ i-\mathrm{PrOH}=4 / 1$, flow rate: $0.5 \mathrm{~mL} / \mathrm{min}$, detection: UV $254 \mathrm{~nm}$, temperature: $\mathrm{rt}] t_{R}=12.0 \mathrm{~min}$ for $(-)-(3 S, 4 S)$-isomer, $22.7 \mathrm{~min}$ for $(+)-(3 R, 4 R)$-isomer; Optical rotation value: $[\alpha]_{\mathrm{D}}{ }^{23} 55.8(c 1.14$, $\mathrm{CHCl}_{3}$ ) for $(3 R, 4 R)$-isomer ( $>98 \%$ ee); HRMS (EI, positive): $m / z$ calcd for $\mathrm{C}_{18} \mathrm{H}_{25} \mathrm{NO}_{2} \mathrm{~S}[\mathrm{M}]^{+}$: 319.1606, found: 319.1612; Anal. calcd for $\mathrm{C}_{18} \mathrm{H}_{25} \mathrm{NO}_{2} \mathrm{~S}$ : C, 67.67; H, 7.89; N, 4.38. Found: C, $67.58 ; \mathrm{H}, 7.85 ; \mathrm{N}, 4.34$.

\section{5,9-dimethyl-3-(p-toluenesulfonyl)-3-azabicyclo[7.1.0]dec-5-ene [(7R,8S)-6a]}

${ }^{1} \mathrm{H}$ NMR $\left(300 \mathrm{MHz}, \mathrm{CDCl}_{3}\right): \delta 7.66(\mathrm{~d}, J=8.1 \mathrm{~Hz}, 2 \mathrm{H}), 7.29(\mathrm{~d}, J=8.1 \mathrm{~Hz}, 2 \mathrm{H}), 5.15(\mathrm{dd}, J=11.4$, $3.0 \mathrm{~Hz}, 1 \mathrm{H}), 4.35(\mathrm{~d}, J=10.4 \mathrm{~Hz}, 1 \mathrm{H}), 3.76(\mathrm{~d}, J=14.9 \mathrm{~Hz}, 1 \mathrm{H}), 2.96(\mathrm{~d}, J=10.4 \mathrm{~Hz}, 1 \mathrm{H})$, 2.50-2.36 (m, 1H), $2.43(\mathrm{~s}, 3 \mathrm{H}), 2.18(\mathrm{dd}, J=14.9,9.9 \mathrm{~Hz}, 1 \mathrm{H}), 2.04-1.98(\mathrm{~m}, 2 \mathrm{H}), 1.81(\mathrm{~s}, 3 \mathrm{H})$, $1.04(\mathrm{~s}, 3 \mathrm{H}), 0.93-0.71(\mathrm{~m}, 2 \mathrm{H}), 0.45(\mathrm{dd}, J=9.6,4.5 \mathrm{~Hz}, 1 \mathrm{H}),-0.27(\mathrm{dd}, J=6.6,4.5 \mathrm{~Hz}, 1 \mathrm{H}) ;{ }^{13} \mathrm{C}$ $\operatorname{NMR}\left(75 \mathrm{MHz}, \mathrm{CDCl}_{3}\right): \delta 142.8,136.7,132.6,129.6,128.5,126.9,58.8,46.9,34.9,28.3,26.0,25.0$, 21.7, 20.4, 16.9, 16.0; IR (neat, $\mathrm{cm}^{-1}$ ): 2931, 1452, 1327, 1156, 1087, 1024, 922, 881, 809, 756, 660, 592, 546; Mp: $104{ }^{\circ} \mathrm{C}$; Analytical HPLC [column: CHIRALCEL OD-H (4.6 mm $\times 25 \mathrm{~cm}$ ), eluent: hexane $/ i-\mathrm{PrOH}=4 / 1$, flow rate: $0.5 \mathrm{~mL} / \mathrm{min}$, detection: UV $254 \mathrm{~nm}$, temperature: $\mathrm{rt}] t_{R}=12.0 \mathrm{~min}$ for $(-)-(7 S, 8 R)$-isomer, $35.2 \mathrm{~min}$ for $(+)-(7 R, 8 S)$-isomer; Optical rotation value: $[\alpha]_{\mathrm{D}}{ }^{24} 69.8(c 0.16$, $\mathrm{CHCl}_{3}$ ) for $(7 R, 8 S)$-isomer ( $>98 \%$ ee); HRMS (EI, positive): $m / z$ calcd for $\mathrm{C}_{18} \mathrm{H}_{25} \mathrm{NO}_{2} \mathrm{~S}[\mathrm{M}]^{+}$: 319.1606, found: 319.1614 .

\section{1,6-dimethyl-8-(p-toluenesulfonyl)-8-azatricyclo $\left[8.1 .0 .0^{4,6}\right]$ undecane $[(3 R, 4 R, 7 R, 8 S)-7 \mathrm{a}]$}

${ }^{1} \mathrm{H}$ NMR $\left(300 \mathrm{MHz}, \mathrm{CDCl}_{3}\right): \delta 7.65(\mathrm{~d}, J=7.8 \mathrm{~Hz}, 2 \mathrm{H}), 7.27(\mathrm{~d}, J=7.8 \mathrm{~Hz}, 2 \mathrm{H}), 4.19$ (d, $J=12.0$ $\mathrm{Hz}, 1 \mathrm{H}), 3.85$ (d, $J=15.3 \mathrm{~Hz}, 1 \mathrm{H}), 2.47(\mathrm{dd}, J=15.3,9.9 \mathrm{~Hz}, 1 \mathrm{H}), 2.41$ (s, 3H), 2.08 (dd, $J=14.4$, $5.7 \mathrm{~Hz}, 1 \mathrm{H}), 1.82$ (dd, $J=14.1,5.7 \mathrm{~Hz}, 1 \mathrm{H}), 1.76(\mathrm{~d}, J=12.0 \mathrm{~Hz}, 1 \mathrm{H}), 1.27-1.14(\mathrm{~m}, 2 \mathrm{H}), 1.24$ (s, $3 \mathrm{H}), 1.11-1.01(\mathrm{~m}, 1 \mathrm{H}), 1.05(\mathrm{~s}, 3 \mathrm{H}), 0.50(\mathrm{dd}, J=9.8,4.5 \mathrm{~Hz}, 1 \mathrm{H}), 0.39-0.28(\mathrm{~m}, 2 \mathrm{H}), 0.09-0.03(\mathrm{~m}$, $1 \mathrm{H}),-0.16(\mathrm{dd}, J=6.3,4.5 \mathrm{~Hz}, 1 \mathrm{H}) ;{ }^{13} \mathrm{C} \mathrm{NMR}\left(75 \mathrm{MHz}, \mathrm{CDCl}_{3}\right): \delta 142.7,136.7,129.6,126.8,60.1$, 47.8, 37.0, 28.8, 28.5, 24.8, 24.5, 21.7, 21.5, 18.1, 17.8, 17.7, 13.3; IR (neat, $\mathrm{cm}^{-1}$ ): 2909, 1456, 1341, 1329, 1302, 1157, 1088, 1037, 939, 810, 747, 657; Mp: $115^{\circ} \mathrm{C}$; Analytical HPLC [column: CHIRALCEL OD-H $(4.6 \mathrm{~mm} \times 25 \mathrm{~cm})$, eluent: hexane $/ i$-PrOH $=4 / 1$, flow rate: $0.5 \mathrm{~mL} / \mathrm{min}$, detection: UV $254 \mathrm{~nm}$, temperature: $\mathrm{rt}] t_{R}=11.2 \mathrm{~min}$ for $(-f)-(3 S, 4 S, 7 S, 8 R)$-isomer, 11.6 min for $(+)-(3 R, 4 R, 7 R, 8 S)$-isomer; Optical rotation value: $[\alpha]_{\mathrm{D}}{ }^{24} 18.1 \quad\left(c \quad 0.55, \mathrm{CHCl}_{3}\right)$ for (3R,4R,7R,8S)-isomer ( $>98 \%$ ee); HRMS (FAB, matrix: 3-nitrobenzyl alcohol, positive): $\mathrm{m} / \mathrm{z}$ calcd for $\mathrm{C}_{19} \mathrm{H}_{28} \mathrm{NO}_{2} \mathrm{~S}[\mathrm{M}+\mathrm{H}]^{+}: 334.1841$, found: 334.1843; Anal. calcd for $\mathrm{C}_{19} \mathrm{H}_{27} \mathrm{NO}_{2} \mathrm{~S}$ : C, 68.43; $\mathrm{H}$, 8.16; N, 4.20. Found: C, 68.14; H, 8.05; N, 4.15. 


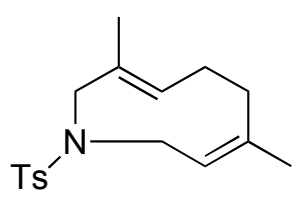

$(R)-1 a$

$(>98 \%$ ee)

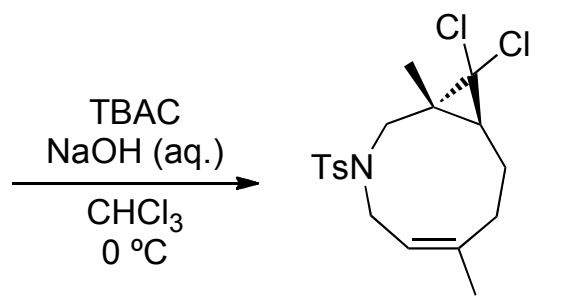

$(3 R, 4 S)-5 \mathbf{b}$ $(>98 \% \mathrm{dr},>98 \%$ ee $)$

$44 \%$

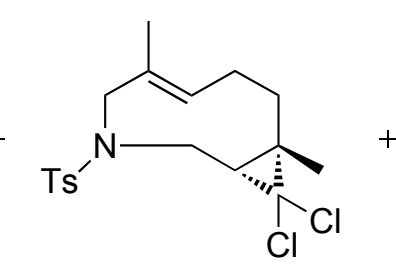

$(7 S, 8 S)-6 \mathbf{b}$ $(>98 \%$ dr, $>98 \%$ ee $)$ $14 \%$

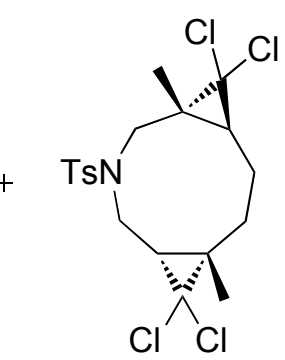

$(3 R, 4 S, 7 S, 8 S)-7 \mathbf{b}$ $(>98 \% \mathrm{dr},>98 \%$ ee)

$36 \%$

To a solution of $(R)-1 \mathrm{a}(>98 \%$ ee; $77.8 \mathrm{mg}, 0.255 \mathrm{mmol})$ in dry $\mathrm{CHCl}_{3}(6 \mathrm{~mL})$ at $0{ }^{\circ} \mathrm{C}$ was added $\mathrm{NaOH}(1.02 \mathrm{~g}, 25.5 \mathrm{mmol})$ dissolved in water $(1.5 \mathrm{~mL})$ and tetrabutylammonium chloride $(14.2 \mathrm{mg}$, $0.0509 \mathrm{mmol})$. The mixture was stirred vigorously at that temperature for $20 \mathrm{~h}$. The reaction was quenched by diluting with water. The phases were separated and the aqueous layer was extracted with $\mathrm{CH}_{2} \mathrm{Cl}_{2}$. The combined organic phases were dried over $\mathrm{Na}_{2} \mathrm{SO}_{4}$, filtered and the solvent was removed in vacuo. The residue was purified by silica gel chromatography (hexane $/ \mathrm{CH}_{2} \mathrm{Cl}_{2}=10 / 1$ to $1 / 1)$ to afford $57.1 \mathrm{mg}$ of a mixture of mono-cyclopropanated products $\mathbf{5 b}$ and $\mathbf{6 b}(58 \% ; \mathbf{5 b} / \mathbf{6 b}=$ $76 / 24$ determined by ${ }^{1} \mathrm{H}$ NMR analysis) as a white solid and $43.3 \mathrm{mg}(36 \%)$ of $\mathbf{7 b}$ as a white solid. The mixture of $\mathbf{5 b}$ and $\mathbf{6 b}$ were separated by chiral HPLC; column: Daicel CHIRALPAK AS-H column $(2.0 \mathrm{~cm} \times 25 \mathrm{~cm})$, eluent: hexane $/ i-\mathrm{PrOH}=2 / 1$, flow rate: $3.0 \mathrm{~mL} / \mathrm{min}$, detection: UV 254 $\mathrm{nm}$, temperature: rt. All of cyclopropanated products $(3 R, 4 S)-\mathbf{5 b},(7 S, 8 S)-\mathbf{6 b}$ and $(3 R, 4 S, 7 S, 8 S)-7 \mathbf{b}$ were determined as a single stereoisomer $\left(>98 \% \mathrm{dr}\right.$ analyzed by ${ }^{1} \mathrm{H} \mathrm{NMR},>98 \%$ ee analyzed by chiral HPLC).

\section{0,10-dichloro-4,9-dimethyl-7-(p-toluenesulfonyl)-7-azabicyclo[7.1.0]dec-4-ene [(3R,4S)-5b]}

${ }^{1} \mathrm{H}$ NMR $\left(300 \mathrm{MHz}, \mathrm{CDCl}_{3}\right): \delta 7.65(\mathrm{~d}, J=8.4 \mathrm{~Hz}, 2 \mathrm{H}), 7.30(\mathrm{~d}, J=8.4 \mathrm{~Hz}, 2 \mathrm{H}), 5.51(\mathrm{dd}, J=10.8$, $3.9 \mathrm{~Hz}, 1 \mathrm{H}), 4.16$ (br, 1H), 3.96 (br, 1H), 3.23 (br, 1H), 2.50-2.30 (m, 2H), 2.43 (s, 3H), 2.05-1.98 $(\mathrm{m}, 2 \mathrm{H}), 1.74(\mathrm{~s}, 3 \mathrm{H}), 1.40-1.32(\mathrm{~m}, 1 \mathrm{H}), 1.16(\mathrm{~s}, 3 \mathrm{H}), 1.08-0.97(\mathrm{~m}, 1 \mathrm{H}) ;{ }^{13} \mathrm{C} \mathrm{NMR}(75 \mathrm{MHz}$, $\left.\mathrm{CDCl}_{3}\right): \delta 143.2,136.3,135.1,129.8,126.9,123.9,67.3,56.5,45.5,42.5,30.8,29.9,25.2,21.7$, 20.2, 15.0; IR (neat, $\mathrm{cm}^{-1}$ ): 2928, 1598, 1457, 1334, 1160, 1092, 1045, 934, 907, 862, 846, 798, 765, 661; Mp: $106{ }^{\circ} \mathrm{C}$; Analytical HPLC [column: CHIRALCEL OD-H $(4.6 \mathrm{~mm} \times 25 \mathrm{~cm})$, eluent: hexane $/ \mathrm{EtOH}=10 / 1$, flow rate: $0.5 \mathrm{~mL} / \mathrm{min}$, detection: $\mathrm{UV} 254 \mathrm{~nm}$, temperature: $\mathrm{rt}] t_{R}=14.2 \mathrm{~min}$ for $(-)-(3 S, 4 R)$-isomer, $31.9 \mathrm{~min}$ for $(+)-(3 R, 4 S)$-isomer; Optical rotation value: $[\alpha]_{\mathrm{D}}{ }^{24} 94.1(c 1.40$, $\mathrm{CHCl}_{3}$ ) for $(3 R, 4 S)$-isomer ( $>98 \%$ ee); HRMS (EI, positive): $m / z$ calcd for $\mathrm{C}_{18} \mathrm{H}_{23} \mathrm{Cl}_{2} \mathrm{NO}_{2} \mathrm{~S}[\mathrm{M}]^{+}$: 387.0827, found: 387.0819; Anal. calcd for $\mathrm{C}_{18} \mathrm{H}_{23} \mathrm{Cl}_{2} \mathrm{NO}_{2} \mathrm{~S} \cdot 1 / 8\left(\mathrm{C}_{6} \mathrm{H}_{14}\right)$ : C, 56.42; $\mathrm{H}, 6.25 ; \mathrm{N}, 3.51$. Found: C, 56.47; H, 6.22; N, 3.50.

\section{0,10-dichloro-5,9-dimethyl-3-(p-toluenesulfonyl)-3-azabicyclo[7.1.0]dec-5-ene [(7S,8S)-6b]}

${ }^{1} \mathrm{H}$ NMR $\left(300 \mathrm{MHz}, \mathrm{CDCl}_{3}\right): \delta 7.69(\mathrm{~d}, J=8.3 \mathrm{~Hz}, 2 \mathrm{H}), 7.32(\mathrm{~d}, J=8.3 \mathrm{~Hz}, 2 \mathrm{H}), 5.15(\mathrm{dd}, J=11.7$, $3.6 \mathrm{~Hz}, 1 \mathrm{H}), 4.33(\mathrm{~d}, J=10.5 \mathrm{~Hz}, 1 \mathrm{H}), 3.86(\mathrm{~d}, J=15.2 \mathrm{~Hz}, 1 \mathrm{H}), 2.95(\mathrm{~d}, J=10.5 \mathrm{~Hz}, 1 \mathrm{H})$, 2.57-2.44 (m, 1H), $2.44(\mathrm{~s}, 3 \mathrm{H}), 2.31(\mathrm{dd}, J=15.2,9.3 \mathrm{~Hz}, 1 \mathrm{H}), 2.22-2.14(\mathrm{~m}, 1 \mathrm{H}), 1.93-1.81(\mathrm{~m}$, 1H), 1.77 (s, 3H), 2.59-1.39 (m, 2H), $1.37(\mathrm{~s}, 3 \mathrm{H}) ;{ }^{13} \mathrm{C} \mathrm{NMR}\left(75 \mathrm{MHz}, \mathrm{CDCl}_{3}\right): \delta 143.2,136.3$, 131.7, 129.8, 129.1, 126.9, 69.5, 58.9, 44.7, 41.0, 31.9, 30.3, 25.9, 23.5, 21.7, 15.9; IR (neat, $\mathrm{cm}^{-1}$ ): 2932, 1456, 1347, 1161, 1096, 1009, 921, 886, 840, 756, 662, 592; Mp: $167{ }^{\circ} \mathrm{C}$; Analytical HPLC [column: CHIRALCEL OD-H $(4.6 \mathrm{~mm} \times 25 \mathrm{~cm})$, eluent: hexane/EtOH $=10 / 1$, flow rate: 0.5 $\mathrm{mL} / \mathrm{min}$, detection: UV $254 \mathrm{~nm}$, temperature: $\mathrm{rt}] t_{R}=12.6 \mathrm{~min}$ for $(+)-(7 S, 8 S)$-isomer, $34.2 \mathrm{~min}$ for 
$(-)-(7 R, 8 R)$-isomer; Optical rotation value: $[\alpha]_{\mathrm{D}}{ }^{24} 130.6\left(c 0.24, \mathrm{CHCl}_{3}\right)$ for $(7 S, 8 S)$-isomer $(>98 \%$ ee); HRMS (FAB, matrix: 3-nitrobenzyl alcohol, positive): $m / z$ calcd for $\mathrm{C}_{18} \mathrm{H}_{24} \mathrm{Cl}_{2} \mathrm{NO}_{2} \mathrm{~S}[\mathrm{M}+\mathrm{H}]^{+}$: 388.0905, found: 388.0901; Anal. calcd for $\mathrm{C}_{18} \mathrm{H}_{23} \mathrm{Cl}_{2} \mathrm{NO}_{2} \mathrm{~S}$ : C, 55.67; H, 5.97; N, 3.61. Found: C, $55.66 ; \mathrm{H}, 5.98 ; \mathrm{N}, 3.57$.

\section{$5,5,11,11-$ tetrachloro-1,6-dimethyl-8-( $p$-toluenesulfonyl)-8-azatricyclo[8.1.0.0 $\left.{ }^{4,6}\right]$ undecane $[(3 R, 4 S, 7 S, 8 S)-7 \mathrm{~b}]$}

${ }^{1} \mathrm{H}$ NMR $\left(300 \mathrm{MHz}, \mathrm{CDCl}_{3}\right): \delta 7.70(\mathrm{~d}, J=8.3 \mathrm{~Hz}, 2 \mathrm{H}), 7.34(\mathrm{~d}, J=8.3 \mathrm{~Hz}, 2 \mathrm{H}), 4.26(\mathrm{~d}, J=12.5$ $\mathrm{Hz}, 1 \mathrm{H}), 3.95(\mathrm{~d}, J=15.8 \mathrm{~Hz}, 1 \mathrm{H}), 2.44(\mathrm{~s}, 3 \mathrm{H}), 2.40(\mathrm{~d}, J=12.5 \mathrm{~Hz}, 1 \mathrm{H}), 2.38(\mathrm{dd}, J=15.8,9.3 \mathrm{~Hz}$, $1 \mathrm{H}), 2.19-2.12(\mathrm{~m}, 1 \mathrm{H}), 2.08-2.01(\mathrm{~m}, 1 \mathrm{H}), 1.85(\mathrm{dd}, J=9.3,1.2 \mathrm{~Hz}, 1 \mathrm{H}), 1.77(\mathrm{~d}, J=13.5 \mathrm{~Hz}, 1 \mathrm{H})$, 1.48-1.37 (m, 1H), $1.37(\mathrm{~s}, 6 \mathrm{H}), 1.17(\mathrm{dd}, J=11.1,1.8 \mathrm{~Hz}, 1 \mathrm{H}) ;{ }^{13} \mathrm{C}$ NMR $\left(75 \mathrm{MHz}, \mathrm{CDCl}_{3}\right): \delta$ $143.7,135.8,130.0,126.9,69.0,66.3,57.3,45.8,41.5,40.5,32.1,30.7,30.1,22.9,21.72,21.69$, 14.5; IR (neat, $\mathrm{cm}^{-1}$ ): 2989, 2963, 2921, 2862, 1599, 1454, 1321, 1155, 930, 766, 666, 551, 531; Mp: $166{ }^{\circ} \mathrm{C}$; Analytical HPLC [column: CHIRALCEL OD-H (4.6 mm $\left.\times 25 \mathrm{~cm}\right)$, eluent: hexane $/ \mathrm{EtOH}=30 / 1$, flow rate: $0.5 \mathrm{~mL} / \mathrm{min}$, detection: $\mathrm{UV} 254 \mathrm{~nm}$, temperature: $\mathrm{rt}] t_{R}=15.5 \mathrm{~min}$ for $(-)-(3 S, 4 R, 7 R, 8 R)$-isomer, $16.8 \mathrm{~min}$ for $(+)-(3 R, 4 S, 7 S, 8 S)$ - isomer; Optical rotation value: $[\alpha]_{\mathrm{D}}{ }^{28}$ 6.27 (c 1.38, $\left.\mathrm{CHCl}_{3}\right)$ for $(3 R, 4 S, 7 S, 8 S)$-isomer ( $>98 \%$ ee); HRMS (FAB, matrix: 3-nitrobenzyl alcohol, positive): $m / z$ calcd for $\mathrm{C}_{19} \mathrm{H}_{24} \mathrm{Cl}_{4} \mathrm{NO}_{2} \mathrm{~S}[\mathrm{M}+\mathrm{H}]^{+}: 472.0252$, found: 472.0253; Anal. calcd for $\mathrm{C}_{19} \mathrm{H}_{23} \mathrm{Cl}_{4} \mathrm{NO}_{2} \mathrm{~S}$ : C, 48.42; H, 4.92; N, 2.97. Found: C, 48.35; H, 5.02; N, 2.99.

Recrystallization from hexane/ $\mathrm{CH}_{2} \mathrm{Cl}_{2}$ afforded a single crystal that was suitable for X-ray analysis.

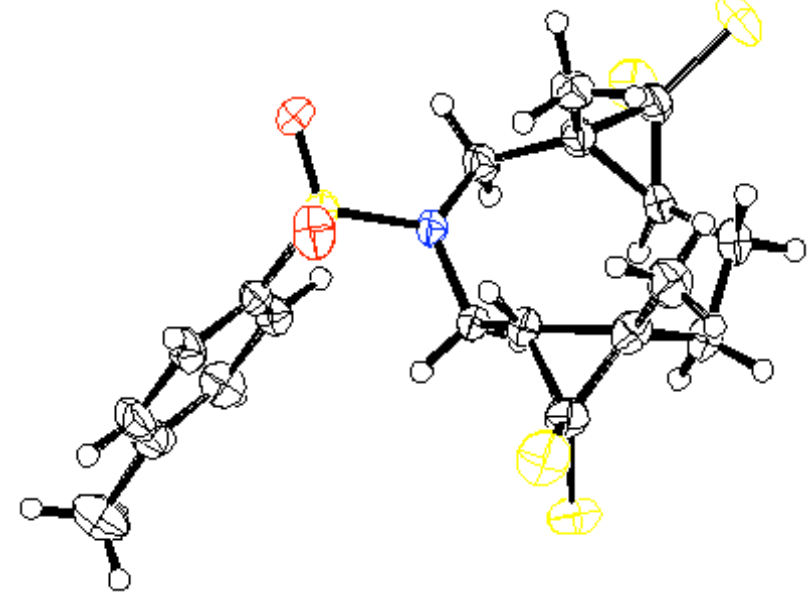

Figure S2. Molecular structure of rac-7b (CCDC-817442) in the solid state. Thermal ellipsoids are drawn at the $50 \%$ probability level. 


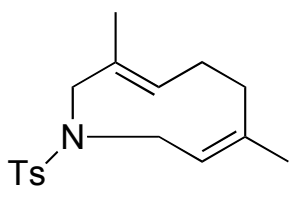

$(S)-1 a$

$(>98 \%$ ee $)$

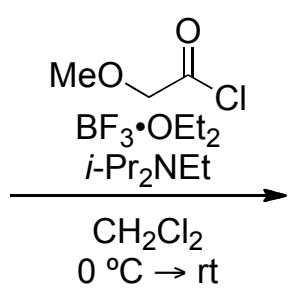

$81 \%$

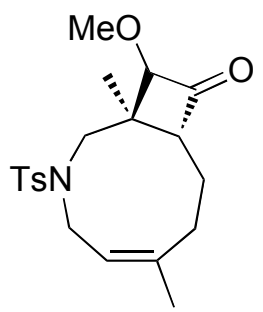

$(3 S, 4 R)-8$

(>98\% dr, >98\% ee)

To a solution of $(S)-\mathbf{1 a}(>98 \%$ ee; $31.9 \mathrm{mg}, 0.104 \mathrm{mmol})$ in dry $\mathrm{CH}_{2} \mathrm{Cl}_{2}(7 \mathrm{~mL})$ at $0{ }^{\circ} \mathrm{C}$ was added, successively, ethyldiisopropylamine $(54.6 \mu \mathrm{L}, 0.312 \mathrm{mmol})$, methoxyacetyl chloride $(19.1 \mu \mathrm{L}, 0.209$ mmol) and $\mathrm{BF}_{3} \cdot \mathrm{OEt}_{2}(26.4 \mu \mathrm{L}, 0.209 \mathrm{mmol})$. The reaction mixture was allowed to warm up to room temperature and was stirred for 3 days. The reaction was quenched with saturated aqueous $\mathrm{NaHCO}_{3}$. The phases were separated and the aqueous layer was extracted with $\mathrm{CH}_{2} \mathrm{Cl}_{2}$. The combined organic phases were dried over $\mathrm{Na}_{2} \mathrm{SO}_{4}$, filtered and the solvent was removed in vacuo. The residue was purified by silica gel chromatography (hexane/AcOEt $=30 / 1$ to 6/1) to afford $32.0 \mathrm{mg}$ of $(3 S, 4 R)-8$ $\left(81 \% ;>98 \% \mathrm{dr}\right.$ analyzed by ${ }^{1} \mathrm{H}$ NMR, $>98 \%$ ee analyzed by chiral HPLC) as a white solid and 6.1 mg of the recovered starting material $(S)-1 \mathbf{a}(19 \% ;>98 \%$ ee analyzed by HPLC).

\section{0-methoxy-4,9-dimethyl-7-(p-toluenesulfonyl)-7-azabicyclo[7.2.0]undec-4-en-11-one $[(3 S, 4 R)-8]$}

${ }^{1} \mathrm{H}$ NMR $\left(300 \mathrm{MHz}, \mathrm{CDCl}_{3}\right): \delta 7.71(\mathrm{~d}, J=8.3 \mathrm{~Hz}, 1 \mathrm{H}), 7.33(\mathrm{~d}, J=8.3 \mathrm{~Hz}, 1 \mathrm{H}), 5.53(\mathrm{dd}, J=8.6$, $8.0 \mathrm{~Hz}, 1 \mathrm{H}), 4.54$ (brs, 1H), 3.77 (dd, $J=13.8,8.6 \mathrm{~Hz}, 1 \mathrm{H}), 3.73$ (d, $J=14.7 \mathrm{~Hz}, 1 \mathrm{H}), 3.54$ (s, 3H), $3.37(\mathrm{dd}, J=13.8,8.0 \mathrm{~Hz}, 1 \mathrm{H}), 3.13(\mathrm{~d}, J=14.7 \mathrm{~Hz}, 1 \mathrm{H}), 2.95(\mathrm{~d}, J=11.7 \mathrm{~Hz}, 1 \mathrm{H}), 2.44(\mathrm{~s}, 3 \mathrm{H})$, 2.12-1.99 (m, 2H), 1.91-1.82 (m, 1H), $1.73(\mathrm{~s}, 3 \mathrm{H}), 1.48-1.37(\mathrm{~m}, 1 \mathrm{H}), 0.89(\mathrm{~s}, 3 \mathrm{H}) ;{ }^{13} \mathrm{C}$ NMR $(75$ $\left.\mathrm{MHz}, \mathrm{CDCl}_{3}\right): \delta 205.4,143.5,141.3,135.8,129.8,127.1,121.7,89.8,59.5,59.2,56.7,48.6,38.3$, 29.8, 23.6, 21.7, 18.6, 14.0; IR (reflection, $\mathrm{cm}^{-1}$ ): 2940, 1781, 1597, 1452, 1334, 1221, 1156, 1040, 811, 760, 552; Mp: $132{ }^{\circ} \mathrm{C}$; Analytical HPLC [column: CHIRALPAK AD-H (4.6 mm $\left.\times 25 \mathrm{~cm}\right)$, eluent: hexane $/ i$-PrOH $=4 / 1$, flow rate: $0.5 \mathrm{~mL} / \mathrm{min}$, detection: UV $254 \mathrm{~nm}$, temperature: $\mathrm{rt}] t_{R}=$ $33.4 \mathrm{~min}$ for $(+)-(3 R, 4 S)$-isomer, $34.4 \mathrm{~min}$ for $(-)-(3 S, 4 R)$-isomer; Optical rotation value: $[\alpha]_{\mathrm{D}}{ }^{29}$ -35.1 (c 0.76, $\mathrm{CHCl}_{3}$ ) for (3S,4R)-isomer ( $>98 \%$ ee); HRMS (FAB, matrix: 3-nitrobenzyl alcohol, positive): $\mathrm{m} / \mathrm{z}$ calcd for $\mathrm{C}_{20} \mathrm{H}_{28} \mathrm{NO}_{4} \mathrm{~S}[\mathrm{M}+\mathrm{H}]^{+}$: 378.1739, found: 378.1732; Anal. calcd for $\mathrm{C}_{20} \mathrm{H}_{27} \mathrm{NO}_{4} \mathrm{~S}$ : C, 63.63; H, 7.21; N, 3.71. Found: C, 63.53; H, 7.14; N, 3.67.

Recrystallization from hexane $/ \mathrm{CH}_{2} \mathrm{Cl}_{2}$ afforded a single crystal that was suitable for X-ray analysis.

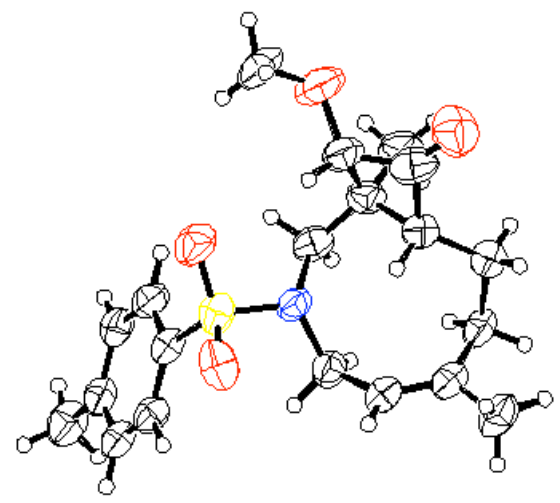

Figure S3. Molecular structure of rac-8 (CCDC-817443) in the solid state. Thermal ellipsoids are drawn at the $50 \%$ probability level. 


\section{6) Transannular reaction of epoxide $2 a$}

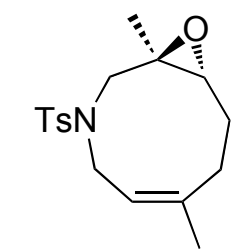

$(3 R, 4 R)-2 \mathbf{a}$

(>98\% dr, >98\% ee)

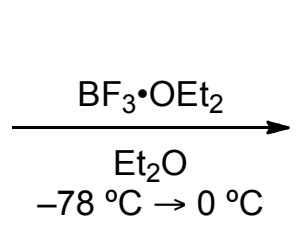

TsN

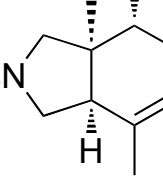

9 a

(>98\% dr, >98\% ee)

$73 \%$

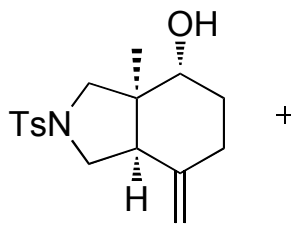

$9 \mathrm{~b}$

(>98\% dr, >98\% ee)

$17 \%$

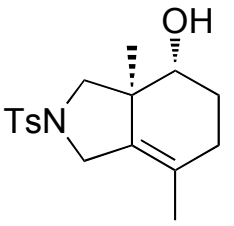

9c

(>98\% dr, >98\% ee)

$4 \%$

To a solution of $(3 R, 4 R)-\mathbf{2 a}(>98 \% \mathrm{dr},>98 \%$ ee; $53.8 \mathrm{mg}, 0.167 \mathrm{mmol})$ in dry $\mathrm{Et}_{2} \mathrm{O}(10 \mathrm{~mL})$ at $-78{ }^{\circ} \mathrm{C}$ was added $\mathrm{BF}_{3} \cdot \mathrm{OEt}_{2}(63.3 \mu \mathrm{L}, 0.502 \mathrm{mmol})$. The reaction mixture was allowed to warm up to $0{ }^{\circ} \mathrm{C}$ over a period of $1 \mathrm{~h}$ and was stirred for $2 \mathrm{~h}$. The reaction was quenched with saturated aqueous $\mathrm{NaHCO}_{3}$. The phases were separated and the aqueous layer was extracted with $\mathrm{Et}_{2} \mathrm{O}$. The combined organic phases were washed with brine, dried over $\mathrm{Na}_{2} \mathrm{SO}_{4}$, filtered and the solvent was removed in vacuo. The residue was purified by silica gel chromatography (hexane/AcOEt $=10 / 1$ to $2 / 1)$ to afford $48.3 \mathrm{mg}$ of a mixture of $\mathbf{9 a}$ and $\mathbf{9 b}\left(90 \% ; \mathbf{9 a} / \mathbf{9 b}=81 / 19\right.$ determined by ${ }^{1} \mathrm{H}$ NMR analysis) as a white solid and $2.1 \mathrm{mg}(4 \%)$ of $9 \mathbf{c}$ as a white solid. The mixture of $9 \mathbf{a}$ and $9 \mathbf{b}$ were separated by silica gel HPLC; column: Kanto Mightysil Si $60(5 \mu \mathrm{m})(2.0 \mathrm{~cm} \times 25 \mathrm{~cm})$, eluent: hexane/AcOEt $=$ 2/1, flow rate: $7.0 \mathrm{~mL} / \mathrm{min}$, detection: UV $254 \mathrm{~nm}$, temperature: rt. All of transannular products $9 \mathbf{a}, \mathbf{9 b}$ and $9 \mathbf{c}$ were determined as a single stereoisomer ( $>98 \% \mathrm{dr}$ analyzed by ${ }^{1} \mathrm{H}$ NMR, $>98 \%$ ee analyzed by chiral HPLC).

\section{3a,7-dimethyl-2-(p-toluenesulfonyl)-2,3,3a,4,5,7a-hexahydro-1H-isoindol-4-ol (9a)}

${ }^{1} \mathrm{H}$ NMR $\left(300 \mathrm{MHz}, \mathrm{CDCl}_{3}\right): \delta 7.72(\mathrm{~d}, J=8.1 \mathrm{~Hz}, 2 \mathrm{H}), 7.33(\mathrm{~d}, J=8.1 \mathrm{~Hz}, 2 \mathrm{H}), 5.24(\mathrm{~d}, J=5.1 \mathrm{~Hz}$, $1 \mathrm{H}), 3.71(\mathrm{~d}, J=10.2 \mathrm{~Hz}, 1 \mathrm{H}), 3.63(\mathrm{dd}, J=9.6,9.3 \mathrm{~Hz}, 1 \mathrm{H}), 3.41$ (dd, $J=9.9,6.0 \mathrm{~Hz}, 1 \mathrm{H}), 2.91$ (dd, $J=9.6,9.4 \mathrm{~Hz}, 1 \mathrm{H}), 2.86(\mathrm{~d}, J=10.2 \mathrm{~Hz}, 1 \mathrm{H}), 2.44(\mathrm{~s}, 3 \mathrm{H}), 2.24(\mathrm{dd}, J=9.4,9.3 \mathrm{~Hz}, 1 \mathrm{H})$, 2.21-2.12 (m, 1H), 2.03-1.94 (m, 1H), $1.92(\mathrm{~s}, 1 \mathrm{H}), 1.60(\mathrm{~s}, 3 \mathrm{H}), 0.91(\mathrm{~s}, 3 \mathrm{H}) ;{ }^{13} \mathrm{C} \mathrm{NMR}(75 \mathrm{MHz}$, $\left.\mathrm{CDCl}_{3}\right): \delta 143.4,133.7,132.1,129.6,127.4,119.7,67.2,56.7,52.7,51.0,44.7,31.3,22.8,21.7$, 16.2; IR (neat, $\mathrm{cm}^{-1}$ ): 3518, 2965, 2921, 2879, 1598, 1441, 1339, 1159, 1093, 1056, 813, 686, 663; Mp: $104{ }^{\circ} \mathrm{C}$; Analytical HPLC [column: CHIRALCEL OD-H $(4.6 \mathrm{~mm} \times 25 \mathrm{~cm})$, eluent: hexane $/ i-\mathrm{PrOH}=15 / 1$, flow rate: $0.5 \mathrm{~mL} / \mathrm{min}$, detection: $\mathrm{UV} 254 \mathrm{~nm}$, temperature: $\mathrm{rt}] t_{R}=27.2 \mathrm{~min}$ for (-)-isomer, $32.3 \mathrm{~min}$ for (+)-isomer; Optical rotation value: $[\alpha]_{\mathrm{D}}{ }^{27}-20.1\left(c 0.67, \mathrm{CHCl}_{3}\right)$ for $(-)$-isomer ( $>98 \%$ ee); HRMS (EI, positive): $m / z$ calcd for $\mathrm{C}_{17} \mathrm{H}_{23} \mathrm{NO}_{3} \mathrm{~S}[\mathrm{M}]^{+}: 321.1399$, found: 321.1389; Anal. calcd for $\mathrm{C}_{17} \mathrm{H}_{23} \mathrm{NO}_{3} \mathrm{~S} \cdot 1 / 5\left(\mathrm{C}_{6} \mathrm{H}_{14}\right)$ : C, 64.55; H, 7.68; N, 4.14. Found: $\mathrm{C}, 65.54 ; \mathrm{H}$, $7.65 ; \mathrm{N}, 4.09$.

\section{3a-methyl-7-methylene-2-(p-toluenesulfonyl)-octahydroisoindol-4-ol (9b)}

${ }^{1} \mathrm{H}$ NMR $\left(300 \mathrm{MHz}, \mathrm{CDCl}_{3}\right): \delta 7.73(\mathrm{~d}, J=8.0 \mathrm{~Hz}, 2 \mathrm{H}), 7.34(\mathrm{~d}, J=8.0 \mathrm{~Hz}, 2 \mathrm{H}), 4.81(\mathrm{~s}, 1 \mathrm{H}), 4.73$ (s, 1H), $3.76(\mathrm{~d}, J=9.8 \mathrm{~Hz}, 1 \mathrm{H}), 3.48(\mathrm{dd}, J=12.0,4.2 \mathrm{~Hz}, 1 \mathrm{H}), 3.34(\mathrm{dd}, J=10.2,9.9 \mathrm{~Hz}, 1 \mathrm{H})$, $3.26(\mathrm{dd}, J=10.7,10.2 \mathrm{~Hz}, 1 \mathrm{H}), 2.77$ (d, $J=9.8 \mathrm{~Hz}, 1 \mathrm{H}), 2.47$ (dd, $J=10.7,9.9 \mathrm{~Hz}, 1 \mathrm{H}), 2.45$ (s, $3 \mathrm{H}), 2.22-2.17(\mathrm{~m}, 2 \mathrm{H}), 1.76-1.69(\mathrm{~m}, 1 \mathrm{H}), 1.67(\mathrm{~s}, 1 \mathrm{H}), 1.54-1.39(\mathrm{~m}, 1 \mathrm{H}), 0.90(\mathrm{~s}, 3 \mathrm{H}) ;{ }^{13} \mathrm{C} \mathrm{NMR}$ $\left(75 \mathrm{MHz}, \mathrm{CDCl}_{3}\right): \delta 143.4,141.9,134.3,129.7,127.3,113.9,69.2,57.6,53.7,50.1,47.2,31.4,29.5$, 21.8, 15.2; IR (neat, $\mathrm{cm}^{-1}$ ): 3517, 2936, 1651, 1598, 1444, 1338, 1156, 1094, 1066, 709, 663; Mp: $115{ }^{\circ} \mathrm{C}$; Analytical HPLC [column: CHIRALCEL OD-H $(4.6 \mathrm{~mm} \times 25 \mathrm{~cm})$, eluent: hexane $/ i$-PrOH $=15 / 1$, flow rate: $0.5 \mathrm{~mL} / \mathrm{min}$, detection: UV $254 \mathrm{~nm}$, temperature: $\mathrm{rt}] t_{R}=35.8 \mathrm{~min}$ for $(+)$-isomer, $43.0 \mathrm{~min}$ for (-)-isomer; Optical rotation value: $[\alpha]_{\mathrm{D}}{ }^{24} 24.4\left(c \quad 0.52, \mathrm{CHCl}_{3}\right)$ for $(+)$-isomer $(>98 \%$ ee); HRMS (EI, positive): $m / z$ calcd for $\mathrm{C}_{17} \mathrm{H}_{23} \mathrm{NO}_{3} \mathrm{~S}$ [M] $]^{+}: 321.1399$, found: 321.1401; Anal. calcd 
for $\mathrm{C}_{17} \mathrm{H}_{23} \mathrm{NO}_{3} \mathrm{~S}$ : C, 63.52; H, 7.21; N, 4.36. Found: C, 63.38; H, 7.20; N, 4.27.

\section{3a,7-dimethyl-2-(p-toluenesulfonyl)-2,3,3a,4,5,6-hexahydro- $1 H$-isoindol-4-ol (9c)}

${ }^{1} \mathrm{H}$ NMR $\left(300 \mathrm{MHz}, \mathrm{CDCl}_{3}\right): \delta 7.72(\mathrm{~d}, J=8.4 \mathrm{~Hz}, 2 \mathrm{H}), 7.32(\mathrm{~d}, J=8.4 \mathrm{~Hz}, 2 \mathrm{H}), 3.99(\mathrm{~d}, J=13.2$ $\mathrm{Hz}, 1 \mathrm{H}), 3.73(\mathrm{~d}, J=13.2 \mathrm{~Hz}, 1 \mathrm{H}), 3.57(\mathrm{~d}, J=8.9 \mathrm{~Hz}, 1 \mathrm{H}), 3.47(\mathrm{dd}, J=10.8,5.1 \mathrm{~Hz}, 1 \mathrm{H}), 2.75(\mathrm{~d}$, $J=8.9 \mathrm{~Hz}, 1 \mathrm{H}), 2.44$ (s, 3H), 2.10-2.01 (m, 2H), 1.75-1.64 (m, 2H), 1.49 (s, 3H), $0.98(\mathrm{~s}, 3 \mathrm{H}) ;{ }^{13} \mathrm{C}$ NMR (75 MHz, $\mathrm{CDCl}_{3}$ ): $\delta 143.3,133.7,132.0,129.6,127.6,125.3,74.1,60.9,49.1,45.7,30.9,27.7$, 21.8, 19.1, 18.0; IR (neat, $\mathrm{cm}^{-1}$ ): 3513, 2936, 1725, 1598, 1465, 1342, 1159, 1094, 1047, 814, 709, 667; Analytical HPLC [column: CHIRALCEL OD-H $(4.6 \mathrm{~mm} \times 25 \mathrm{~cm})$, eluent: hexane $/ i$-PrOH $=$ 15/1, flow rate: $0.5 \mathrm{~mL} / \mathrm{min}$, detection: UV $254 \mathrm{~nm}$, temperature: $\mathrm{rt}] t_{R}=50.4 \mathrm{~min}, 54.5 \mathrm{~min}$; HRMS (EI, positive): $m / z$ calcd for $\mathrm{C}_{17} \mathrm{H}_{23} \mathrm{NO}_{3} \mathrm{~S}[\mathrm{M}]^{+}: 321.1399$, found: 321.1403 .

\section{7) Transannular reaction of cyclobutanation product 8}
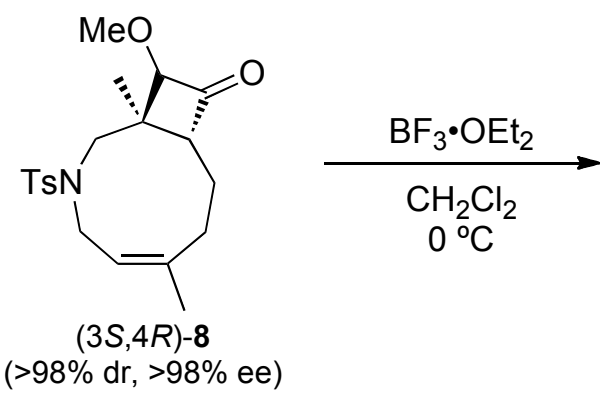

$(>98 \% \mathrm{dr},>98 \%$ ee $)$

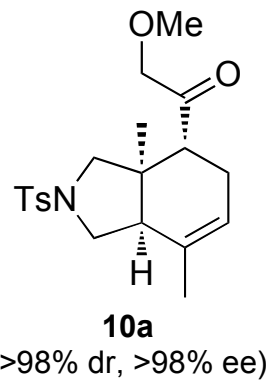

$52 \%$

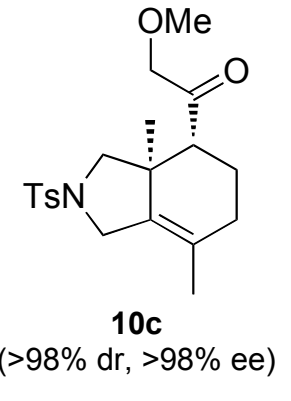

$18 \%$

To a solution of $(3 S, 4 R)-8(>98 \% \mathrm{dr},>98 \%$ ee; $8.5 \mathrm{mg}, 0.0225 \mathrm{mmol})$ in dry $\mathrm{CH}_{2} \mathrm{Cl}_{2}(5 \mathrm{~mL})$ at $0{ }^{\circ} \mathrm{C}$ was added $\mathrm{BF}_{3} \cdot \mathrm{OEt}_{2}(28.4 \mu \mathrm{L}, 0.225 \mathrm{mmol})$. The reaction mixture was stirred at that temperature for $24 \mathrm{~h}$. The reaction was quenched with saturated aqueous $\mathrm{NaHCO}_{3}$. The phases were separated and the aqueous layer was extracted with $\mathrm{CH}_{2} \mathrm{Cl}_{2}$. The combined organic phases were dried over $\mathrm{Na}_{2} \mathrm{SO}_{4}$, filtered and the solvent was removed in vacuo. The residue was purified by silica gel chromatography (hexane/AcOEt $=10 / 1$ to $2 / 1)$ to afford $4.4 \mathrm{mg}(52 \%)$ of $\mathbf{1 0 a}$ as a white solid and $1.5 \mathrm{mg}(18 \%)$ of $10 \mathrm{c}$ as a colorless oil. Both transannular products 10a and 10c were determined as a single stereoisomer ( $>98 \% \mathrm{dr}$ analyzed by ${ }^{1} \mathrm{H} \mathrm{NMR},>98 \%$ ee analyzed by chiral HPLC).

\section{1-[3a,7-dimethyl-2-(p-toluenesulfonyl)-2,3,3a,4,5,7a-hexahydro-1 $H$-isoindol-4-yl]-2-methoxyeth anone (10a)}

${ }^{1} \mathrm{H}$ NMR $\left(300 \mathrm{MHz}, \mathrm{CDCl}_{3}\right): \delta 7.70(\mathrm{~d}, J=8.3 \mathrm{~Hz}, 2 \mathrm{H}), 7.34(\mathrm{~d}, J=8.3 \mathrm{~Hz}, 2 \mathrm{H}), 5.40-5.35(\mathrm{~m}, 1 \mathrm{H})$, $3.98(\mathrm{~s}, 2 \mathrm{H}), 3.59(\mathrm{dd}, J=9.4,9.2 \mathrm{~Hz}, 1 \mathrm{H}), 3.43(\mathrm{~d}, J=10.5 \mathrm{~Hz}, 1 \mathrm{H}), 3.39$ (s, $3 \mathrm{H}), 3.09$ (dd, $J=9.5$, $9.4 \mathrm{~Hz}, 1 \mathrm{H}), 2.85(\mathrm{~d}, J=10.5 \mathrm{~Hz}, 1 \mathrm{H}), 2.65(\mathrm{dd}, J=9.9,6.6 \mathrm{~Hz}, 1 \mathrm{H}), 2.45(\mathrm{~s}, 3 \mathrm{H}), 2.18-2.13$ (m, 2H), $2.03(\mathrm{dd}, J=9.5,9.2 \mathrm{~Hz}, 1 \mathrm{H}), 1.62(\mathrm{~s}, 3 \mathrm{H}), 1.04(\mathrm{~s}, 3 \mathrm{H}) ;{ }^{13} \mathrm{C} \mathrm{NMR}\left(75 \mathrm{MHz}, \mathrm{CDCl}_{3}\right): \delta 209.1$, $143.5,133.8,132.2,129.7,127.5,120.0,78.2,59.3,58.0,51.9,51.1,43.5,41.7,25.8,22.9,21.7$, 18.7; IR (neat, $\mathrm{cm}^{-1}$ ): 2926, 1726, 1598, 1449, 1344, 1160, 1093, 1055, 814, 665; Mp: $82{ }^{\circ} \mathrm{C}$; Analytical HPLC [column: CHIRALCEL OD-H $(4.6 \mathrm{~mm} \times 25 \mathrm{~cm})$, eluent: hexane $i$-PrOH $=10 / 1$, flow rate: $0.5 \mathrm{~mL} / \mathrm{min}$, detection: UV $254 \mathrm{~nm}$, temperature: $\mathrm{rt}] t_{R}=33.9 \mathrm{~min}$ for (-)-isomer, 36.1 min for (+)-isomer; Optical rotation value: $[\alpha]_{\mathrm{D}}{ }^{26} 5.31\left(c 0.31, \mathrm{CHCl}_{3}\right)$ for $(+)$-isomer $(>98 \%$ ee); HRMS (FAB, matrix: 3-nitrobenzyl alcohol, positive): $m / z$ calcd for $\mathrm{C}_{20} \mathrm{H}_{28} \mathrm{NO}_{4} \mathrm{~S}[\mathrm{M}+\mathrm{H}]^{+}: 378.1739$, found: 378.1733; Anal. calcd for $\mathrm{C}_{20} \mathrm{H}_{27} \mathrm{NO}_{4} \mathrm{~S}$ : C, 63.63; H, 7.21; N, 3.71; S, 8.49. Found: C, 63.38; H, 7.10; N, 3.64; S, 8.34.

Recrystallization from hexane/ $\mathrm{CH}_{2} \mathrm{Cl}_{2}$ afforded a single crystal that was suitable for X-ray analysis. 


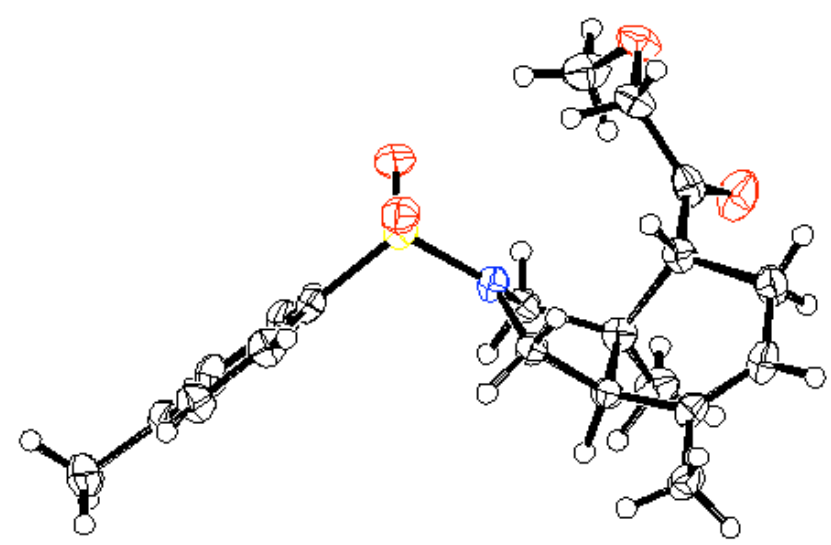

Figure S4. Molecular structure of (-)-10a (CCDC-817444) in the solid state. Thermal ellipsoids are drawn at the $50 \%$ probability level.

\section{1-[3a,7-dimethyl-2-(p-toluenesulfonyl)-2,3,3a,4,5,6-hexahydro-1 $H$-isoindol-4-yl]-2-methoxyetha none (10c)}

${ }^{1} \mathrm{H}$ NMR (300 MHz, $\left.\mathrm{CDCl}_{3}\right): \delta 7.73(\mathrm{~d}, J=8.1 \mathrm{~Hz}, 2 \mathrm{H}), 7.33(\mathrm{~d}, J=8.1 \mathrm{~Hz}, 2 \mathrm{H}), 4.02(\mathrm{~d}, J=17.1$ $\mathrm{Hz}, 1 \mathrm{H}), 3.96(\mathrm{~d}, J=17.1 \mathrm{~Hz}, 1 \mathrm{H}), 3.93(\mathrm{~d}, J=13.2 \mathrm{~Hz}, 1 \mathrm{H}), 3.78(\mathrm{~d}, J=13.2 \mathrm{~Hz}, 1 \mathrm{H}), 3.55(\mathrm{~d}, J=$ $9.0 \mathrm{~Hz}, 1 \mathrm{H}), 3.41(\mathrm{~s}, 3 \mathrm{H}), 2.90(\mathrm{~d}, J=9.0 \mathrm{~Hz}, 1 \mathrm{H}), 2.47-2.41(\mathrm{~m}, 1 \mathrm{H}), 2.44(\mathrm{~s}, 3 \mathrm{H}), 2.09-1.71(\mathrm{~m}$, 4H), $1.51(\mathrm{~s}, 3 \mathrm{H}), 0.87(\mathrm{~s}, 3 \mathrm{H}) ;{ }^{13} \mathrm{C} \mathrm{NMR}\left(75 \mathrm{MHz}, \mathrm{CDCl}_{3}\right): \delta 208.8,143.4,133.8,132.8,129.7$, 127.5, 125.5, 77.7, 60.8, 59.3, 50.4, 48.2, 42.7, 30.1, 21.7, 20.5, 20.2, 19.3; IR (neat, $\mathrm{cm}^{-1}$ ): 2921, 1720, 1598, 1450, 1343, 1159, 1094, 1058, 814, 709, 663; Analytical HPLC [column: CHIRALPAK AS-H $(4.6 \mathrm{~mm} \times 25 \mathrm{~cm})$, eluent: hexane/ $i$-PrOH $=2 / 1$, flow rate: $0.5 \mathrm{~mL} / \mathrm{min}$, detection: UV 254 $\mathrm{nm}$, temperature: $\mathrm{rt}] t_{R}=36.2 \mathrm{~min}$ for $(+)$-isomer, $56.4 \mathrm{~min}$ for (-)-isomer; Optical rotation value: $\left.[\alpha]_{\mathrm{D}}{ }^{23}-5.69(c) 0.22, \mathrm{CHCl}_{3}\right)$ for $(-)$-isomer ( $>98 \%$ ee); HRMS (EI, positive): $\mathrm{m} / z$ calcd for $\mathrm{C}_{20} \mathrm{H}_{27} \mathrm{NO}_{4} \mathrm{~S}[\mathrm{M}]^{+}:$377.1661, found: 377.1664; Anal. calcd for $\mathrm{C}_{20} \mathrm{H}_{27} \mathrm{NO}_{4} \mathrm{~S}: \mathrm{C}, 63.63 ; \mathrm{H}, 7.21$; N, 3.71. Found: C, 63.94; H, 7.07; N, 3.45.

\section{8) Transannular reaction of epoxide 3a}
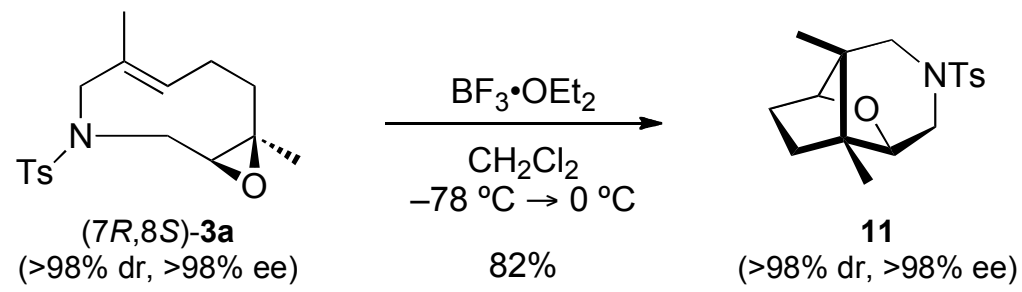

To a solution of $(7 R, 8 S)-3 \mathbf{a}(>98 \% \mathrm{dr},>98 \%$ ee; $19.9 \mathrm{mg}, 0.0619 \mathrm{mmol})$ in dry $\mathrm{CH}_{2} \mathrm{Cl}_{2}(10 \mathrm{~mL})$ at $-78{ }^{\circ} \mathrm{C}$ was added $\mathrm{BF}_{3} \cdot \mathrm{OEt}_{2}(11.7 \mu \mathrm{L}, 0.0929 \mathrm{mmol})$. The reaction mixture was allowed to warm up to room temperature over a period of $1 \mathrm{~h}$ and was stirred for $1 \mathrm{~h}$. The reaction was quenched with saturated aqueous $\mathrm{NaHCO}_{3}$. The phases were separated and the aqueous layer was extracted with $\mathrm{CH}_{2} \mathrm{Cl}_{2}$. The combined organic phases were dried over $\mathrm{Na}_{2} \mathrm{SO}_{4}$, filtered and the solvent was removed in vacuo. The residue was purified by silica gel chromatography (hexane/AcOEt $=20 / 1$ to $5 / 1)$ to afford $16.3 \mathrm{mg}$ of $\mathbf{1 1}\left(82 \%\right.$; $>98 \% \mathrm{dr}$ analyzed by ${ }^{1} \mathrm{H} \mathrm{NMR},>98 \%$ ee analyzed by chiral HPLC) as a white solid. 
${ }^{1} \mathrm{H}$ NMR $\left(300 \mathrm{MHz}, \mathrm{CDCl}_{3}\right): \delta 7.70(\mathrm{~d}, J=8.1 \mathrm{~Hz}, 2 \mathrm{H}), 7.30(\mathrm{~d}, J=8.1 \mathrm{~Hz}, 2 \mathrm{H}), 3.73(\mathrm{~d}, J=3.3 \mathrm{~Hz}$, $1 \mathrm{H}), 3.66$ (brs, $1 \mathrm{H}), 3.45$ (d, $J=12.6 \mathrm{~Hz}, 1 \mathrm{H}), 3.40$ (dd, $J=12.3,3.6 \mathrm{~Hz}, 1 \mathrm{H}), 3.08$ (d, $J=12.3,1.2$ $\mathrm{Hz}, 1 \mathrm{H}), 3.02(\mathrm{~d}, J=12.6 \mathrm{~Hz}, 1 \mathrm{H}), 2.43(\mathrm{~s}, 3 \mathrm{H}), 1.73-1.43(\mathrm{~m}, 4 \mathrm{H}), 0.92(\mathrm{~s}, 3 \mathrm{H}), 0.83(\mathrm{~s}, 3 \mathrm{H}) ;{ }^{13} \mathrm{C}$ $\operatorname{NMR}\left(75 \mathrm{MHz}, \mathrm{CDCl}_{3}\right): \delta 143.1,134.7,129.4,127.4,83.9,81.0,48.1,47.1,46.1,43.7,33.5,30.1$, 21.7, 15.0, 11.8; IR (reflection, $\mathrm{cm}^{-1}$ ): 2938, 1921, 1598, 1470, 1394, 1332, 1154, 1058, 1010, 815, 717, 592, 547; Mp: $92{ }^{\circ} \mathrm{C}$; Analytical HPLC [column: CHIRALCEL OD-H $(4.6 \mathrm{~mm} \times 25 \mathrm{~cm})$, eluent: hexane $/ i$-PrOH $=4 / 1$, flow rate: $0.5 \mathrm{~mL} / \mathrm{min}$, detection: UV $254 \mathrm{~nm}$, temperature: $\mathrm{rt}] t_{R}=$ $17.4 \mathrm{~min}$ for $(+)$-isomer, $24.9 \mathrm{~min}$ for $(-)$-isomer; Optical rotation value: $[\alpha]_{\mathrm{D}}{ }^{28}-16.3(c 0.36$, $\mathrm{CHCl}_{3}$ ) for (-)-isomer ( $>98 \%$ ee); HRMS (FAB, matrix: 3-nitrobenzyl alcohol, positive): $\mathrm{m} / \mathrm{z}$ calcd for $\mathrm{C}_{17} \mathrm{H}_{24} \mathrm{NO}_{3} \mathrm{~S}[\mathrm{M}+\mathrm{H}]^{+}:$322.1477, found: 322.1472; Anal. calcd for $\mathrm{C}_{17} \mathrm{H}_{23} \mathrm{NO}_{3} \mathrm{~S}$ : C, 63.52; $\mathrm{H}$, 7.21; N, 4.36. Found: C, 63.37; H, 7.03; N, 4.28.

Recrystallization from hexane afforded a single crystal that was suitable for X-ray analysis.

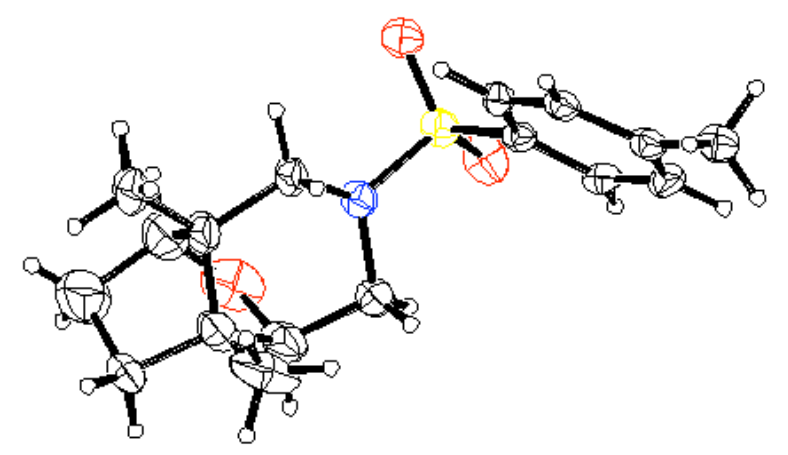

Figure S5. Molecular structure of rac-11 (CCDC-817445) in the solid state. Thermal ellipsoids are drawn at the $40 \%$ probability level.

\section{9) Transannular reaction of cyclic amide 1a using NBS}<smiles>CC1=CCN([13F])CC(C)=CCC1</smiles>

$(R)-\mathbf{1 a}$ $(>98 \%$ ee $)$

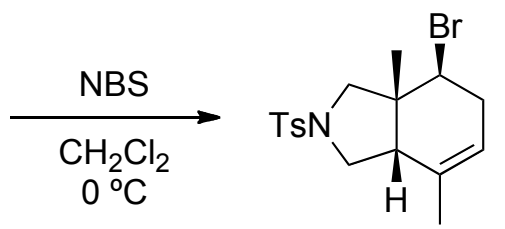

$12 a$ $(>98 \% \mathrm{dr},>98 \%$ ee) $53 \%$

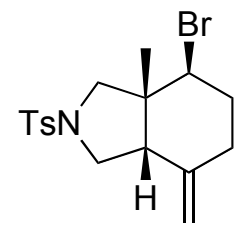

12b $(>98 \% \mathrm{dr},>98 \%$ ee) $18 \%$

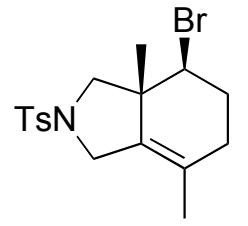

12c

$(>98 \%$ dr, $>98 \%$ ee)

$27 \%$

To a solution of $(R)-\mathbf{1 a}(>98 \%$ ee; $20.0 \mathrm{mg}, 0.0655 \mathrm{mmol})$ in dry $\mathrm{CH}_{2} \mathrm{Cl}_{2}(5 \mathrm{~mL})$ at $0{ }^{\circ} \mathrm{C}$ was added $N$-bromosuccinimide $(17.5 \mathrm{mg}, 0.0982 \mathrm{mmol})$. The mixture was stirred at that temperature for $3 \mathrm{~h}$. The reaction was quenched with saturated aqueous $\mathrm{NaHCO}_{3}$. The phases were separated and the aqueous layer was extracted with $\mathrm{CH}_{2} \mathrm{Cl}_{2}$. The combined organic phases were dried over $\mathrm{Na}_{2} \mathrm{SO}_{4}$, filtered and the solvent was removed in vacuo. The residue was purified by silica gel chromatography (hexane/AcOEt $=1 / 0$ to $12 / 1$ ) to afford $24.7 \mathrm{mg}$ of a mixture of $\mathbf{1 2 a}, \mathbf{1 2 b}$ and 12c $\left(98 \% ; \mathbf{1 2 a} / \mathbf{1 2 b} / \mathbf{1 2 c}=54 / 19 / 27\right.$ determined by ${ }^{1} \mathrm{H}$ NMR analysis $)$ as a white solid. The mixture of 12a, 12b and 12c were separated by silica gel HPLC; column: Kanto Mightysil Si $60(5 \mu \mathrm{m})(2.0 \mathrm{~cm}$ $\times 25 \mathrm{~cm}$ ), eluent: hexane $/$ AcOEt $=9 / 1$, flow rate: $7.0 \mathrm{~mL} / \mathrm{min}$, detection: UV $254 \mathrm{~nm}$, temperature: rt. All of transannular products 12a, 12b and 12c were determined as a single stereoisomer ( $>98 \% \mathrm{dr}$ analyzed by ${ }^{1} \mathrm{H}$ NMR, $>98 \%$ ee analyzed by chiral HPLC). 


\section{4-bromo-3a,7-dimethyl-2-(p-toluenesulfonyl)-2,3,3a,4,5,7a-hexahydro-1 $H$-isoindole (12a)}

${ }^{1} \mathrm{H}$ NMR (300 MHz, $\left.\mathrm{CDCl}_{3}\right): \delta 7.73(\mathrm{~d}, J=8.1 \mathrm{~Hz}, 2 \mathrm{H}), 7.34(\mathrm{~d}, J=8.1 \mathrm{~Hz}, 2 \mathrm{H}), 5.21$ (brs, 1H), $3.81(\mathrm{dd}, J=8.7,7.8 \mathrm{~Hz}, 1 \mathrm{H}), 3.76(\mathrm{~d}, J=10.5 \mathrm{~Hz}, 1 \mathrm{H}), 3.68(\mathrm{dd}, J=9.5,9.2 \mathrm{~Hz}, 1 \mathrm{H}), 3.00(\mathrm{~d}, J=$ $10.5 \mathrm{~Hz}, 1 \mathrm{H}), 2.87(\mathrm{dd}, J=10.1,9.5 \mathrm{~Hz}, 1 \mathrm{H}), 2.49-2.45(\mathrm{~m}, 2 \mathrm{H}), 2.45(\mathrm{~s}, 3 \mathrm{H}), 2.41$ (dd, $J=10.1,9.2$ $\mathrm{Hz}, 1 \mathrm{H}), 1.60(\mathrm{~d}, J=1.5 \mathrm{~Hz}, 3 \mathrm{H}), 1.07(\mathrm{~s}, 3 \mathrm{H}) ;{ }^{13} \mathrm{C} \mathrm{NMR}\left(75 \mathrm{MHz}, \mathrm{CDCl}_{3}\right): \delta 143.6,133.5,132.3$, 129.7, 127.5, 120.8, 59.1, 54.8, 52.4, 50.7, 45.4, 34.3, 22.7, 21.8, 18.8; IR (neat, $\mathrm{cm}^{-1}$ ): 2970, 1440, 1345, 1160, 1093, 1051, 814, 691, 664, 604, 582, 548, 528; Mp: $142{ }^{\circ} \mathrm{C}$; Analytical HPLC [column: CHIRALCEL OD-H $(4.6 \mathrm{~mm} \times 25 \mathrm{~cm})$, eluent: hexane $/ i-\mathrm{PrOH}=10 / 1$, flow rate: $0.5 \mathrm{~mL} / \mathrm{min}$, detection: UV $254 \mathrm{~nm}$, temperature: $\mathrm{rt}] t_{R}=22.4 \mathrm{~min}$ for (+)-isomer, $23.1 \mathrm{~min}$ for (-)-isomer; Optical rotation value: $[\alpha]_{\mathrm{D}}^{23} 57.8\left(c 0.50, \mathrm{CHCl}_{3}\right)$ for $(+)$-isomer $(>98 \%$ ee); HRMS (FAB, matrix: 3-nitrobenzyl alcohol, positive): $m / z$ calcd for $\mathrm{C}_{17} \mathrm{H}_{23} \mathrm{BrNO}_{2} \mathrm{~S}[\mathrm{M}+\mathrm{H}]^{+}: 384.0633$, found: 384.0631; Anal. calcd for $\mathrm{C}_{17} \mathrm{H}_{22} \mathrm{BrNO}_{2} \mathrm{~S}$ : C, 53.13; H, 5.77; N, 3.64. Found: $\mathrm{C}, 53.04 ; \mathrm{H}, 5.78 ; \mathrm{N}, 3.62$.

Recrystallization from hexane $/ \mathrm{CH}_{2} \mathrm{Cl}_{2}$ afforded a single crystal that was suitable for X-ray analysis.

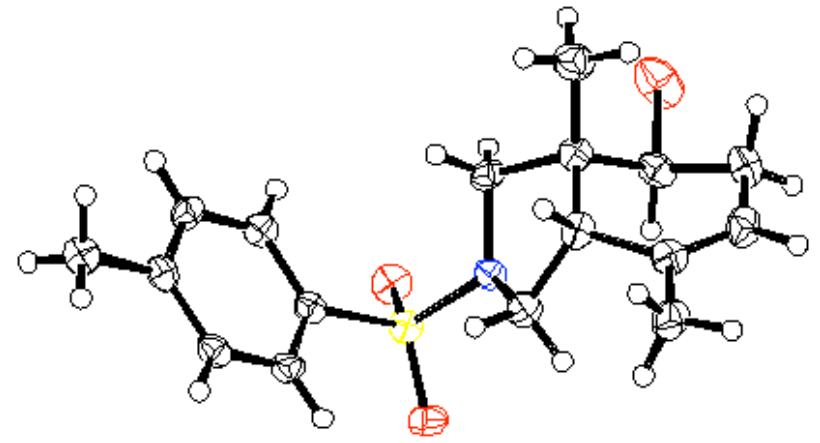

Figure S6. Molecular structure of rac-12a (CCDC-817446) in the solid state. Thermal ellipsoids are drawn at the $50 \%$ probability level.

\section{4-bromo-3a-methyl-7-methylene-2-(p-toluenesulfonyl)octahydroisoindole (12b)}

${ }^{1} \mathrm{H}$ NMR $\left(300 \mathrm{MHz}, \mathrm{CDCl}_{3}\right.$ ): $\delta 7.75(\mathrm{~d}, J=8.3 \mathrm{~Hz}, 2 \mathrm{H}), 7.35(\mathrm{~d}, J=8.3 \mathrm{~Hz}, 2 \mathrm{H}), 4.84(\mathrm{~s}, 1 \mathrm{H}), 4.78$ (s, 1H), $3.88(\mathrm{dd}, J=12.9,4.2 \mathrm{~Hz}, 1 \mathrm{H}), 3.78(\mathrm{~d}, J=10.2 \mathrm{~Hz}, 1 \mathrm{H}), 3.35$ (dd, $J=10.4,9.8 \mathrm{~Hz}, 1 \mathrm{H})$, $3.25(\mathrm{dd}, J=10.8,10.4 \mathrm{~Hz}, 1 \mathrm{H}), 2.86(\mathrm{~d}, J=10.2 \mathrm{~Hz}, 1 \mathrm{H}), 2.67$ (dd, $J=10.8,9.8 \mathrm{~Hz}, 1 \mathrm{H}), 2.46$ (s, $3 \mathrm{H}), 2.20-2.05(\mathrm{~m}, 3 \mathrm{H}), 1.98-1.83(\mathrm{~m}, 1 \mathrm{H}), 1.08(\mathrm{~s}, 3 \mathrm{H}) ;{ }^{13} \mathrm{C} \mathrm{NMR}\left(75 \mathrm{MHz}, \mathrm{CDCl}_{3}\right): \delta 143.6,140.4$, 134.0, 129.8, 127.4, 114.8, 59.2, 56.1, 54.1, 49.5, 48.0, 34.1, 31.7, 21.8, 18.5; IR (neat, $\mathrm{cm}^{-1}$ ): 2945 , 1597, 1473, 1345, 1304, 1168, 1092, 1044, 892, 810, 708, 664, 598; Mp: $170{ }^{\circ} \mathrm{C}$; Analytical HPLC [column: CHIRALCEL OD-H $(4.6 \mathrm{~mm} \times 25 \mathrm{~cm})$, eluent: hexane $/ i-\mathrm{PrOH}=10 / 1$, flow rate: 0.5 $\mathrm{mL} / \mathrm{min}$, detection: UV $254 \mathrm{~nm}$, temperature: $\mathrm{rt}] t_{R}=25.1 \mathrm{~min}$ for (-)-isomer, $29.1 \mathrm{~min}$ for $(+)$-isomer; Optical rotation value: $[\alpha]_{\mathrm{D}}{ }^{22}-20.2\left(c 0.16, \mathrm{CHCl}_{3}\right)$ for $(-)$-isomer $(>98 \%$ ee); HRMS (EI, positive): $m / z$ calcd for $\mathrm{C}_{17} \mathrm{H}_{22} \mathrm{BrNO}_{2} \mathrm{~S}[\mathrm{M}]^{+}:$383.0555, found: 383.0557 .

\section{4-bromo-3a,7-dimethyl-2-(p-toluenesulfonyl)-2,3,3a,4,5,6-hexahydro- $1 \mathrm{H}$-isoindole (12c)}

${ }^{1} \mathrm{H}$ NMR $\left(300 \mathrm{MHz}, \mathrm{CDCl}_{3}\right): \delta 7.72(\mathrm{~d}, J=8.1 \mathrm{~Hz}, 2 \mathrm{H}), 7.34(\mathrm{~d}, J=8.1 \mathrm{~Hz}, 2 \mathrm{H}), 4.06(\mathrm{~d}, J=13.5$ Hz, 1H), 3.90 (dd, $J=10.2,5.7 \mathrm{~Hz}, 1 \mathrm{H}), 3.77(\mathrm{~d}, J=13.5 \mathrm{~Hz}, 1 \mathrm{H}), 3.53$ (d, $J=9.0 \mathrm{~Hz}, 1 \mathrm{H}), 2.69$ (d, $J=9.0 \mathrm{~Hz}, 1 \mathrm{H}), 2.45(\mathrm{~s}, 3 \mathrm{H}), 2.23-2.02(\mathrm{~m}, 4 \mathrm{H}), 1.45(\mathrm{~s}, 3 \mathrm{H}), 1.15(\mathrm{~s}, 3 \mathrm{H}) ;{ }^{13} \mathrm{C} \mathrm{NMR}(75 \mathrm{MHz}$, $\left.\mathrm{CDCl}_{3}\right): \delta 143.5,133.5,131.8,129.7,127.6,125.4,61.4,57.3,49.8,46.0,32.0,30.0,21.8,20.5$, 19.1; IR (neat, $\mathrm{cm}^{-1}$ ): 2917, 1598, 1449, 1346, 1160, 1095, 1053, 1000, 813, 709, 681, 660; Mp: $154{ }^{\circ} \mathrm{C}$; Analytical HPLC [column: CHIRALCEL OD-H $(4.6 \mathrm{~mm} \times 25 \mathrm{~cm})$, eluent: hexane $/ i$-PrOH $=10 / 1$, flow rate: $0.5 \mathrm{~mL} / \mathrm{min}$, detection: UV $254 \mathrm{~nm}$, temperature: $\mathrm{rt}] t_{R}=19.3 \mathrm{~min}$ for (-)-isomer, $20.2 \mathrm{~min}$ for (+)-isomer; Optical rotation value: $[\alpha]_{\mathrm{D}}{ }^{23}-1.19\left(c 0.30, \mathrm{CHCl}_{3}\right)$ for $(-)$-isomer $(>98 \%$ ee); HRMS (EI, positive): $m / z$ calcd for $\mathrm{C}_{17} \mathrm{H}_{22} \mathrm{BrNO}_{2} \mathrm{~S}[\mathrm{M}]^{+}$: 383.0555, found: 383.0551; Anal. 
calcd for $\mathrm{C}_{17} \mathrm{H}_{22} \mathrm{BrNO}_{2} \mathrm{~S}: \mathrm{C}, 53.13 ; \mathrm{H}, 5.77 ; \mathrm{N}, 3.64$. Found: C, 53.03; H, 5.78; N, 3.61.

Recrystallization from hexane/ $\mathrm{CH}_{2} \mathrm{Cl}_{2}$ afforded a single crystal that was suitable for X-ray analysis.

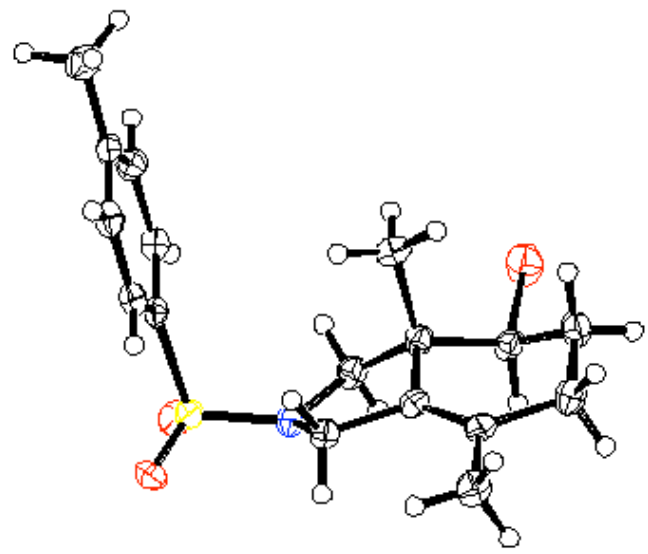

Figure S7. Molecular structure of (-)-12c (CCDC-817447) in the solid state. Thermal ellipsoids are drawn at the $50 \%$ probability level.

10) Transannular reaction of cyclic amide 1a using NIS<smiles>CC1=CCN([As])CC(C)=CCC1</smiles>

$(S)-1 \mathbf{a}$

$(>98 \%$ ee $)$

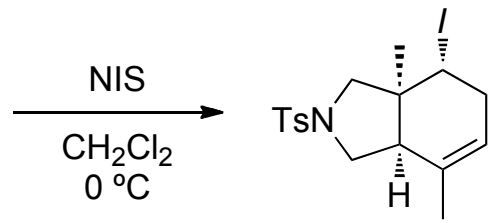

$13 a$ (>98\% dr, >98\% ee)

$53 \%$

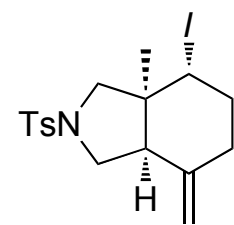

$13 b$ (>98\% dr, >98\% ee)

$31 \%$

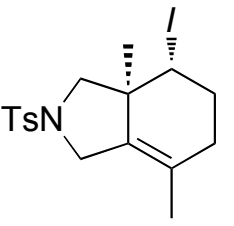

$13 \mathrm{c}$

(>98\% dr, >98\% ee)

$13 \%$

To a solution of $(S)-1 \mathrm{a}(>98 \%$ ee; $20.0 \mathrm{mg}, 0.0655 \mathrm{mmol})$ in dry $\mathrm{CH}_{2} \mathrm{Cl}_{2}(5 \mathrm{~mL})$ at $0{ }^{\circ} \mathrm{C}$ was added $N$-iodosuccinimide $(22.1 \mathrm{mg}, 0.0982 \mathrm{mmol})$. The mixture was stirred at that temperature for $2.5 \mathrm{~h}$. The reaction was quenched with saturated aqueous $\mathrm{NaHCO}_{3}$. The phases were separated and the aqueous layer was extracted with $\mathrm{CH}_{2} \mathrm{Cl}_{2}$. The combined organic phases were dried over $\mathrm{Na}_{2} \mathrm{SO}_{4}$, filtered and the solvent was removed in vacuo. The residue was purified by silica gel chromatography (hexane/AcOEt $=1 / 0$ to $12 / 1$ ) to afford $27.4 \mathrm{mg}$ of a mixture of $\mathbf{1 3 a}, \mathbf{1 3 b}$ and 13c $\left(97 \% ; \mathbf{1 3 a} / \mathbf{1 3 b} / \mathbf{1 3 c}=55 / 32 / 13\right.$ determined by ${ }^{1} \mathrm{H}$ NMR analysis) as a white solid. The mixture of 13a, 13b and 13c were separated by silica gel HPLC; column: Kanto Mightysil Si $60(5 \mu \mathrm{m})(2.0 \mathrm{~cm}$ $\times 25 \mathrm{~cm}$ ), eluent: hexane $/$ AcOEt $=9 / 1$, flow rate: $7.0 \mathrm{~mL} / \mathrm{min}$, detection: UV $254 \mathrm{~nm}$, temperature: rt. All of transannular products 13a, 13b and 13c were determined as a single stereoisomer ( $>98 \% \mathrm{dr}$ analyzed by ${ }^{1} \mathrm{H} \mathrm{NMR},>98 \%$ ee analyzed by chiral HPLC).

\section{4-iodo-3a,7-dimethyl-2-(p-toluenesulfonyl)-2,3,3a,4,5,7a-hexahydro-1H-isoindole (13a)}

${ }^{1} \mathrm{H}$ NMR $\left(300 \mathrm{MHz}, \mathrm{CDCl}_{3}\right): \delta 7.74(\mathrm{~d}, J=8.1 \mathrm{~Hz}, 2 \mathrm{H}), 7.35(\mathrm{~d}, J=8.1 \mathrm{~Hz}, 2 \mathrm{H}), 5.11$ (brs, $\left.1 \mathrm{H}\right)$, $3.98(\mathrm{dd}, J=11.1,6.0 \mathrm{~Hz}, 1 \mathrm{H}), 3.68(\mathrm{dd}, J=9.6,9.0 \mathrm{~Hz}, 1 \mathrm{H}), 3.61(\mathrm{~d}, J=10.8 \mathrm{~Hz}, 1 \mathrm{H}), 3.05(\mathrm{~d}, J=$ $10.8 \mathrm{~Hz}, 1 \mathrm{H}), 2.88(\mathrm{dd}, J=9.8,9.6 \mathrm{~Hz}, 1 \mathrm{H}), 2.76-2.52(\mathrm{~m}, 2 \mathrm{H}), 2.46$ (s, 3H), 2.43 (dd, $J=9.8,9.0$ $\mathrm{Hz}, 1 \mathrm{H}), 1.60(\mathrm{~d}, J=0.9 \mathrm{~Hz}, 3 \mathrm{H}), 1.09$ (s, 3H); ${ }^{13} \mathrm{C} \mathrm{NMR}\left(75 \mathrm{MHz}, \mathrm{CDCl}_{3}\right): \delta 143.6,133.6,132.3$, 129.8, 127.5, 121.9, 61.7, 52.5, 48.7, 45.3, 37.1, 36.9, 22.8, 21.8, 21.5; IR (neat, $\mathrm{cm}^{-1}$ ): 2967, 1438, 1345, 1159, 1092, 1051, 813, 709, 685, 662, 600, 574, 549; Mp: $91{ }^{\circ} \mathrm{C}$; Analytical HPLC [column: CHIRALCEL OD-H $(4.6 \mathrm{~mm} \times 25 \mathrm{~cm})$, eluent: hexane $/ i-\mathrm{PrOH}=10 / 1$, flow rate: $0.5 \mathrm{~mL} / \mathrm{min}$, detection: UV $254 \mathrm{~nm}$, temperature: $\mathrm{rt}] t_{R}=22.4 \mathrm{~min}$ for (+)-isomer, $23.1 \mathrm{~min}$ for (-)-isomer; Optical rotation value: $[\alpha]_{\mathrm{D}}{ }^{23}-54.0\left(c 1.16, \mathrm{CHCl}_{3}\right)$ for $(-)$-isomer $(>98 \%$ ee); HRMS (FAB, matrix: 3-nitrobenzyl alcohol, positive): $m / z$ calcd for $\mathrm{C}_{17} \mathrm{H}_{23} \mathrm{INO}_{2} \mathrm{~S}[\mathrm{M}+\mathrm{H}]^{+}$: 432.0494, found: 432.0496; Anal. calcd for $\mathrm{C}_{17} \mathrm{H}_{22} \mathrm{INO}_{2} \mathrm{~S}$ : C, 47.34; H, 5.14; N, 3.25. Found: C, 47.53; H, 5.14; N, 3.00 . 


\section{4-iodo-3a-methyl-7-methylene-2-(p-toluenesulfonyl)octahydroisoindole (13b)}

${ }^{1} \mathrm{H}$ NMR (300 MHz, $\mathrm{CDCl}_{3}$ ): $\delta 7.75(\mathrm{~d}, J=8.1 \mathrm{~Hz}, 2 \mathrm{H}), 7.36(\mathrm{~d}, J=8.1 \mathrm{~Hz}, 2 \mathrm{H}), 4.82(\mathrm{~s}, 1 \mathrm{H}), 4.77$ $(\mathrm{s}, 1 \mathrm{H}), 4.06(\mathrm{dd}, J=12.0,4.2 \mathrm{~Hz}, 1 \mathrm{H}), 3.64(\mathrm{~d}, J=10.2 \mathrm{~Hz}, 1 \mathrm{H}), 3.34(\mathrm{dd}, J=10.1,9.8 \mathrm{~Hz}, 1 \mathrm{H})$, $3.27(\mathrm{dd}, J=10.8,10.1 \mathrm{~Hz}, 1 \mathrm{H}), 2.88(\mathrm{~d}, J=10.2 \mathrm{~Hz}, 1 \mathrm{H}), 2.73(\mathrm{dd}, J=10.8,9.8 \mathrm{~Hz}, 1 \mathrm{H}), 2.47$ (s, $3 \mathrm{H}), 2.28-1.98(\mathrm{~m}, 4 \mathrm{H}), 1.12(\mathrm{~s}, 3 \mathrm{H}) ;{ }^{13} \mathrm{C} \mathrm{NMR}\left(75 \mathrm{MHz}, \mathrm{CDCl}_{3}\right): \delta 143.6,140.5,134.0,129.9$, 127.4, 114.7, 61.2, 52.6, 49.4, 48.1, 38.1, 36.8, 32.9, 21.82, 21.80; IR (neat, $\mathrm{cm}^{-1}$ ): 2942, 1598, 1441, 1345, 1167, 1092, 1045, 907, 809, 680, 662, 597; Mp: $155{ }^{\circ} \mathrm{C}$; Analytical HPLC [column: CHIRALCEL OD-H $(4.6 \mathrm{~mm} \times 25 \mathrm{~cm})$, eluent: hexane $/ i-\mathrm{PrOH}=10 / 1$, flow rate: $0.5 \mathrm{~mL} / \mathrm{min}$, detection: UV $254 \mathrm{~nm}$, temperature: $\mathrm{rt}] t_{R}=25.6 \mathrm{~min}$ for (-)-isomer, $27.8 \mathrm{~min}$ for (+)-isomer; Optical rotation value: $[\alpha]_{\mathrm{D}}^{22} 26.7\left(c 0.84, \mathrm{CHCl}_{3}\right)$ for $(+)$-isomer ( $>98 \%$ ee); HRMS (EI, positive): $m / z$ calcd for $\mathrm{C}_{17} \mathrm{H}_{22} \mathrm{INO}_{2} \mathrm{~S}[\mathrm{M}]^{+}: 431.0416$, found:431.0421; Anal. calcd for $\mathrm{C}_{17} \mathrm{H}_{22} \mathrm{INO}_{2} \mathrm{~S}$ : C, 47.34; H, 5.14; N, 3.25. Found: C, 47.49; H, 5.16; N, 3.18.

Recrystallization from hexane $/ \mathrm{CH}_{2} \mathrm{Cl}_{2}$ afforded a single crystal that was suitable for X-ray analysis.

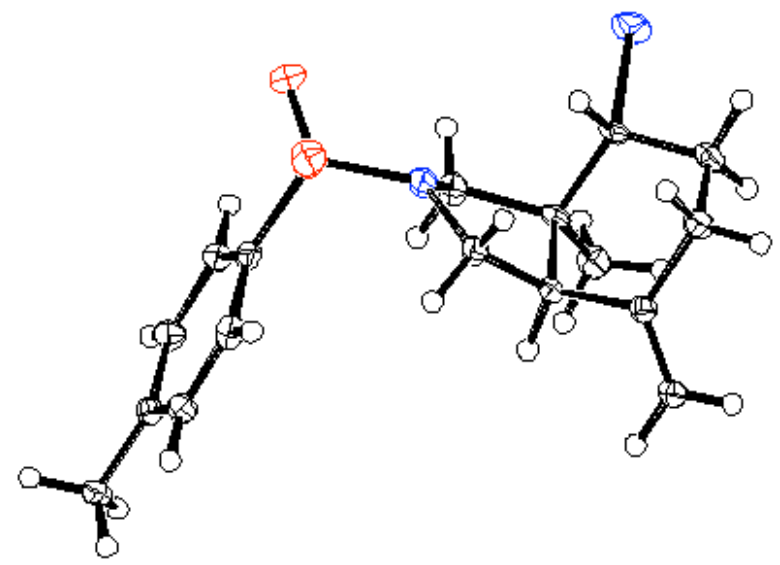

Figure S8. Molecular structure of rac-13b (CCDC-818009) in the solid state. Thermal ellipsoids are drawn at the $50 \%$ probability level.

\section{4-iodo-3a,7-dimethyl-2-(p-toluenesulfonyl)-2,3,3a,4,5,6-hexahydro-1H-isoindole (13c)}

${ }^{1} \mathrm{H}$ NMR $\left(300 \mathrm{MHz}, \mathrm{CDCl}_{3}\right): \delta 7.72(\mathrm{~d}, J=8.1 \mathrm{~Hz}, 2 \mathrm{H}), 7.34(\mathrm{~d}, J=8.1 \mathrm{~Hz}, 2 \mathrm{H}), 4.09(\mathrm{~d}, J=13.4$ $\mathrm{Hz}, 1 \mathrm{H}), 4.02(\mathrm{dd}, J=12.0,5.1 \mathrm{~Hz}, 1 \mathrm{H}), 3.81(\mathrm{~d}, J=13.4 \mathrm{~Hz}, 1 \mathrm{H}), 3.48$ (d, $J=9.0 \mathrm{~Hz}, 1 \mathrm{H}), 2.63$ (d, $J=9.0 \mathrm{~Hz}, 1 \mathrm{H}), 2.45(\mathrm{~s}, 3 \mathrm{H}), 2.38-2.24(\mathrm{~m}, 2 \mathrm{H}), 2.14-2.00(\mathrm{~m}, 1 \mathrm{H}), 1.94-1.83(\mathrm{~m}, 1 \mathrm{H}), 1.48(\mathrm{~s}, 3 \mathrm{H})$, $1.18(\mathrm{~s}, 3 \mathrm{H}) ;{ }^{13} \mathrm{C}$ NMR $\left(75 \mathrm{MHz}, \mathrm{CDCl}_{3}\right): \delta 143.5,133.5,130.3,129.7,127.6,125.4,62.6,50.2$, 45.7, 36.7, 32.6, 32.4, 22.9, 21.8, 19.2; IR (neat, $\mathrm{cm}^{-1}$ ): 2967, 1598, 1447, 1346, 1160, 1095, 1051, 812, 676, 658; Mp: $154{ }^{\circ} \mathrm{C}$; Analytical HPLC [column: CHIRALCEL OD-H $(4.6 \mathrm{~mm} \times 25 \mathrm{~cm})$, eluent: hexane $/ i$-PrOH $=10 / 1$, flow rate: $0.5 \mathrm{~mL} / \mathrm{min}$, detection: UV $254 \mathrm{~nm}$, temperature: $\mathrm{rt}] t_{R}=$ $19.4 \mathrm{~min}$ for (+)-isomer, $20.8 \mathrm{~min}$ for (-)-isomer; Optical rotation value: $[\alpha]_{\mathrm{D}}{ }^{24}-45.4(c 0.15$, $\mathrm{CHCl}_{3}$ ) for (-)-isomer ( $>98 \%$ ee); 3-nitrobenzyl alcohol, positive): $\mathrm{m} / z$ calcd for $\mathrm{C}_{17} \mathrm{H}_{23} \mathrm{INO}_{2} \mathrm{~S}$ $[\mathrm{M}+\mathrm{H}]^{+}:$432.0494, found: 432.0494 . 
11) Preparation of $N$-(trifluoromethanesulfonyl)phosphoramidic acid diphenyl ester ${ }^{1}$

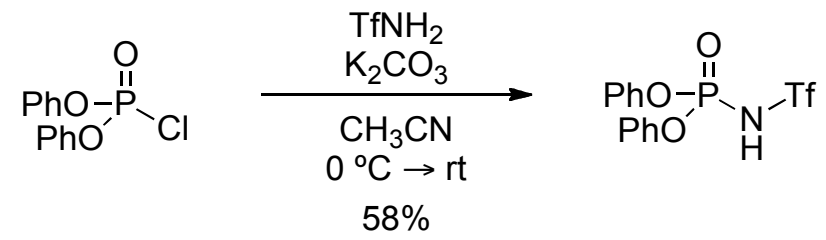

To a mixture of trifluoromethanesulfonamide $(1.31 \mathrm{~g}, 8.80 \mathrm{mmol})$ and $\mathrm{K}_{2} \mathrm{CO}_{3}(1.22 \mathrm{~g}, 8.80 \mathrm{mmol})$ in dry $\mathrm{CH}_{3} \mathrm{CN}(15 \mathrm{~mL})$ at $0{ }^{\circ} \mathrm{C}$ was slowly added diphenyl chlorophosphate $(2.0 \mathrm{~mL}, 9.68 \mathrm{mmol})$. The mixture was allowed to warm up to room temperature and was stirred for $15 \mathrm{~h}$. The reaction was quenched with water. The phases were separated and the aqueous layer was extracted with $\mathrm{Et}_{2} \mathrm{O}$. The combined organic phases were washed with saturated aqueous $\mathrm{NaHCO}_{3}$ and aqueous $\mathrm{HCl}(6 \mathrm{~N})$, respectively. After the $\mathrm{HCl}$ layer was extracted with $\mathrm{Et}_{2} \mathrm{O}$ once again, the combined organic phases were dried over $\mathrm{MgSO}_{4}$, filtered and the solvent was removed in vacuo to afford $1.95 \mathrm{~g} \mathrm{(98 \% )} \mathrm{of} \mathrm{the}$ expected pure $N$-Tf-phosphoramide as a white solid.

${ }^{1} \mathrm{H}$ NMR $\left(300 \mathrm{MHz}, \mathrm{CDCl}_{3}\right): \delta 9.21(\mathrm{br}, 1 \mathrm{H}), 7.35-7.30(\mathrm{~m}, 4 \mathrm{H}), 7.25-7.17(\mathrm{~m}, 6 \mathrm{H}) ;{ }^{13} \mathrm{C}$ NMR $(75$ $\left.\mathrm{MHz}, \mathrm{CDCl}_{3}\right): \delta 149.52\left(\mathrm{~d}, J_{\mathrm{C}-\mathrm{P}}=8.0 \mathrm{~Hz}\right), 129.99\left(\mathrm{~d}, J_{\mathrm{C}-\mathrm{P}}=0.6 \mathrm{~Hz}\right), 126.28\left(\mathrm{~d}, J_{\mathrm{C}-\mathrm{P}}=1.2 \mathrm{~Hz}\right)$, $120.06\left(\mathrm{~d}, J_{\mathrm{C}-\mathrm{P}}=4.9 \mathrm{~Hz}\right), 119.10\left(\mathrm{q}, J_{\mathrm{C}-\mathrm{F}}=319.1 \mathrm{~Hz}\right) ;{ }^{31} \mathrm{P} \mathrm{NMR}\left(243 \mathrm{MHz}, \mathrm{CDCl}_{3}\right): \delta-15.7 ;{ }^{19} \mathrm{~F}$ $\operatorname{NMR}\left(565 \mathrm{MHz}, \mathrm{CDCl}_{3}\right.$ ): $\delta-75.3$; IR (neat, $\mathrm{cm}^{-1}$ ): 2945, 2698, 1590, 1490, 1436, 1375, 1203, 1014 , 947, 754, 688; Mp: $67{ }^{\circ} \mathrm{C}$; HRMS (FAB, matrix: 3-nitrobenzyl alcohol, positive): $\mathrm{m} / \mathrm{z}$ calcd for $\mathrm{C}_{13} \mathrm{H}_{12} \mathrm{~F}_{3} \mathrm{NO}_{5} \mathrm{PS}[\mathrm{M}+\mathrm{H}]^{+}: 382.0126$, found: 382.0125 .

\section{2) Transannular reaction of cyclic amide 1a using phosphoramidate}<smiles>CC1=CCN([As])CC(C)=CCC1</smiles>

$(R)-1 \mathbf{a}$ $(>98 \%$ ee $)$

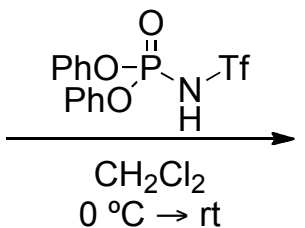

$59 \%$

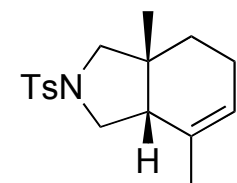
$14 a$
$(>98 \%$ dr, $>98 \%$ ee $)$

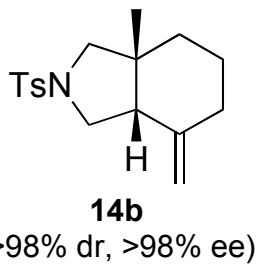

$29 \%$

To a solution of $(R)-1 \mathrm{a}(>98 \%$ ee; $20.0 \mathrm{mg}, 0.0655 \mathrm{mmol})$ in dry $\mathrm{CH}_{2} \mathrm{Cl}_{2}(5 \mathrm{~mL})$ at $0{ }^{\circ} \mathrm{C}$ was added $N$-Tf-phosphoramide $(37.5 \mathrm{mg}, 0.0982 \mathrm{mmol})$. The mixture was allowed to warm up to room temperature and was stirred for $30 \mathrm{~h}$. The reaction was quenched with saturated aqueous $\mathrm{NaHCO}_{3}$. The phases were separated and the aqueous layer was extracted with $\mathrm{CH}_{2} \mathrm{Cl}_{2}$. The combined organic phases were dried over $\mathrm{Na}_{2} \mathrm{SO}_{4}$, filtered and the solvent was removed in vacuo. The residue was purified by silica gel chromatography (hexane/AcOEt $=1 / 0$ to $12 / 1$ ) to afford $17.5 \mathrm{mg}$ of a mixture of $14 \mathbf{a}$ and $14 \mathbf{b}\left(88 \% ; \mathbf{1 4 a} / \mathbf{1 4 b}=67 / 33\right.$ determined by ${ }^{1} \mathrm{H}$ NMR analysis $)$ as a white solid. The mixture of 14a and 14b were separated by chiral HPLC; column: Daicel CHIRALPAK AS-H column $(2.0 \mathrm{~cm} \times 25 \mathrm{~cm})$, eluent: hexane/EtOH =2/1, flow rate: $4.0 \mathrm{~mL} / \mathrm{min}$, detection: UV $254 \mathrm{~nm}$, temperature: rt. Both transannular products 14a and $\mathbf{1 4 b}$ were determined as a single stereoisomer (>98\% dr analyzed by ${ }^{1} \mathrm{H}$ NMR, $>98 \%$ ee analyzed by chiral HPLC).

\section{3a,7-dimethyl-2-(p-toluenesulfonyl)-2,3,3a,4,5,7a-hexahydro- $1 H$-isoindole (14a)}

\footnotetext{
${ }^{1}$ (a) Rueping, M.; Nachtsheim, B. J.; Moreth, S. A.; Bolte, M. Angew. Chem., Int. Ed. 2008, 47, 593-596. (b) Schneekloth, Jr., J. S.; Kim, J.; Sorensen, E. J. Tetrahedron 2009, 65, 3096-3101.
} 
${ }^{1} \mathrm{H}$ NMR $\left(300 \mathrm{MHz}, \mathrm{CDCl}_{3}\right): \delta 7.70(\mathrm{~d}, J=8.1 \mathrm{~Hz}, 2 \mathrm{H}), 7.32(\mathrm{~d}, J=8.1 \mathrm{~Hz}, 2 \mathrm{H}), 5.35-532(\mathrm{~m}, 1 \mathrm{H})$, $3.67(\mathrm{dd}, J=9.6,9.0 \mathrm{~Hz}, 1 \mathrm{H}), 3.17(\mathrm{~d}, J=9.8 \mathrm{~Hz}, 1 \mathrm{H}), 3.06(\mathrm{~d}, J=9.8 \mathrm{~Hz}, 1 \mathrm{H}), 2.95(\mathrm{dd}, J=9.6$, $9.2 \mathrm{~Hz}, 1 \mathrm{H}), 2.44$ (s, 3H), $2.03(\mathrm{dd}, J=9.2,9.0 \mathrm{~Hz}, 1 \mathrm{H}), 1.98-1.83(\mathrm{~m}, 2 \mathrm{H}), 1.59(\mathrm{~d}, J=1.5 \mathrm{~Hz}, 3 \mathrm{H})$, $1.28-1.12(\mathrm{~m}, 2 \mathrm{H}), 0.92(\mathrm{~s}, 3 \mathrm{H}) ;{ }^{13} \mathrm{C} \mathrm{NMR}\left(75 \mathrm{MHz}, \mathrm{CDCl}_{3}\right): \delta 143.2,133.9,131.7,129.5,127.4$, 121.4, 60.5, 53.0, 48.7, 39.4, 28.8, 23.2, 22.6, 22.5, 21.7; IR (neat, $\mathrm{cm}^{-1}$ ): 2923, 1598, 1448, 1343, 1160, 1094, 813, 709, 666, 583; Mp: $105^{\circ} \mathrm{C}$; Analytical HPLC [column: CHIRALCEL AS-H (4.6 $\mathrm{mm} \times 25 \mathrm{~cm})$, eluent: hexane $/ \mathrm{EtOH}=2 / 1$, flow rate: $0.5 \mathrm{~mL} / \mathrm{min}$, detection: UV $254 \mathrm{~nm}$, temperature: $\mathrm{rt}] t_{R}=14.9 \mathrm{~min}$ for $(+)$-isomer, $16.5 \mathrm{~min}$ for (-)-isomer; Optical rotation value: $[\alpha]_{\mathrm{D}}{ }^{20}$ $8.77\left(c 0.85, \mathrm{CHCl}_{3}\right)$ for $(+)$-isomer ( $>98 \%$ ee); HRMS (EI, positive): $m / z$ calcd for $\mathrm{C}_{17} \mathrm{H}_{23} \mathrm{NO}_{2} \mathrm{~S}$ $[\mathrm{M}]^{+}:$305.1449, found: 305.1445.

\section{3a-methyl-7-methylene-2-(p-toluenesulfonyl)octahydroisoindole (14b)}

${ }^{1} \mathrm{H}$ NMR $\left(300 \mathrm{MHz} \mathrm{CDCl}_{3}\right): \delta 7.72(\mathrm{~d}, J=8.3 \mathrm{~Hz}, 2 \mathrm{H}), 7.33(\mathrm{~d}, J=8.3 \mathrm{~Hz}, 2 \mathrm{H}), 4.77$ (s, $\left.1 \mathrm{H}\right), 4.68$ (s, 1H), $3.37(\mathrm{dd}, J=10.4,10.1 \mathrm{~Hz}, 1 \mathrm{H}), 3.33(\mathrm{dd}, J=10.4,9.6 \mathrm{~Hz}, 1 \mathrm{H}), 3.26(\mathrm{~d}, J=9.5 \mathrm{~Hz}, 1 \mathrm{H})$, $2.91(\mathrm{~d}, J=9.5 \mathrm{~Hz}, 1 \mathrm{H}), 2.45(\mathrm{~s}, 3 \mathrm{H}), 2.33(\mathrm{dd}, J=10.1,9.6 \mathrm{~Hz}, 1 \mathrm{H}), 2.15-1.96(\mathrm{~m}, 2 \mathrm{H}), 1.63-1.52$ $(\mathrm{m}, 1 \mathrm{H}), 1.46-1.22(\mathrm{~m}, 3 \mathrm{H}), 0.92(\mathrm{~s}, 3 \mathrm{H}) ;{ }^{13} \mathrm{C} \mathrm{NMR}$ (75 MHz, $\left.\mathrm{CDCl}_{3}\right): \delta 143.7,143.2,134.4,129.6$, 127.3, 112.4, 61.1, 52.6, 50.3, 42.5, 31.6, 30.3, 23.4, 21.78, 21.75; IR (neat, $\mathrm{cm}^{-1}$ ): 2933, 1344, 1159, 1094, 810, 665, 600, 544; Mp: $76{ }^{\circ} \mathrm{C}$; Analytical HPLC [column: CHIRALCEL AS-H $(4.6 \mathrm{~mm} \times 25$ $\mathrm{cm}$ ), eluent: hexane $/ \mathrm{EtOH}=2 / 1$, flow rate: $0.5 \mathrm{~mL} / \mathrm{min}$, detection: $\mathrm{UV} 254 \mathrm{~nm}$, temperature: $\mathrm{rt}] t_{R}=$ $17.6 \mathrm{~min}$ for (+)-isomer, $22.1 \mathrm{~min}$ for (-)-isomer; Optical rotation value: $[\alpha]_{\mathrm{D}}{ }^{20}-32.8(c 0.39$, $\mathrm{CHCl}_{3}$ ) for (-)-isomer ( $>98 \%$ ee); HRMS (EI, positive): $m / z$ calcd for $\mathrm{C}_{17} \mathrm{H}_{23} \mathrm{NO}_{2} \mathrm{~S}[\mathrm{M}]^{+}: 305.1449$, found: 305.1448; Anal. calcd for $\mathrm{C}_{17} \mathrm{H}_{23} \mathrm{NO}_{2} \mathrm{~S}$ : C, 66.85; H, 7.59; N, 4.59. Found: C, 66.88; H, 7.55; $\mathrm{N}, 4.54$.

Recrystallization from hexane/ $\mathrm{CH}_{2} \mathrm{Cl}_{2}$ afforded a single crystal that was suitable for X-ray analysis.

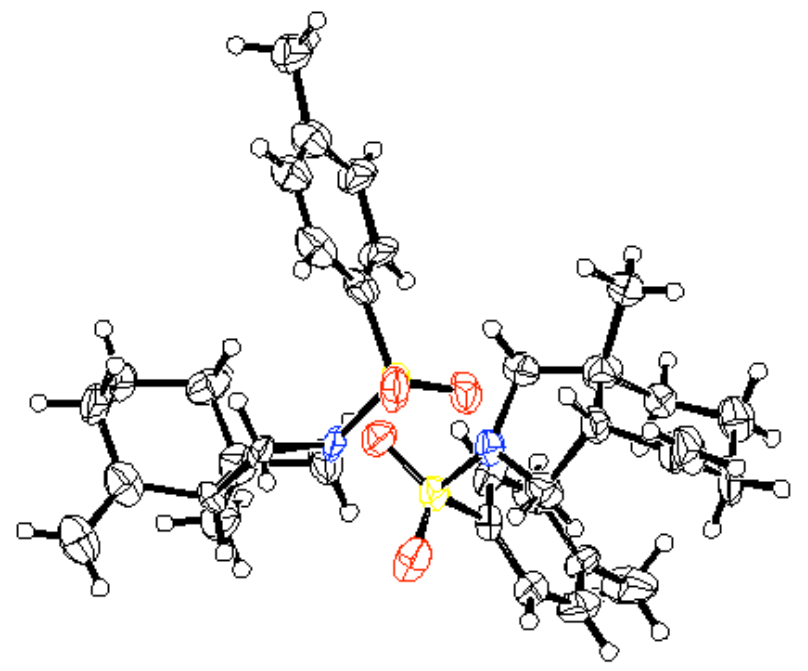

Figure S9. Molecular structure of (-)-14b (CCDC-817448) in the solid state. Thermal ellipsoids are drawn at the $50 \%$ probability level. 
3. ${ }^{1} \mathrm{H},{ }^{13} \mathrm{C},{ }^{19} \mathrm{~F}$ and ${ }^{31} \mathrm{P}$ NMR Spectra

${ }^{1}$ H NMR Chart of $\mathbf{2 a}$

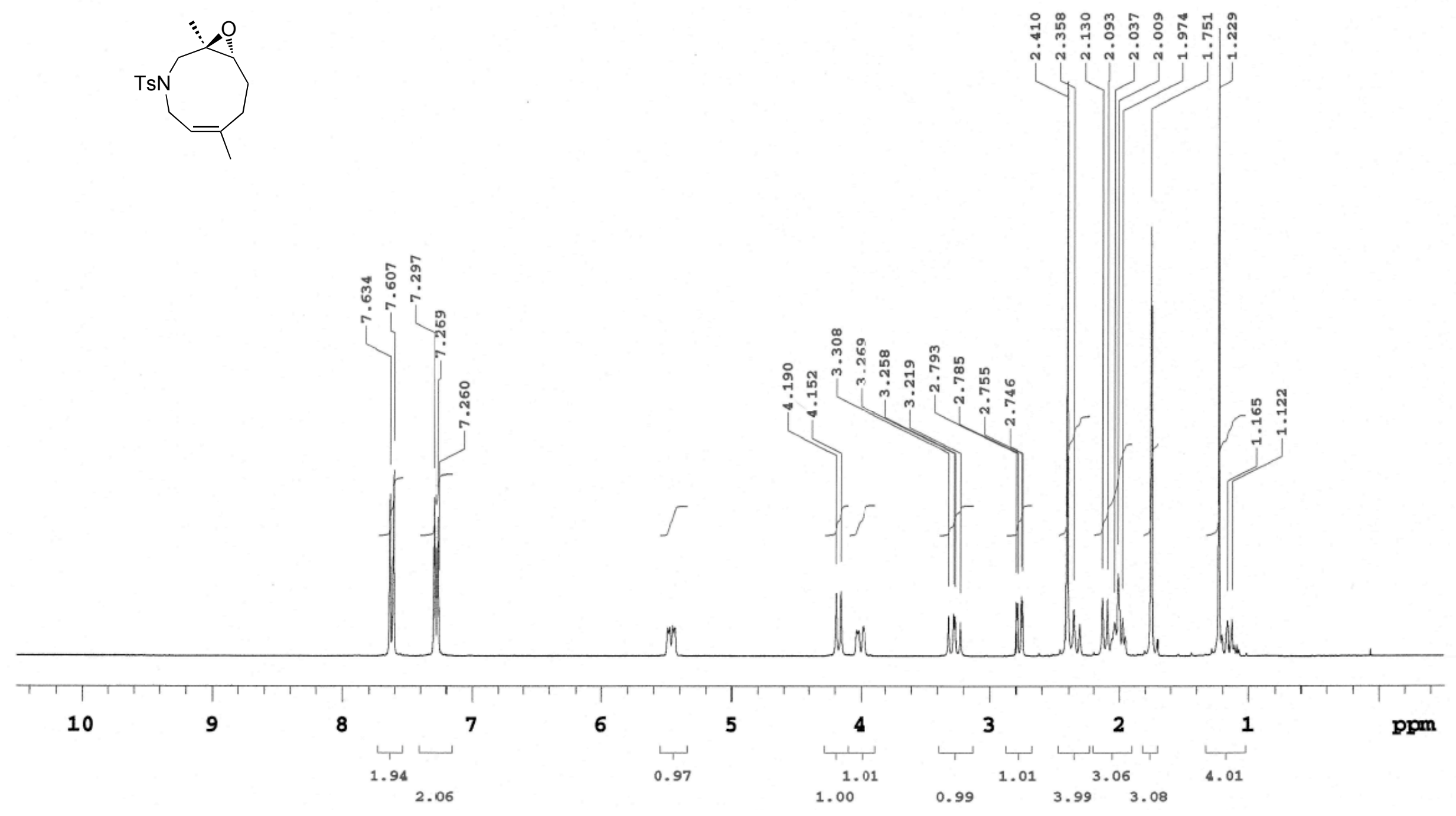


${ }^{13}$ C NMR Chart of 2a

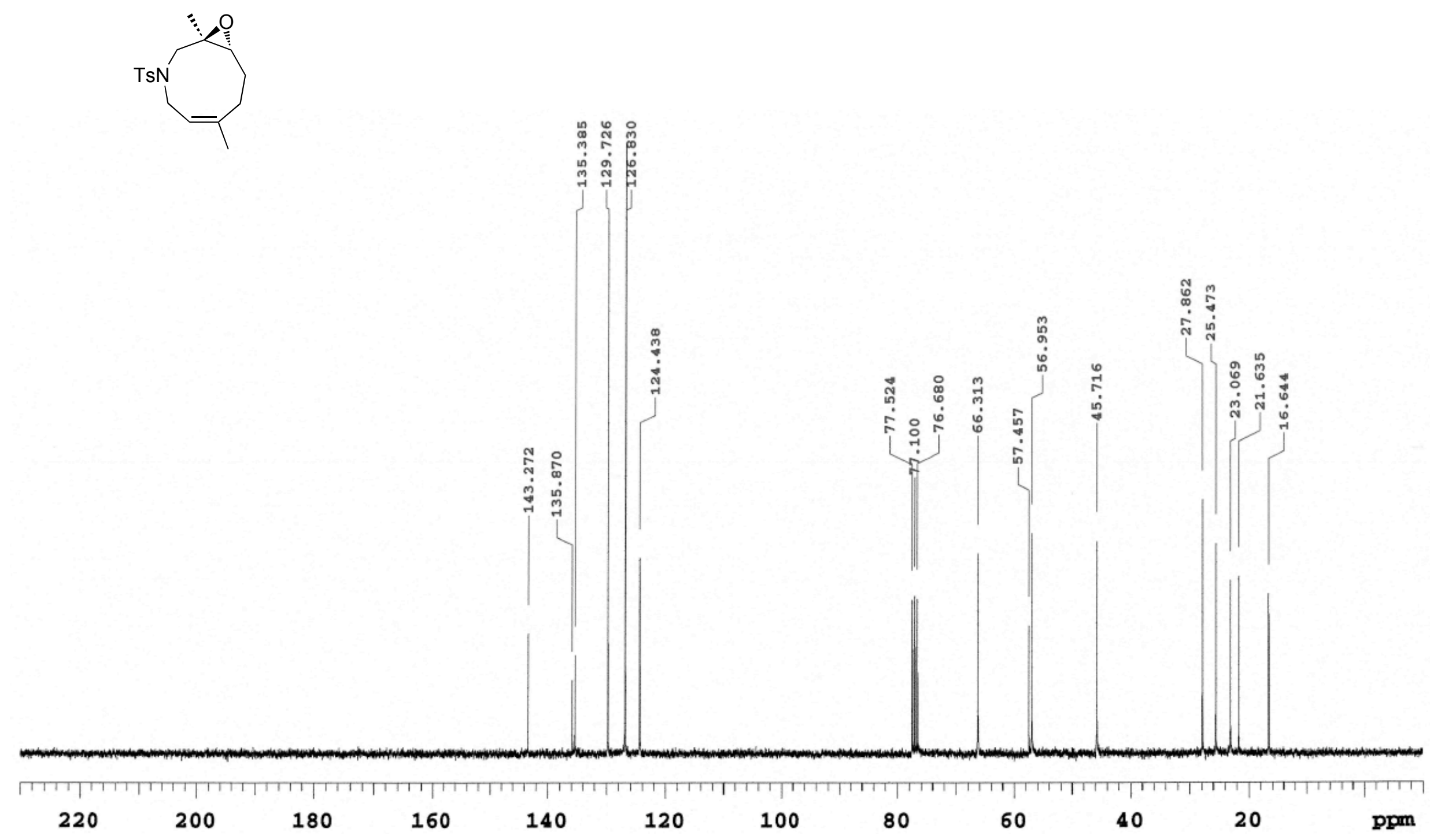




\section{${ }^{1}$ H NMR Chart of $\mathbf{3 a}$}<smiles>C/C1=C\CC[C@@]2(C)O[C@H]2CN([13CH3])C1</smiles>

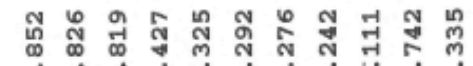

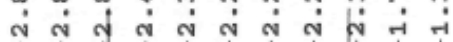
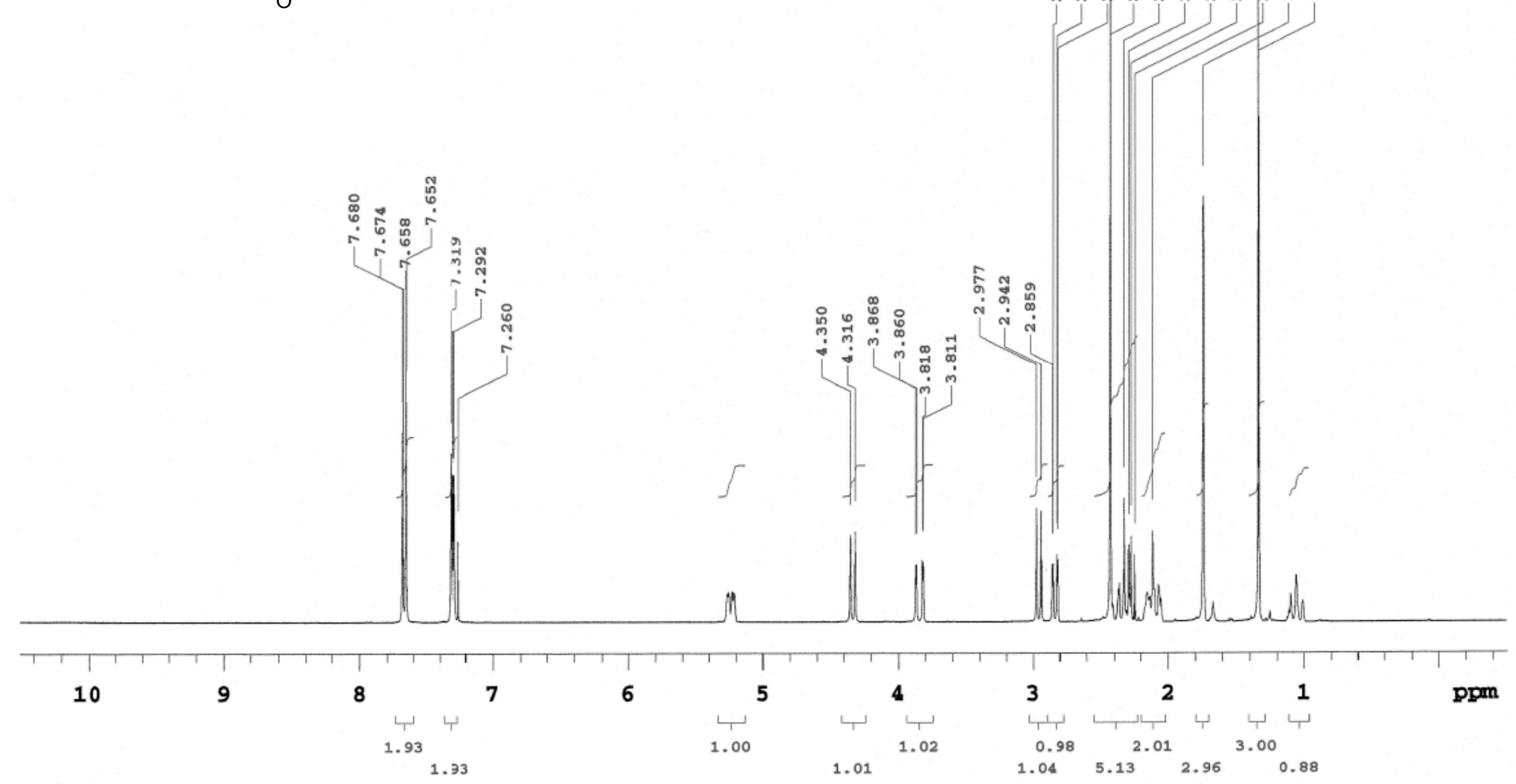
${ }^{13}$ C NMR Chart of $\mathbf{3 a}$<smiles></smiles>

응 。ำ

สิ

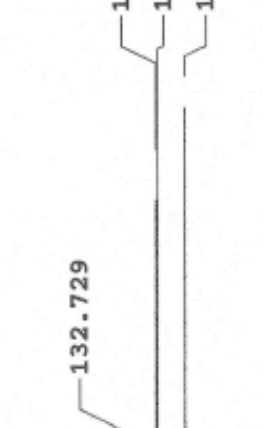


${ }^{1}$ H NMR Chart of $\mathbf{4 a}$

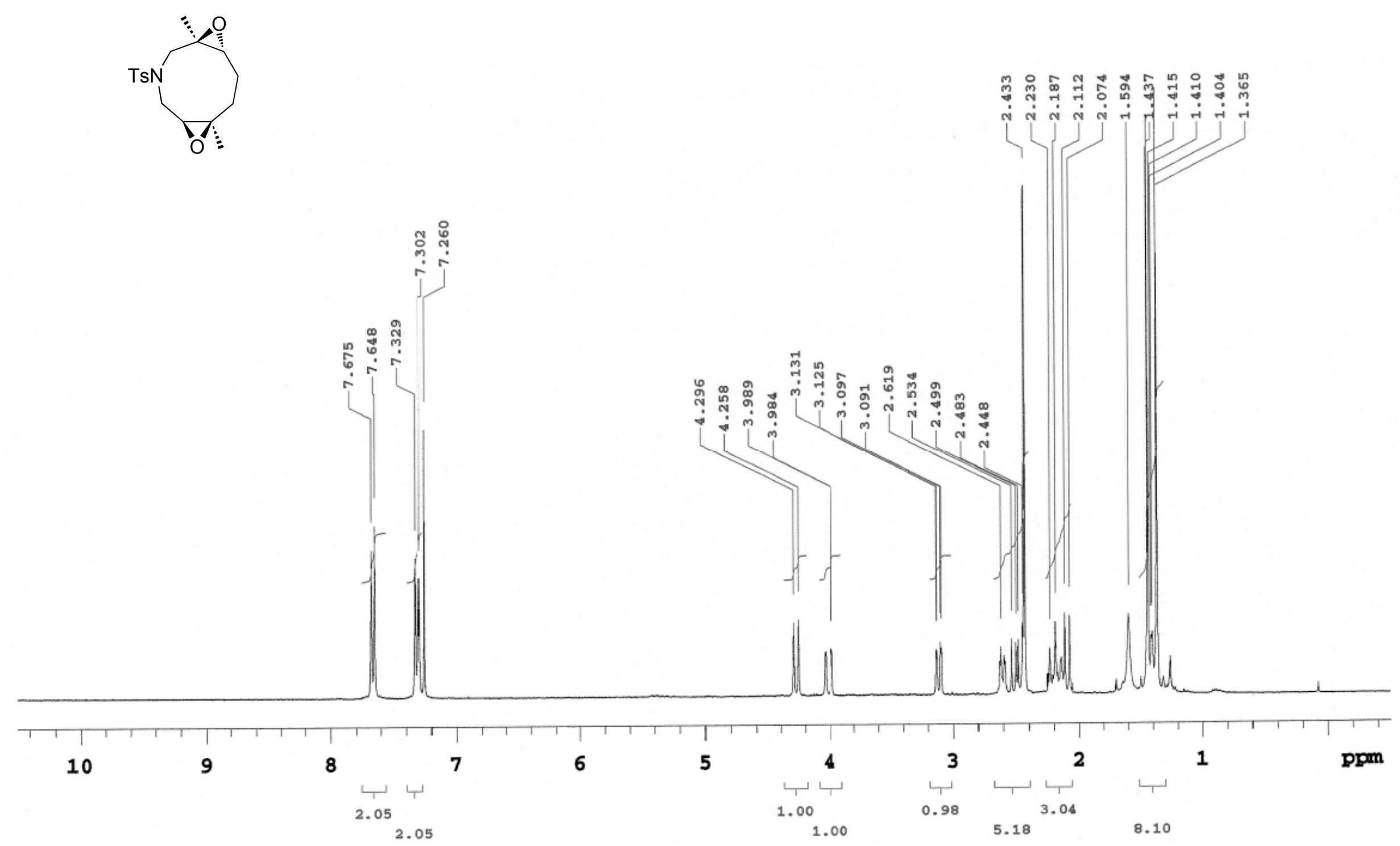


${ }^{13} \mathrm{C}$ NMR Chart of $\mathbf{4 a}$

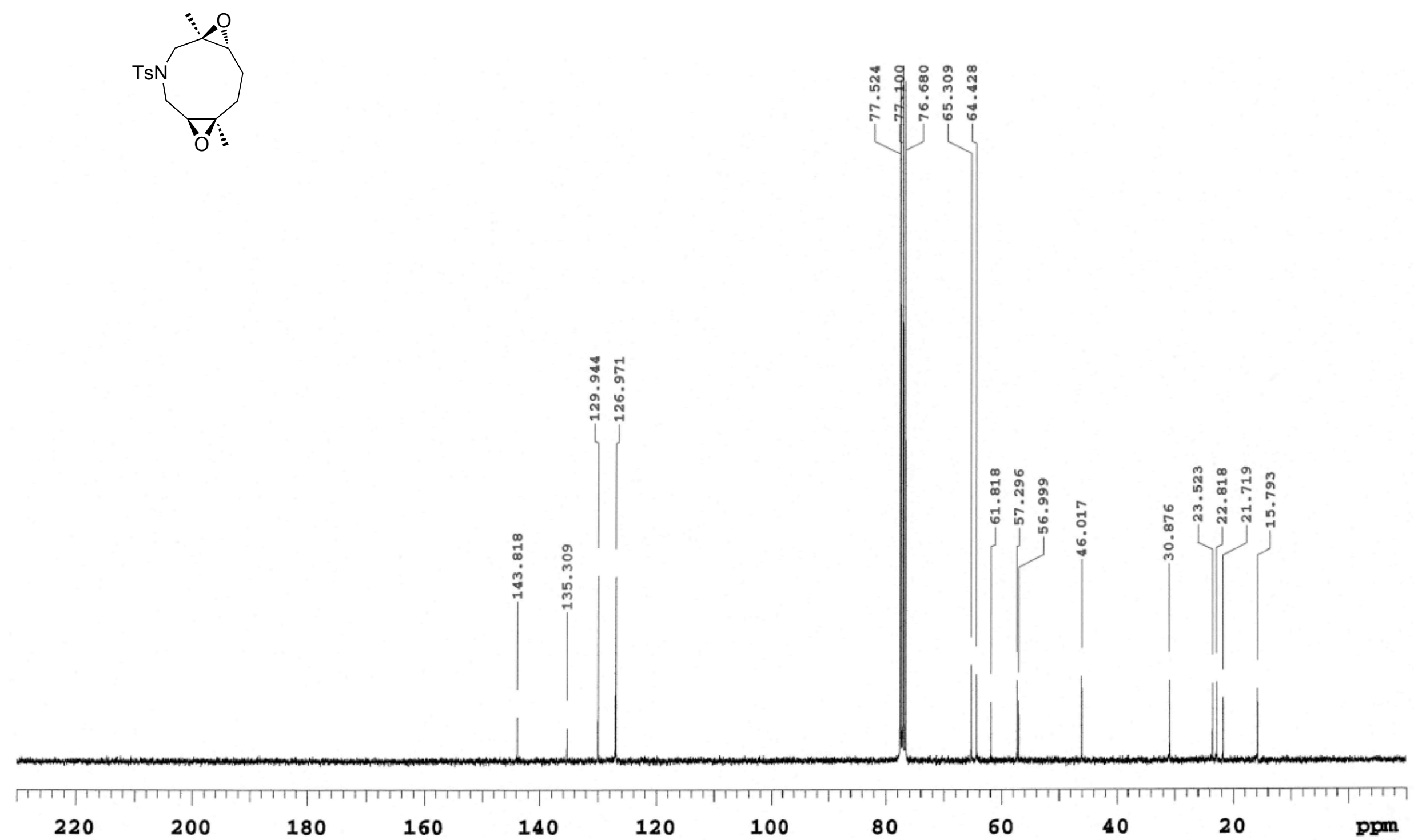


${ }^{1} \mathrm{H}$ NMR Chart of $\mathbf{2 b}$

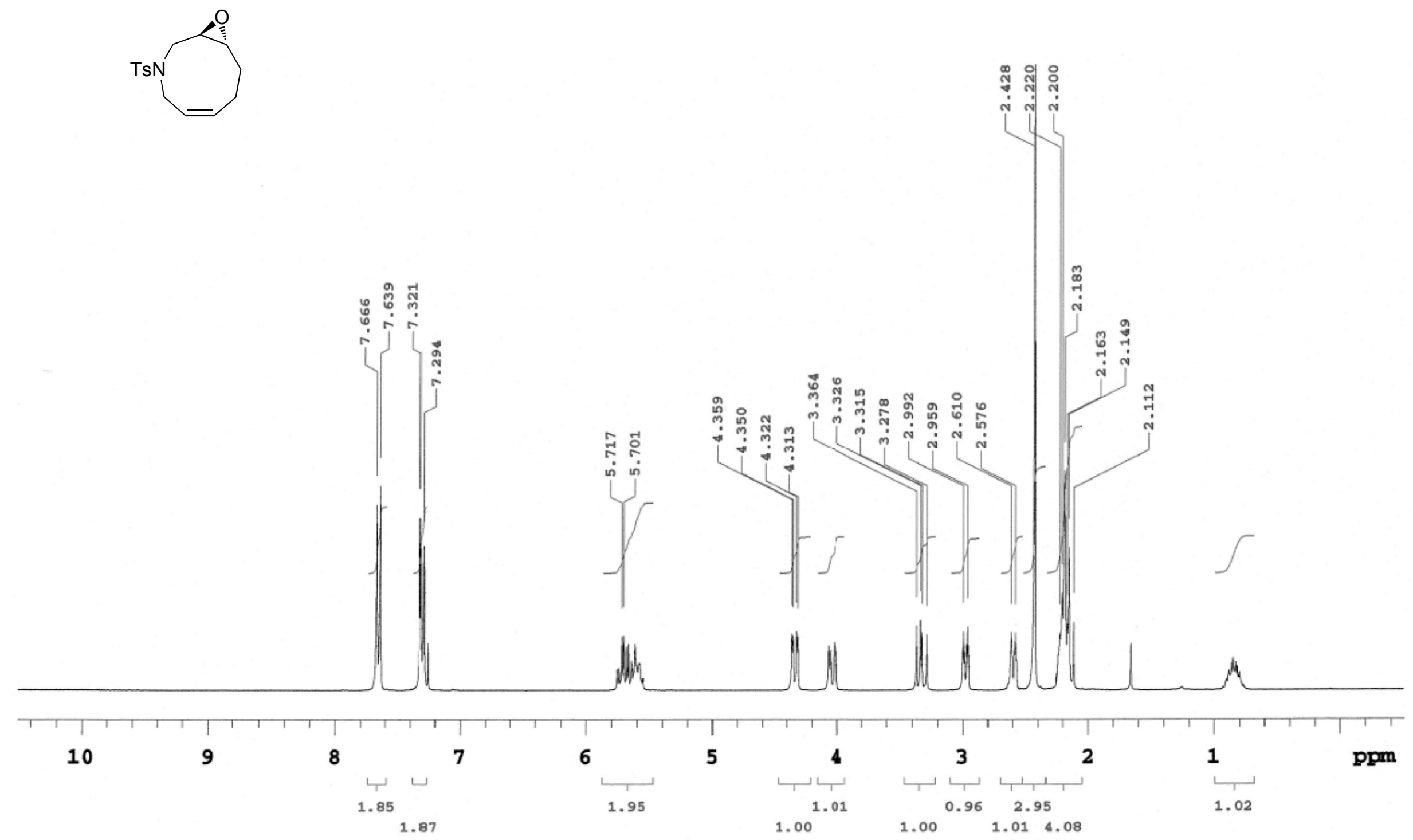


${ }^{13} \mathrm{C}$ NMR Chart of $\mathbf{2 b}$

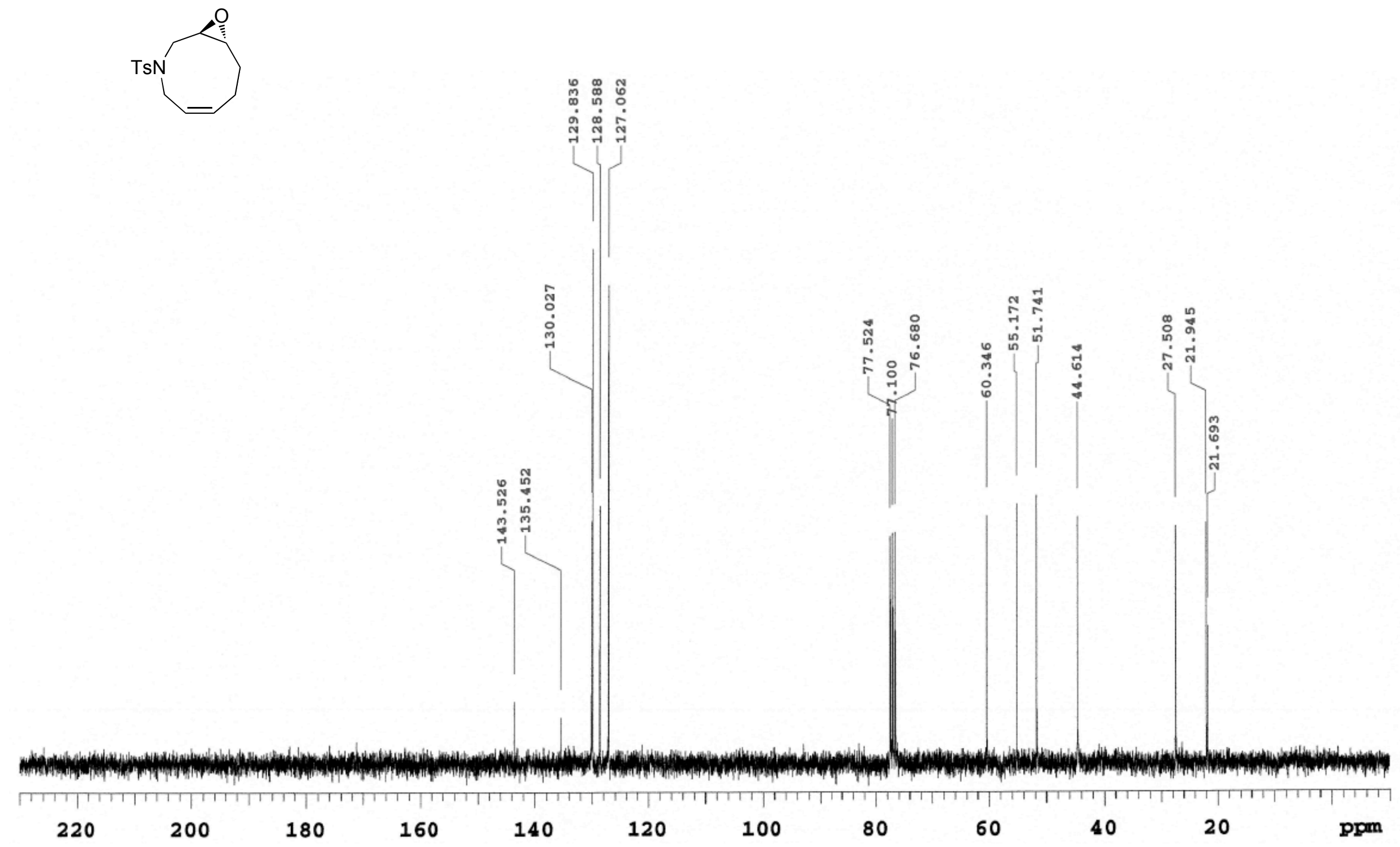


${ }^{1} \mathrm{H}$ NMR Chart of $\mathbf{3 b}$<smiles>[Y5]N1C/C=C/CCC2OC2C1</smiles>

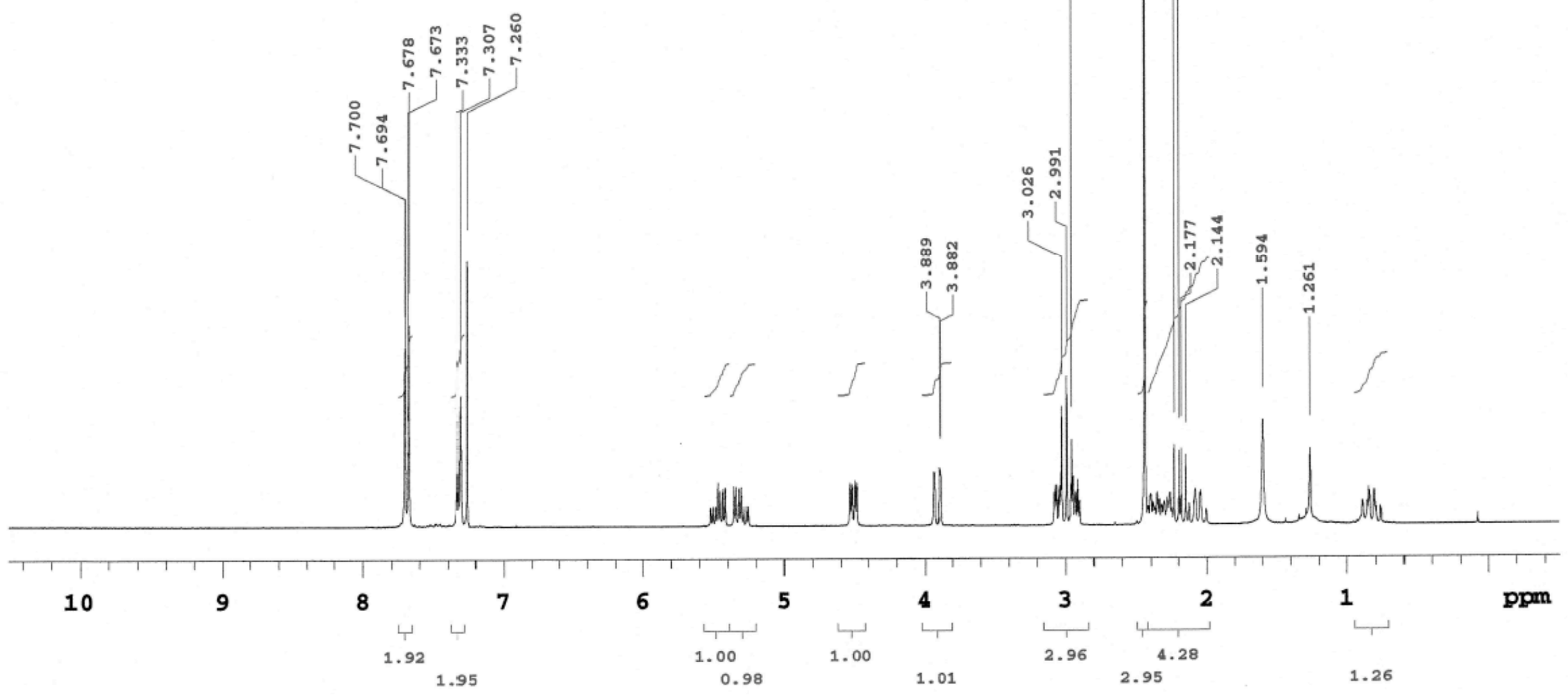


${ }^{13} \mathrm{C}$ NMR Chart of $\mathbf{3 b}$<smiles>[3H]N1C/C=C/CCC2OC2C1</smiles>

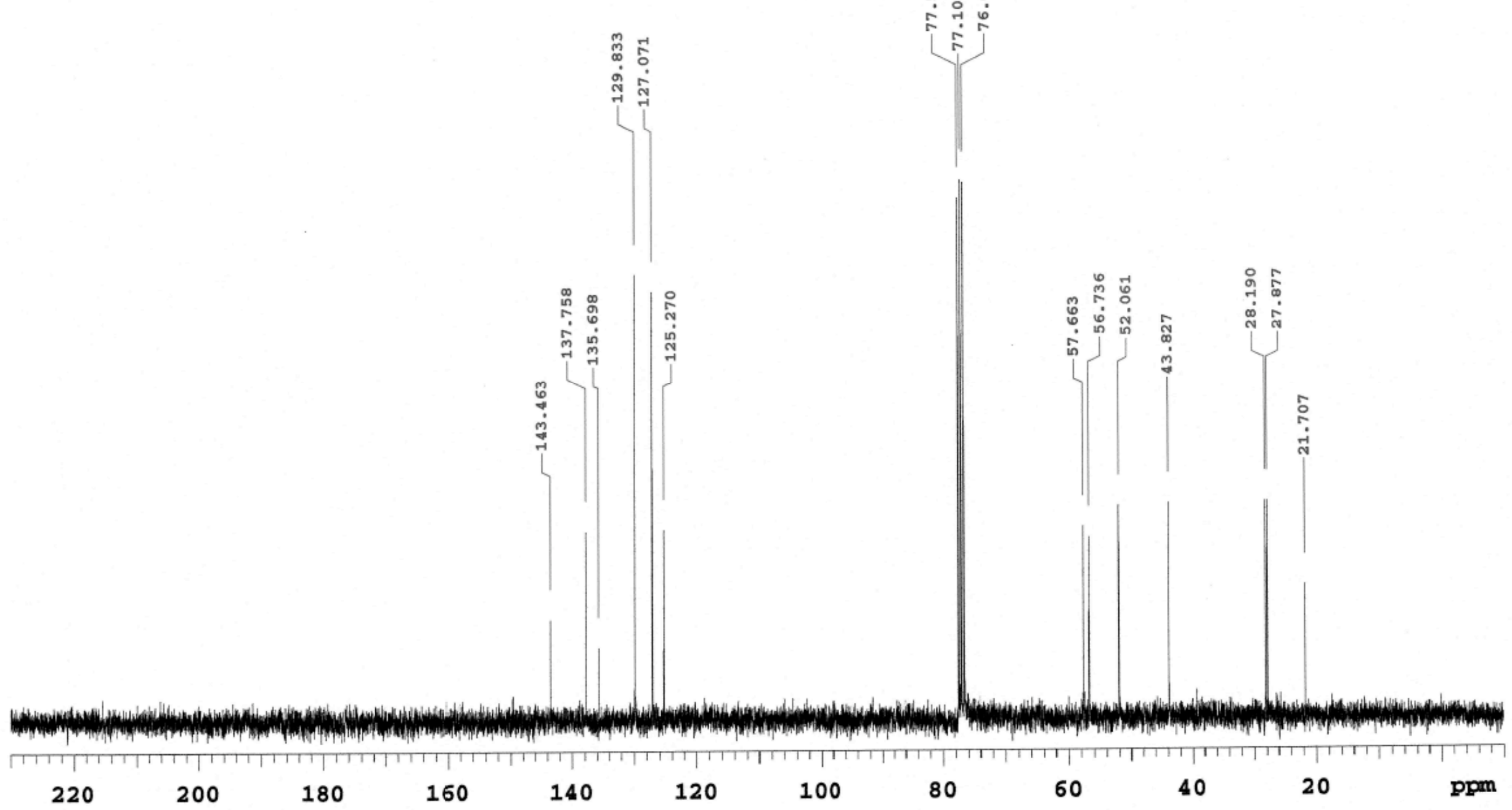


${ }^{1} \mathrm{H}$ NMR Chart of $\mathbf{4 b}$

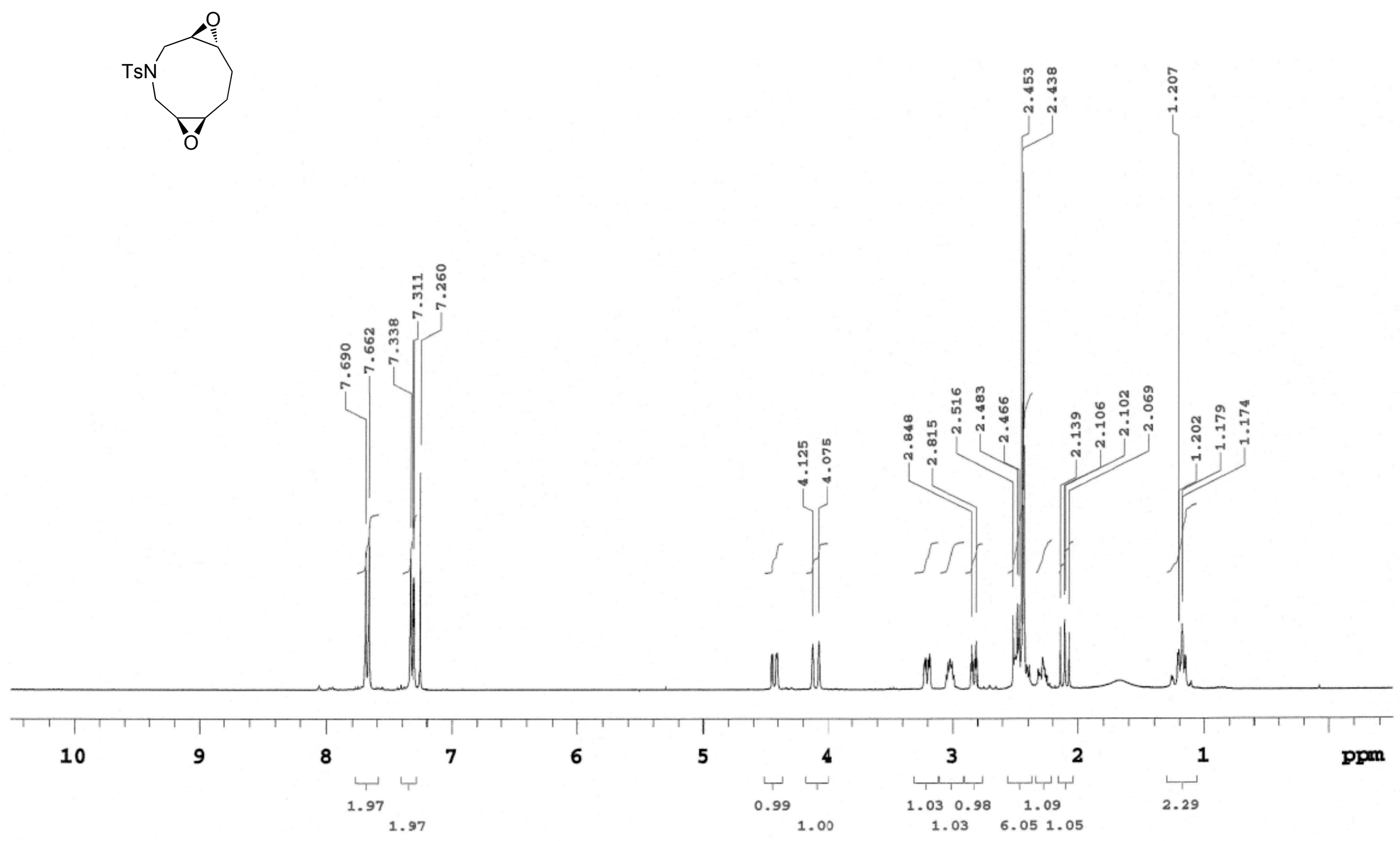


${ }^{13} \mathrm{C}$ NMR Chart of $\mathbf{4 b}$

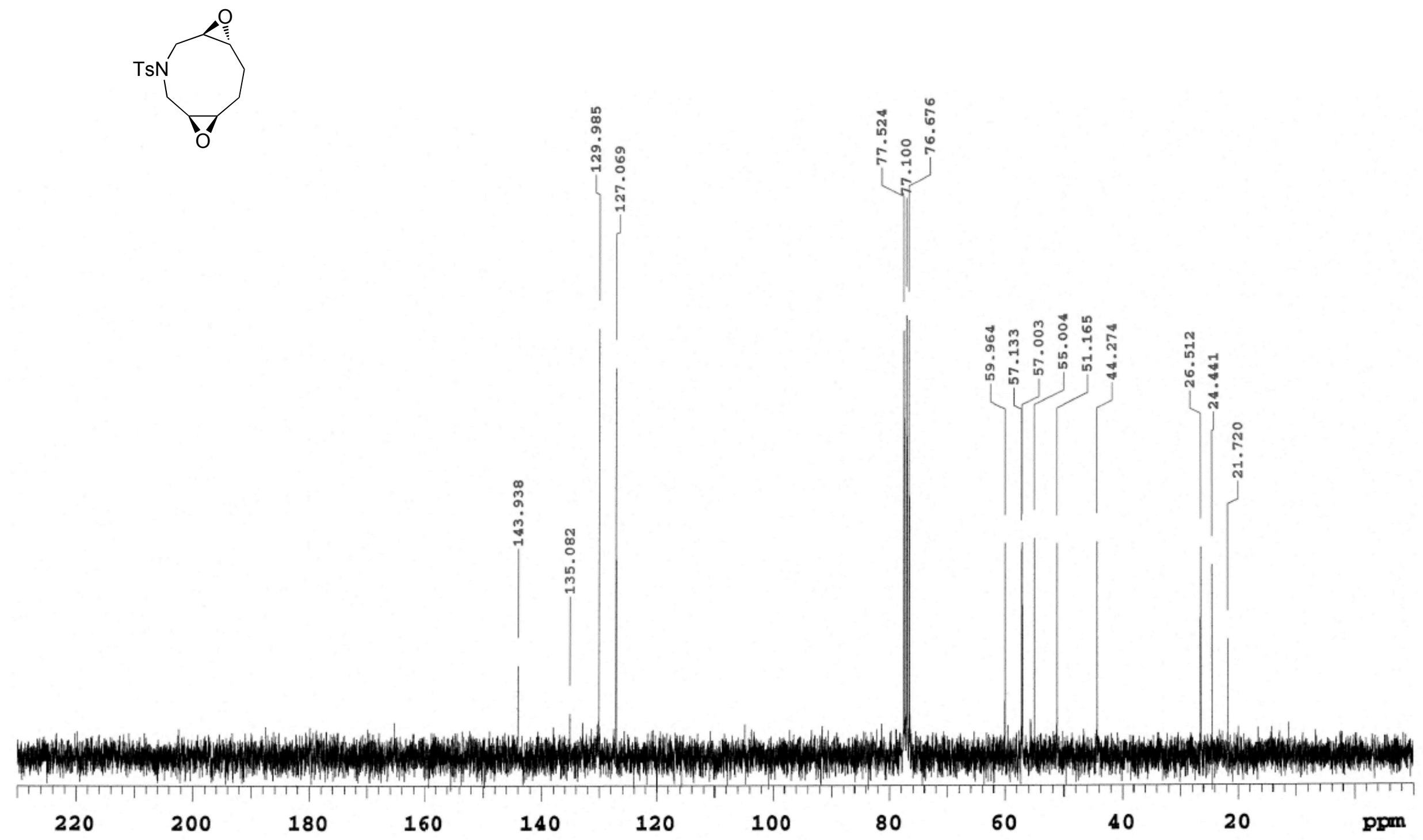


${ }^{1}$ H NMR Chart of $\mathbf{5 a}$

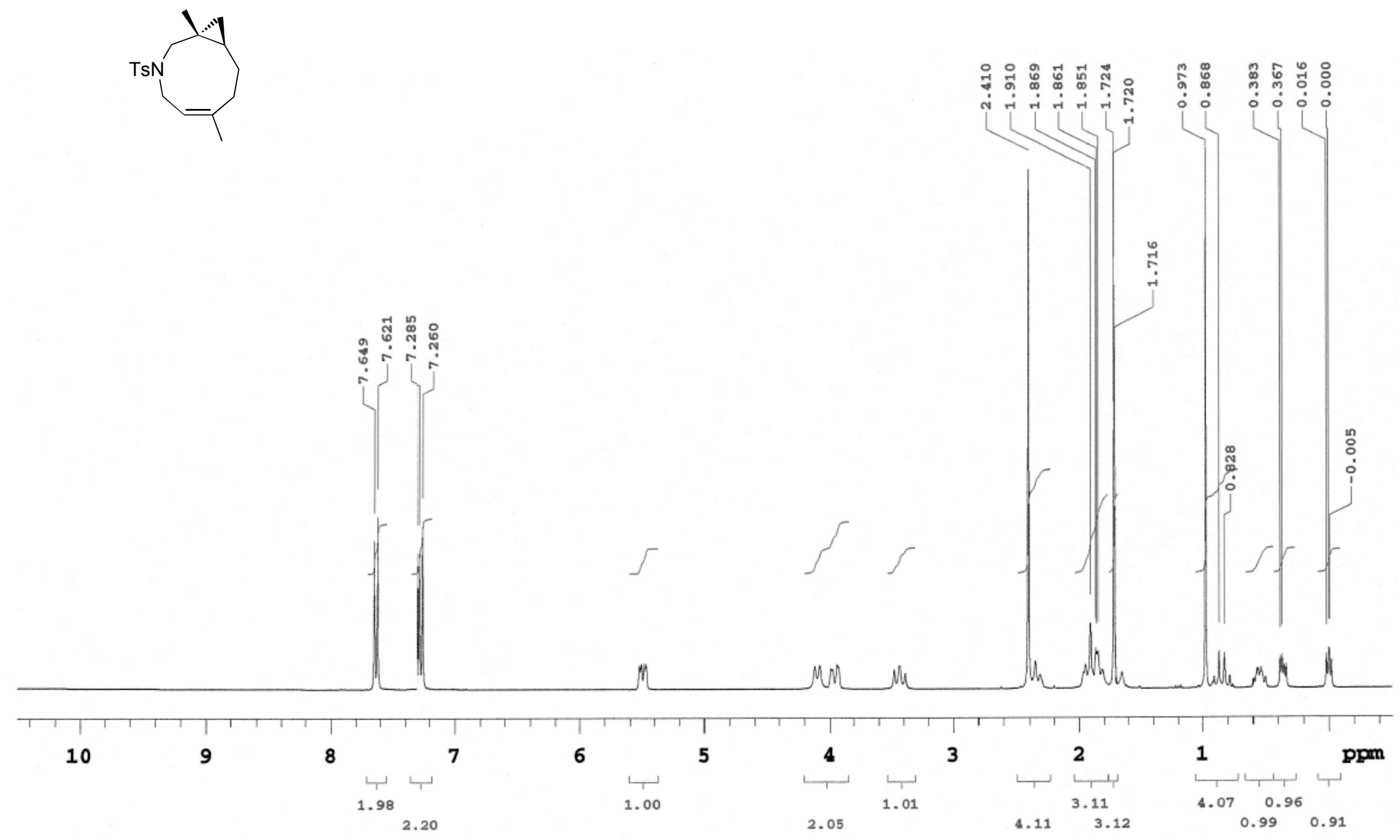


${ }^{13}$ C NMR Chart of $5 \mathbf{a}$

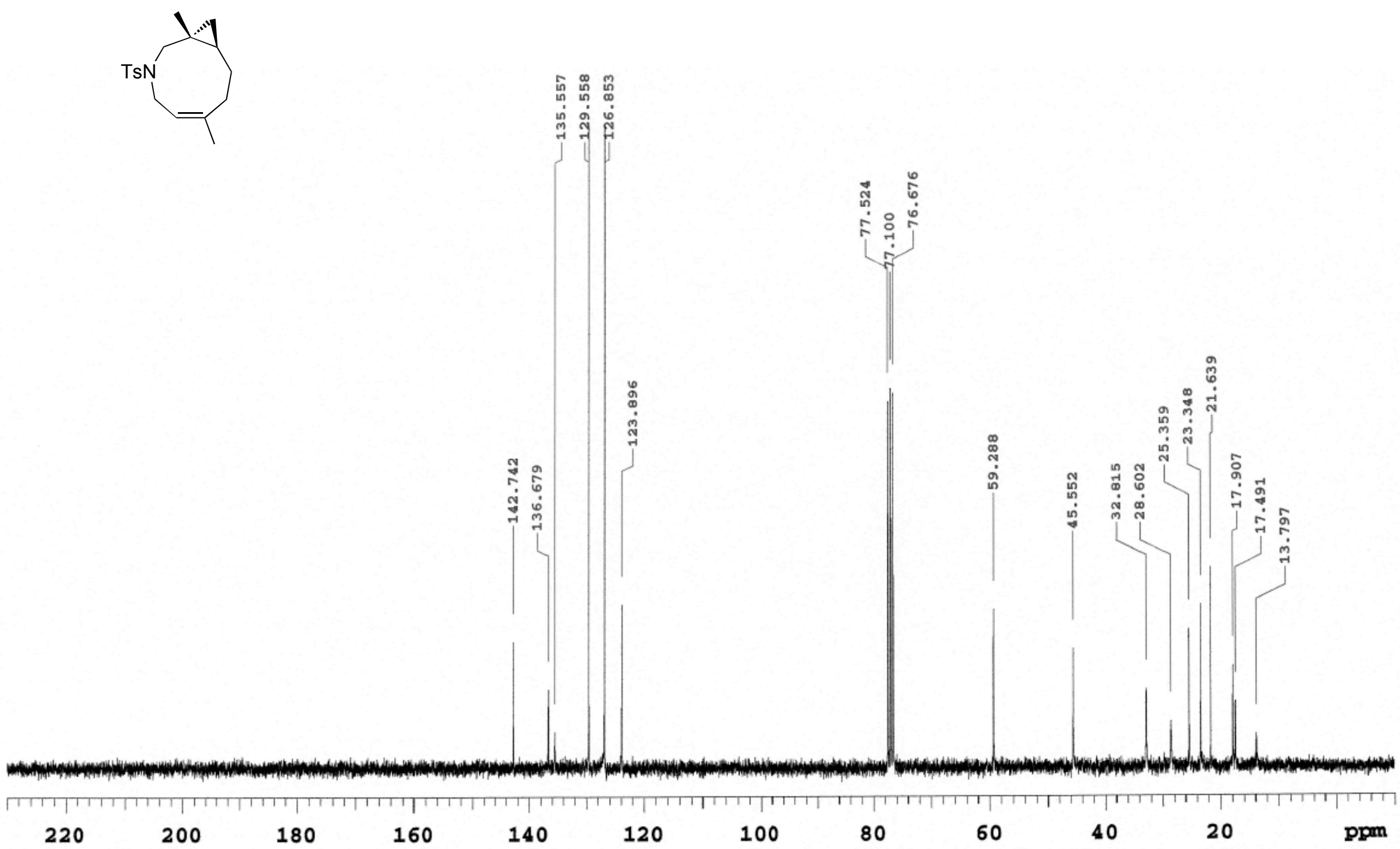


${ }^{1}$ H NMR Chart of $6 a$

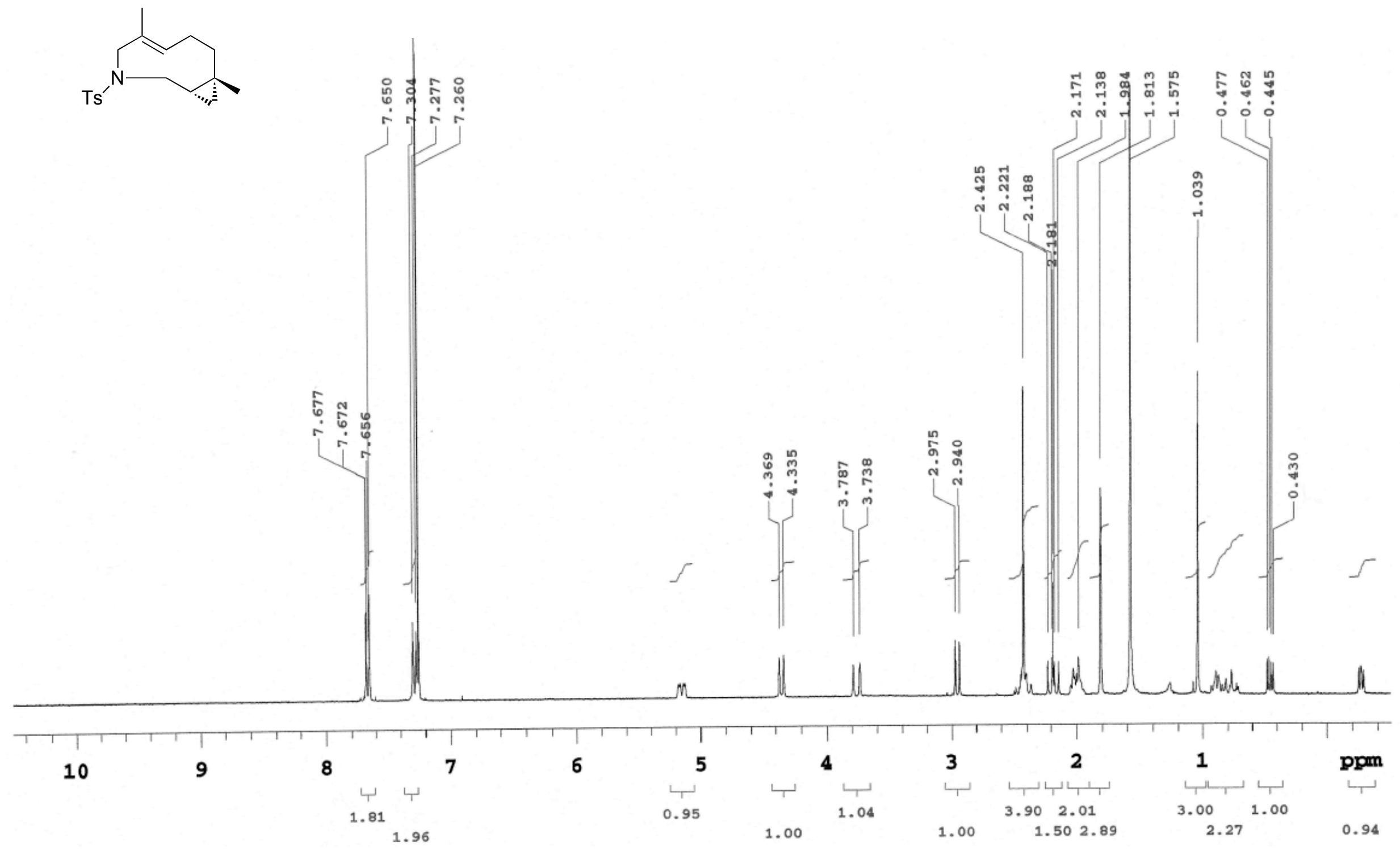


${ }^{13}$ C NMR Chart of $6 \mathbf{a}$

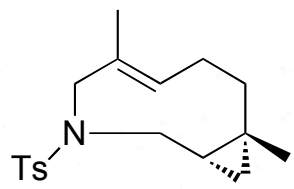

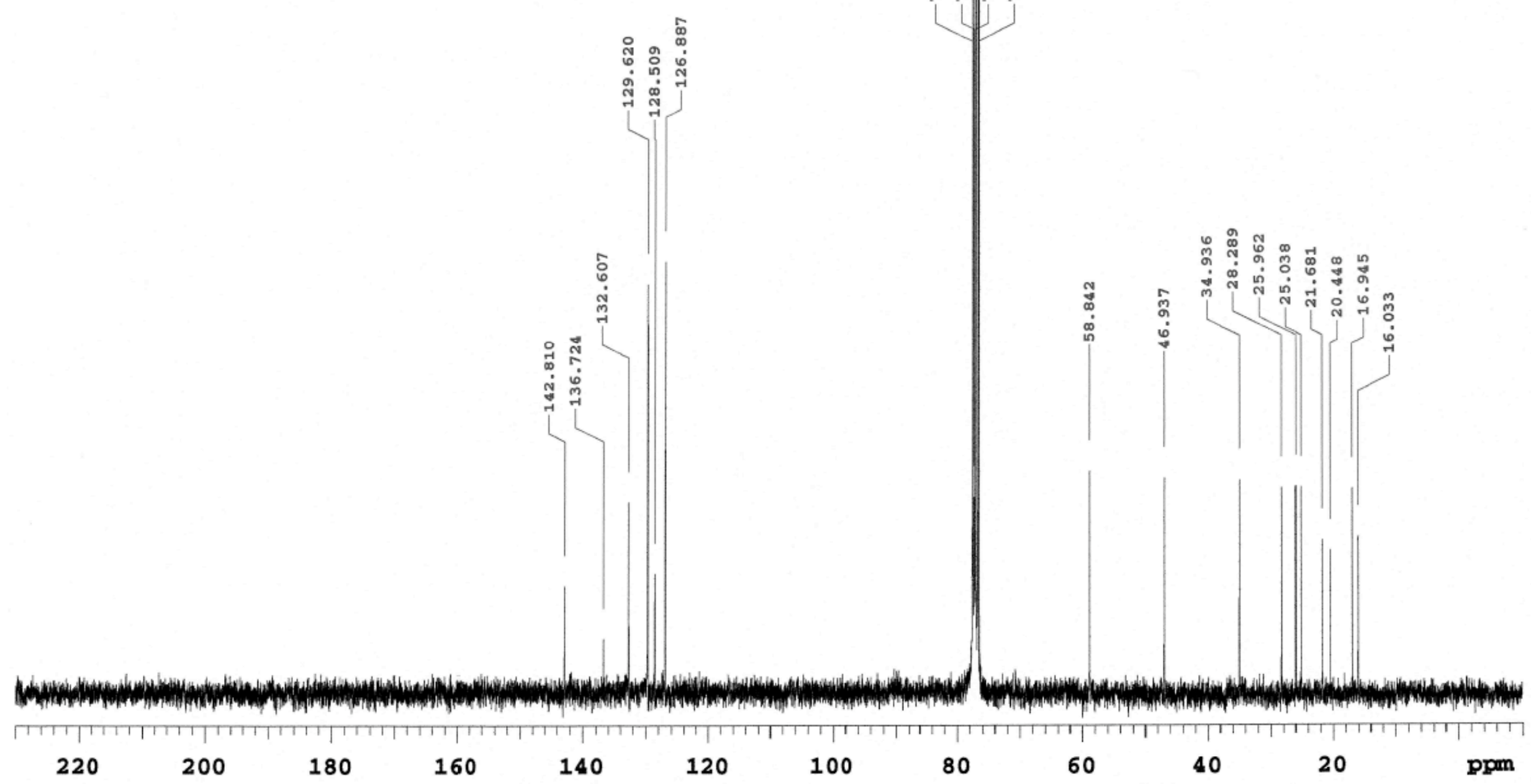


${ }^{1} \mathrm{H}$ NMR Chart of $7 \mathbf{a}$

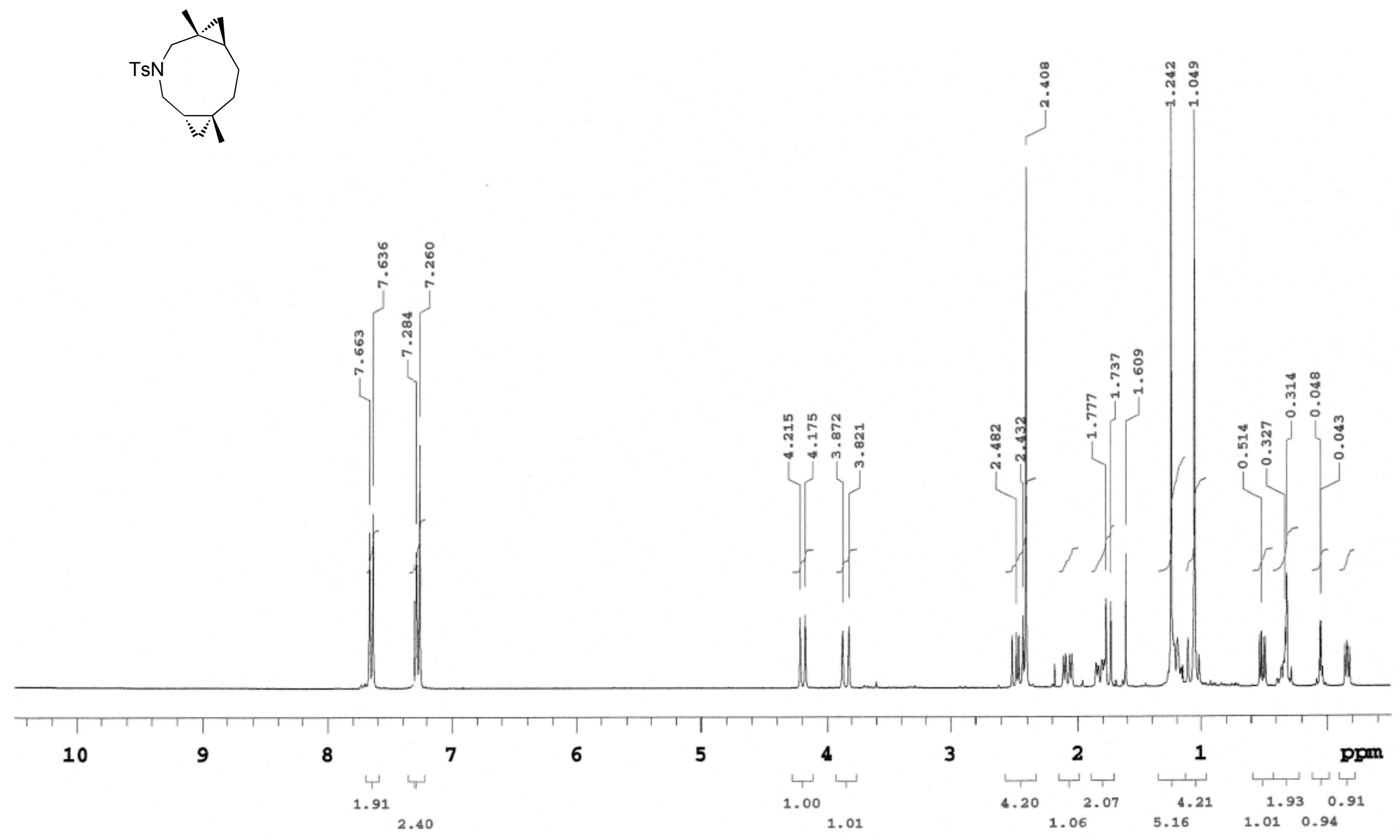


${ }^{13}$ C NMR Chart of $7 \mathbf{a}$

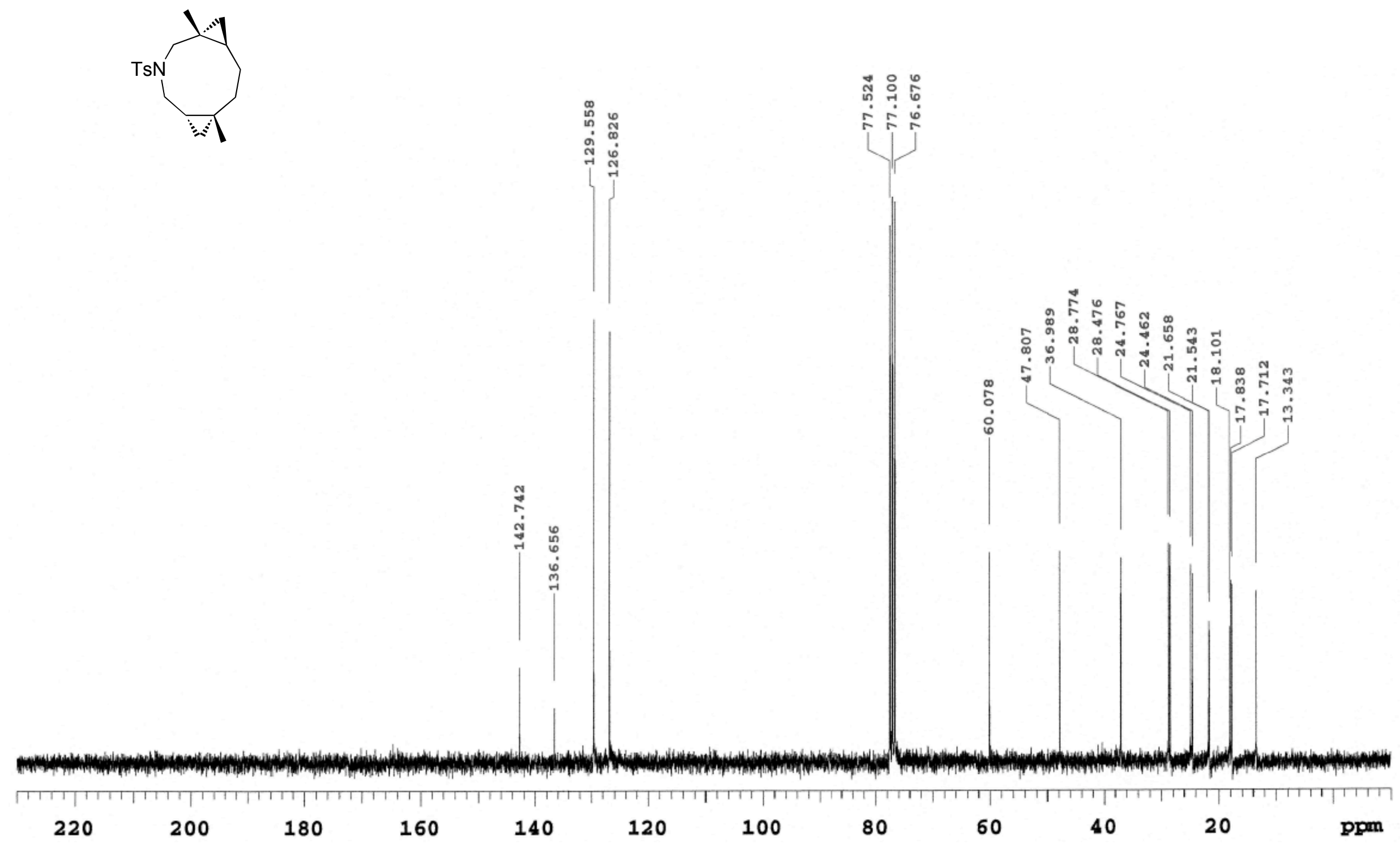


${ }^{1} \mathrm{H}$ NMR Chart of $\mathbf{5 b}$

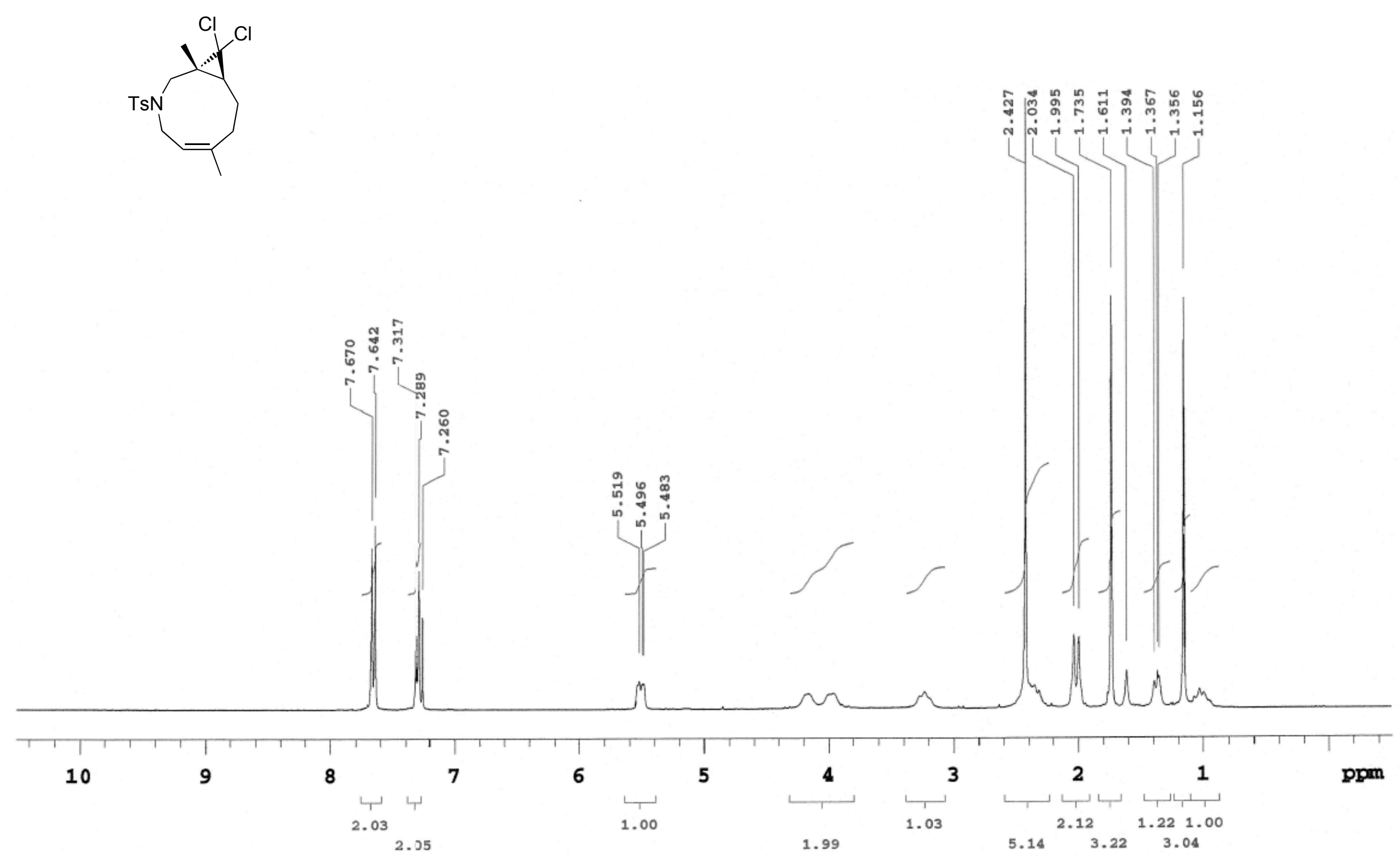


${ }^{13}$ C NMR Chart of $\mathbf{5 b}$

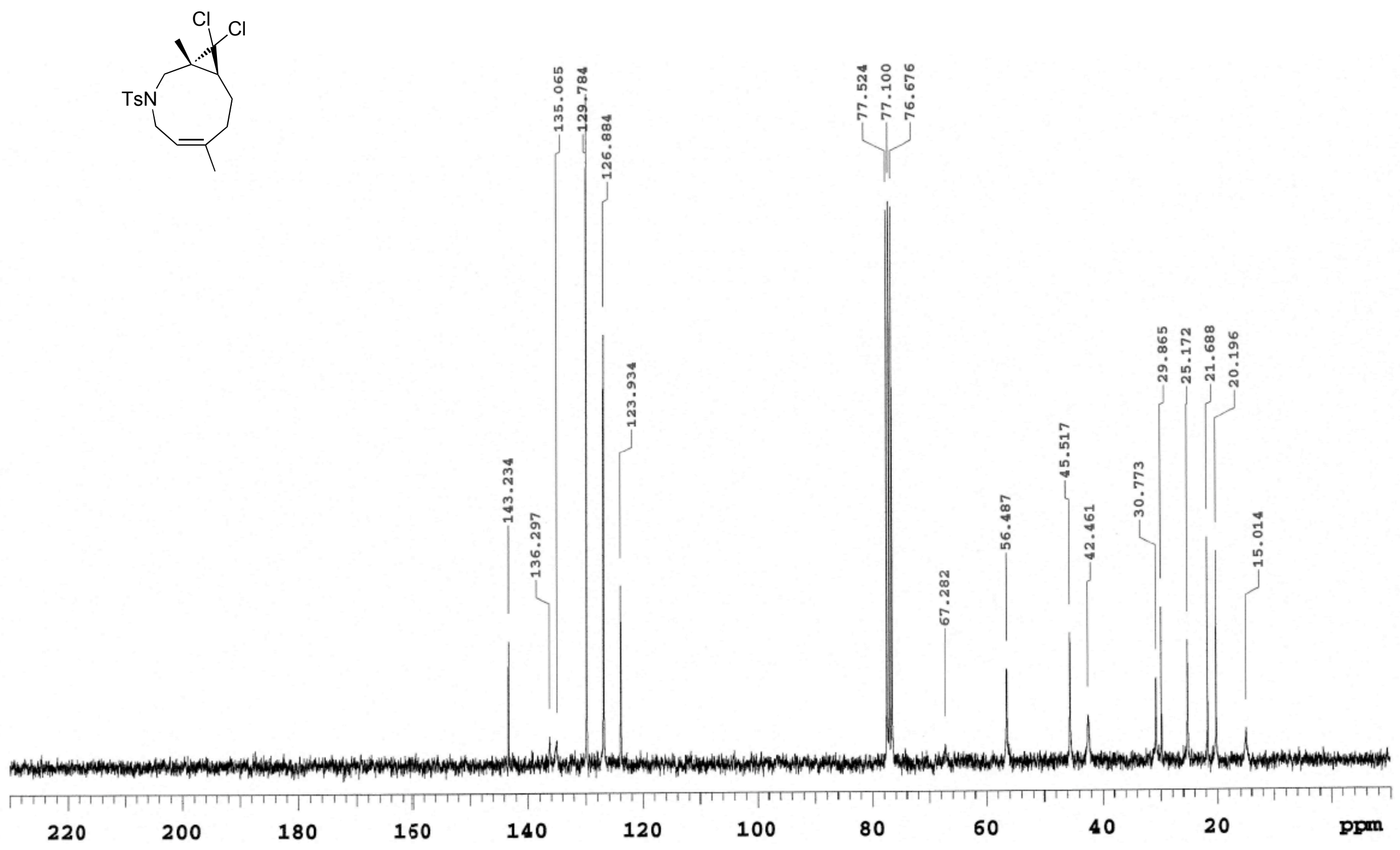


${ }^{1}$ H NMR Chart of $\mathbf{6 b}$

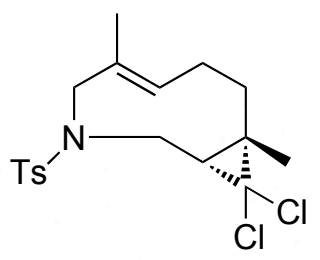

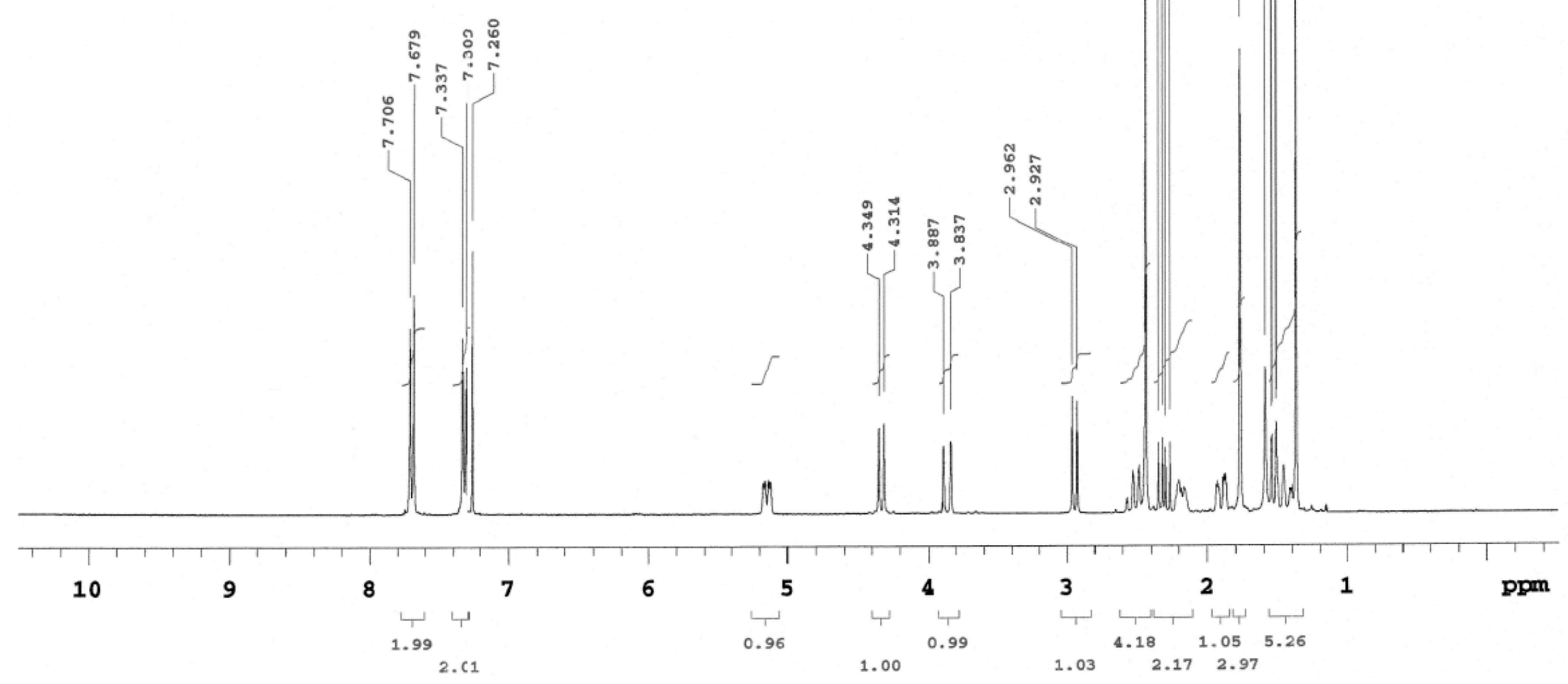


${ }^{13}$ C NMR Chart of $\mathbf{6 b}$

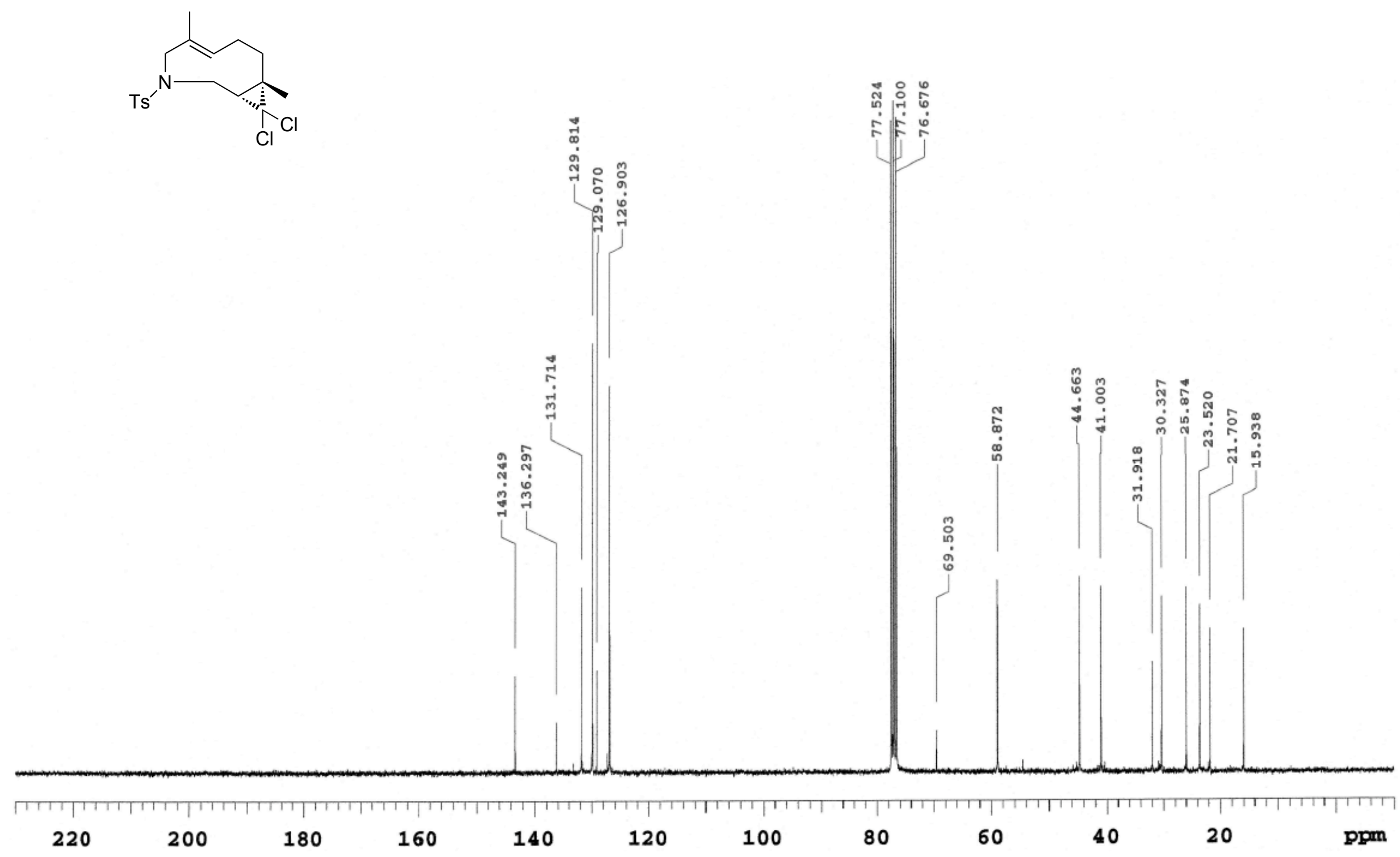


${ }^{1}$ H NMR Chart of $7 \mathbf{b}$

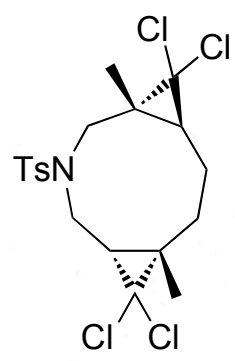

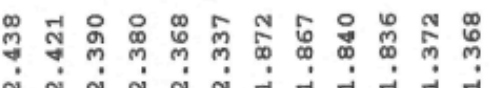

ผ ผ

ㄷ. 유ำ
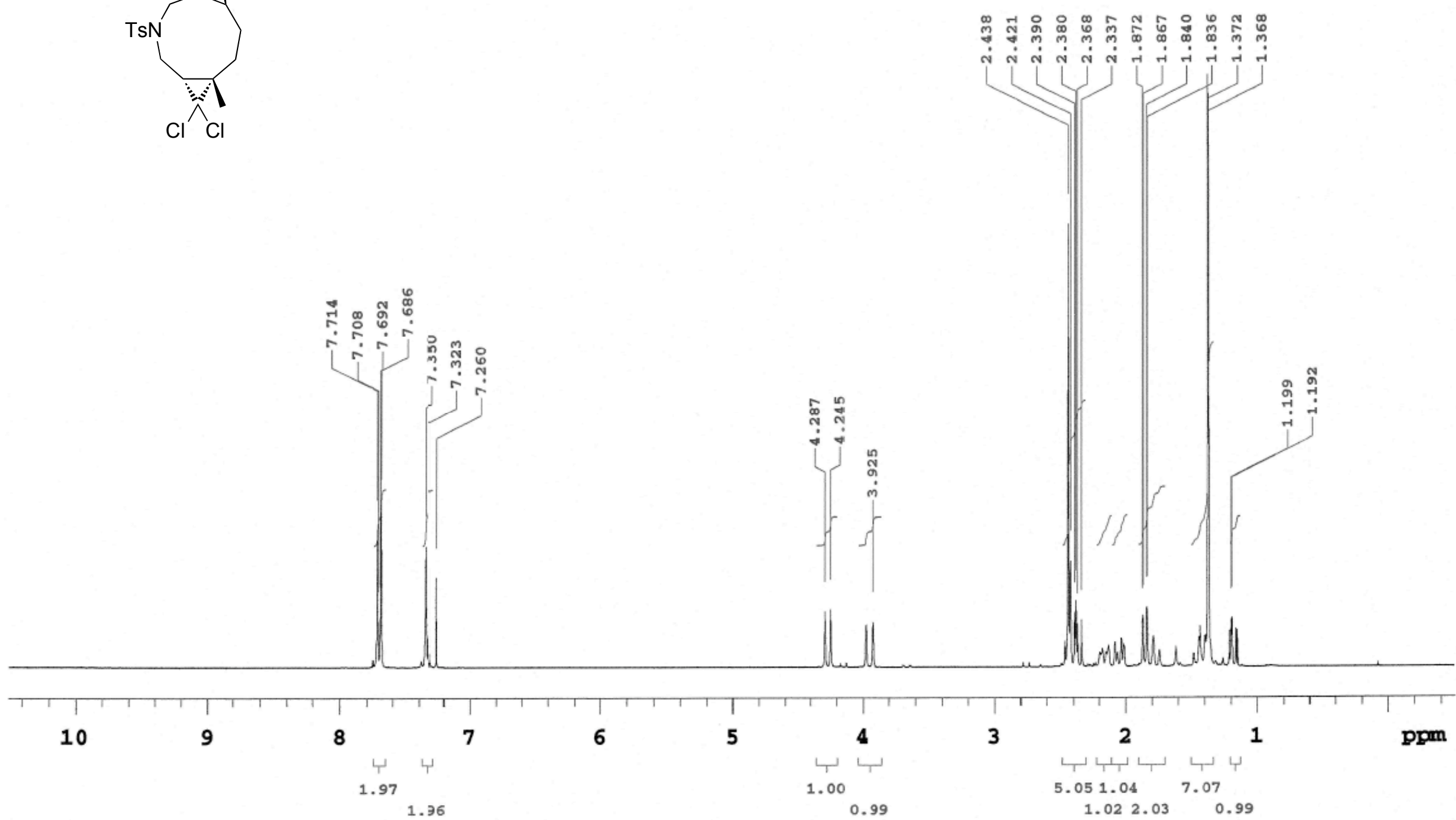
${ }^{13}$ C NMR Chart of $7 \mathbf{b}$
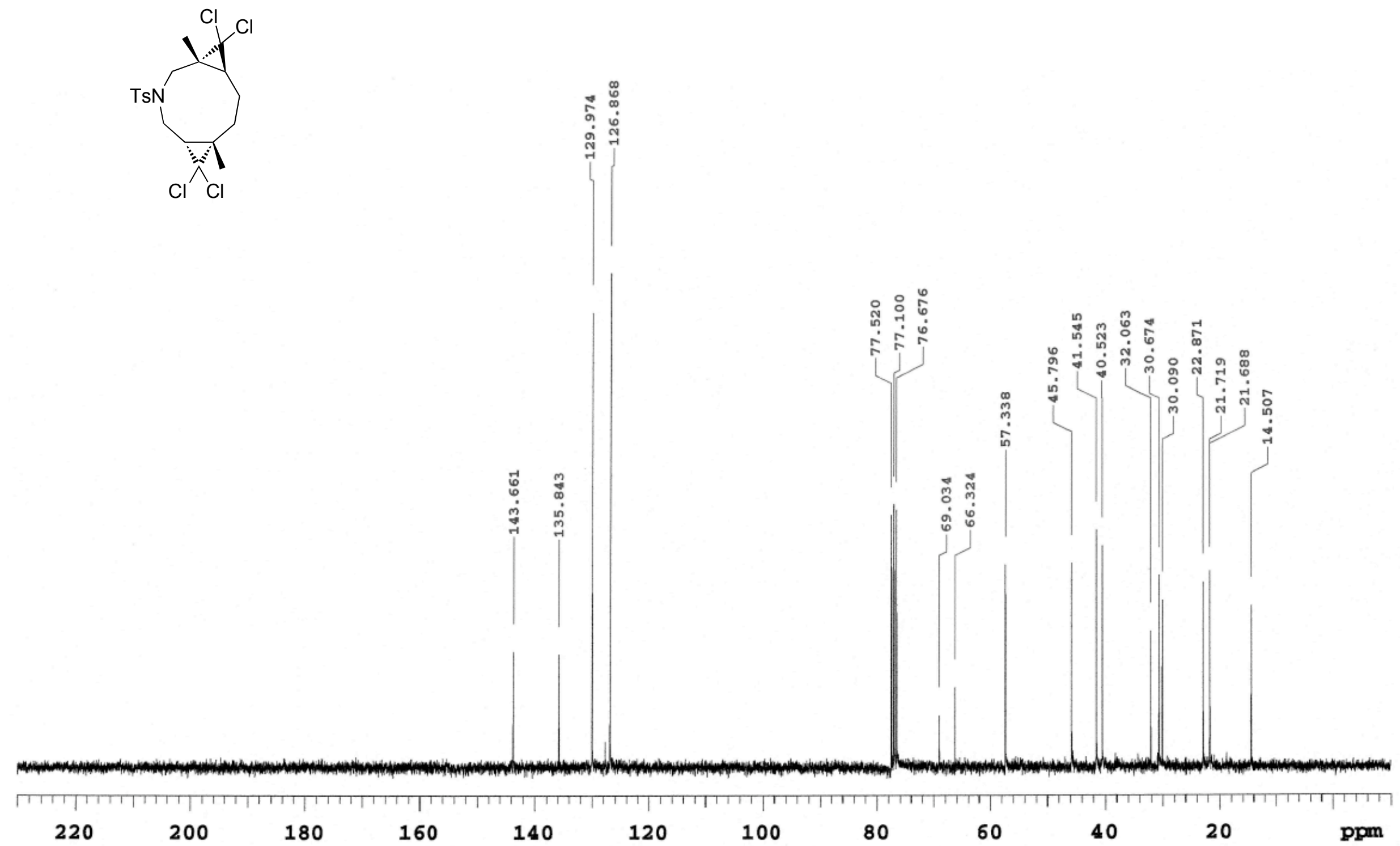


\section{${ }^{1}$ H NMR Chart of 8}

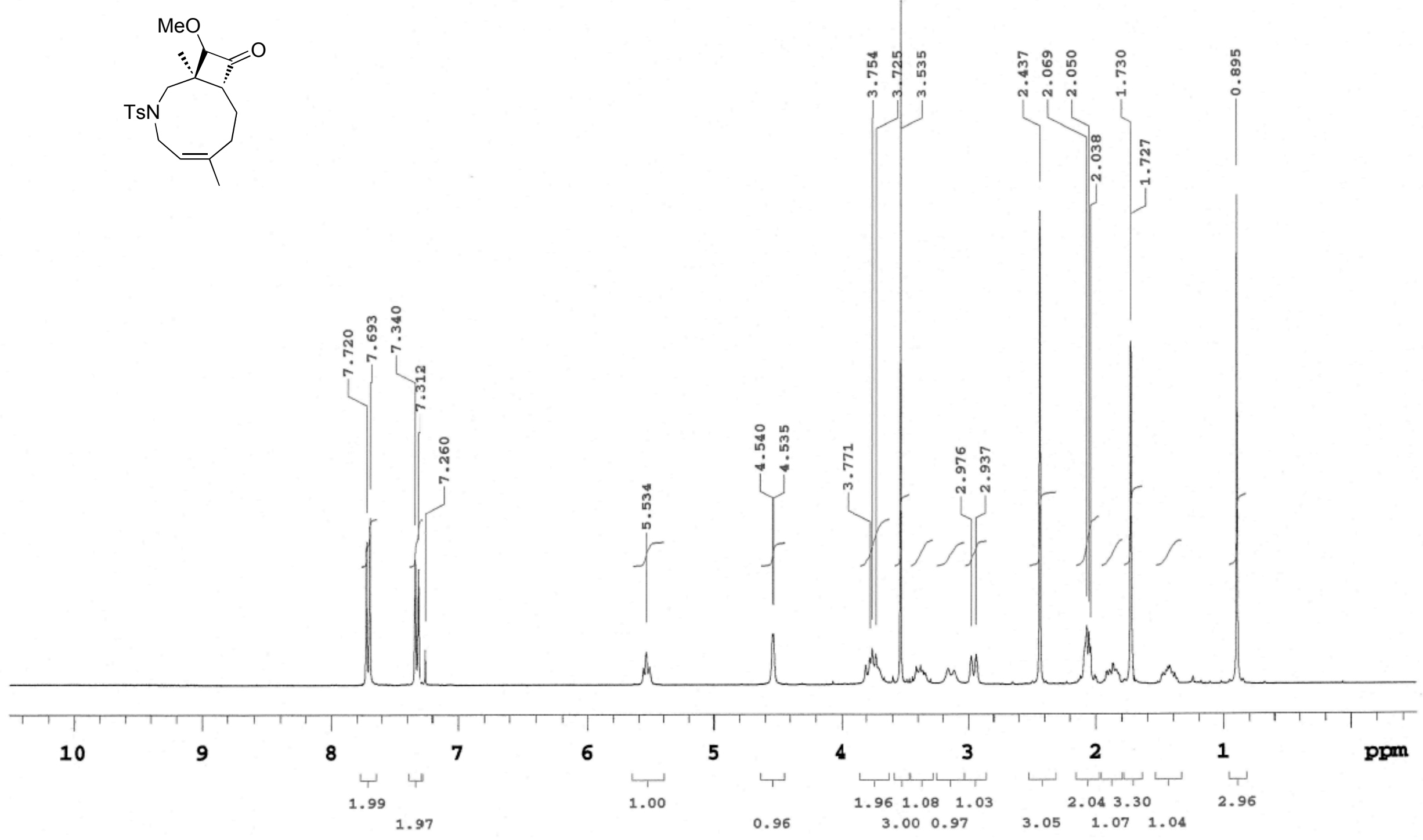


${ }^{13} \mathrm{C}$ NMR Chart of 8

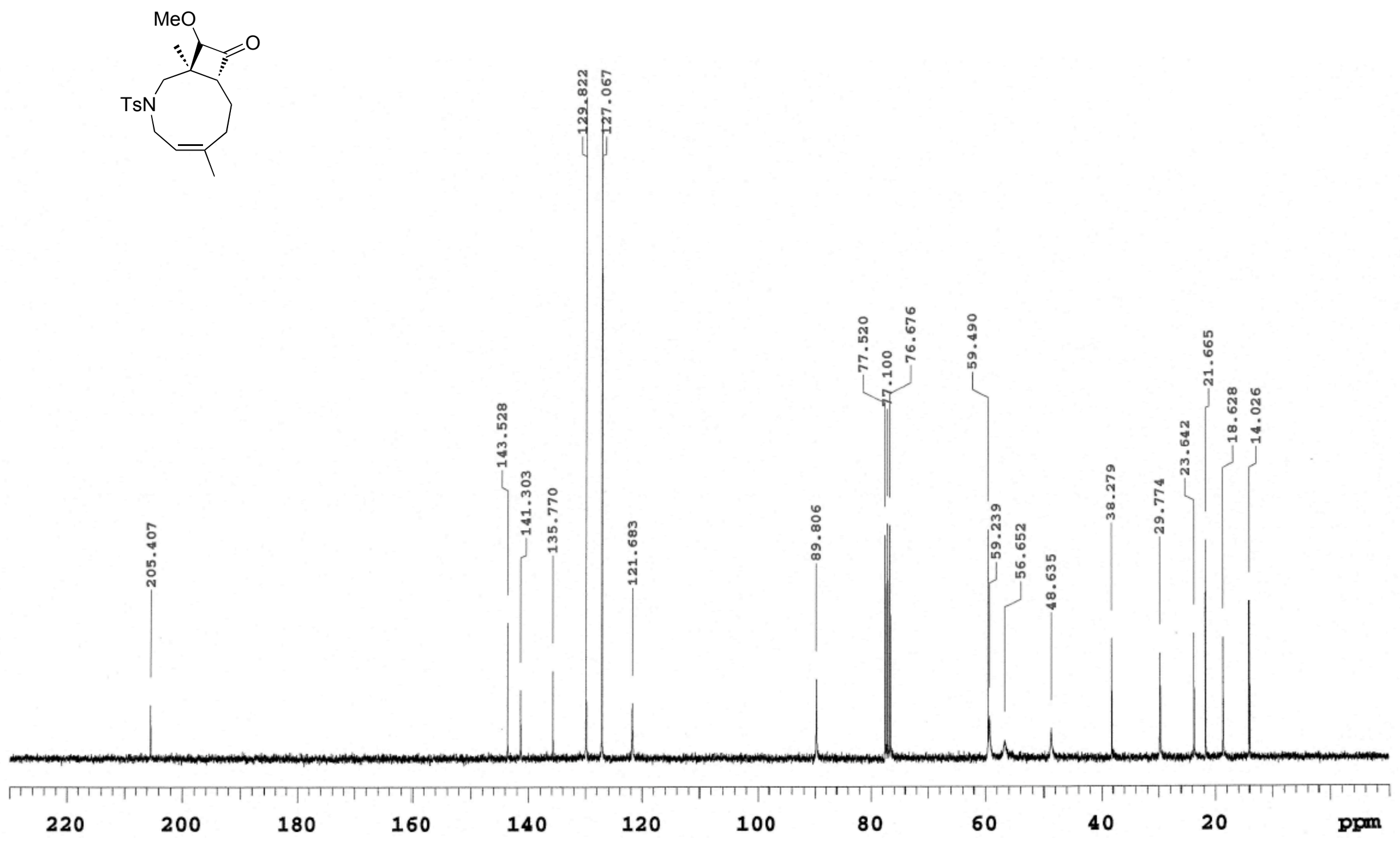


${ }^{1}$ H NMR Chart of 9a

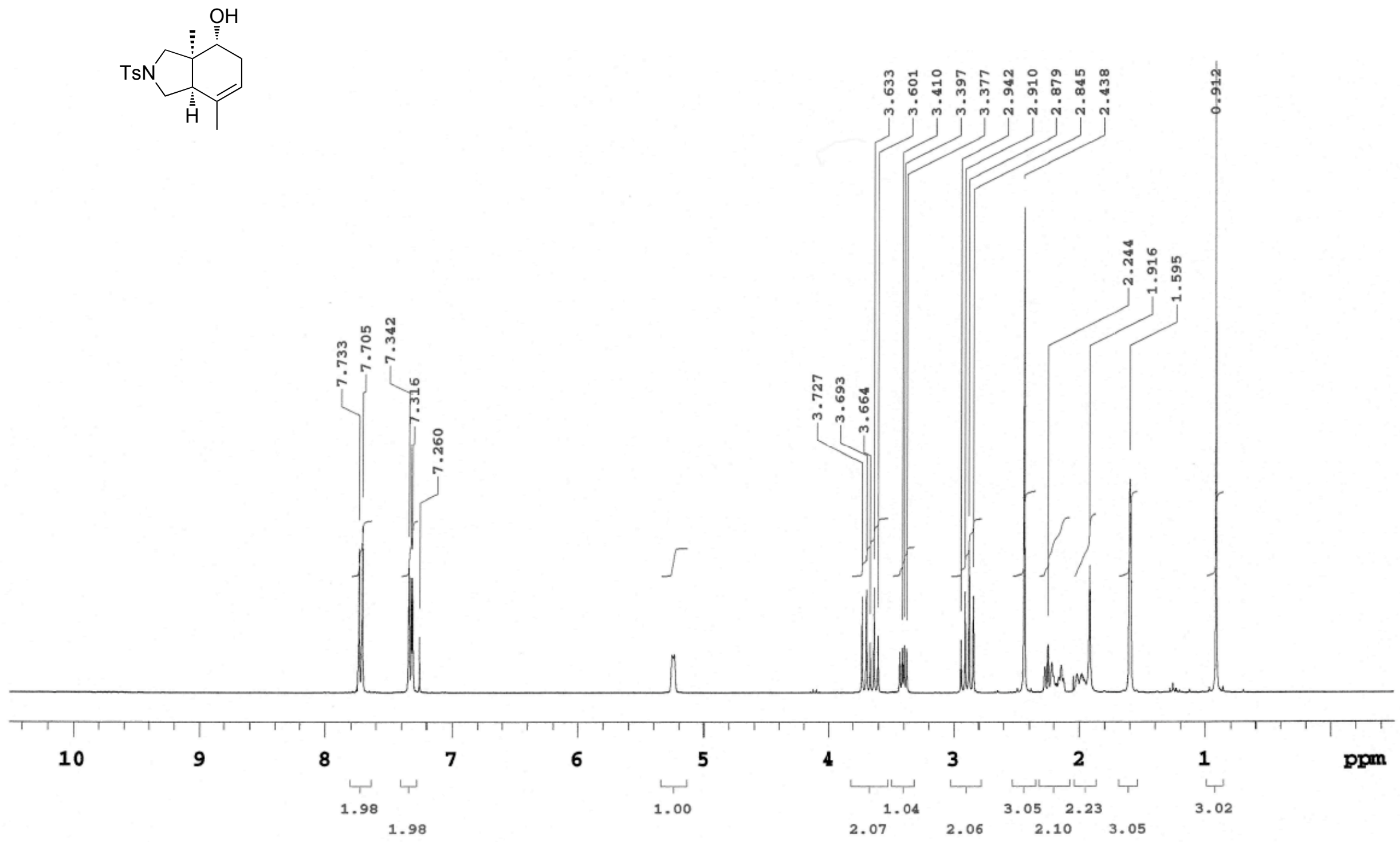


${ }^{13}$ C NMR Chart of 9a

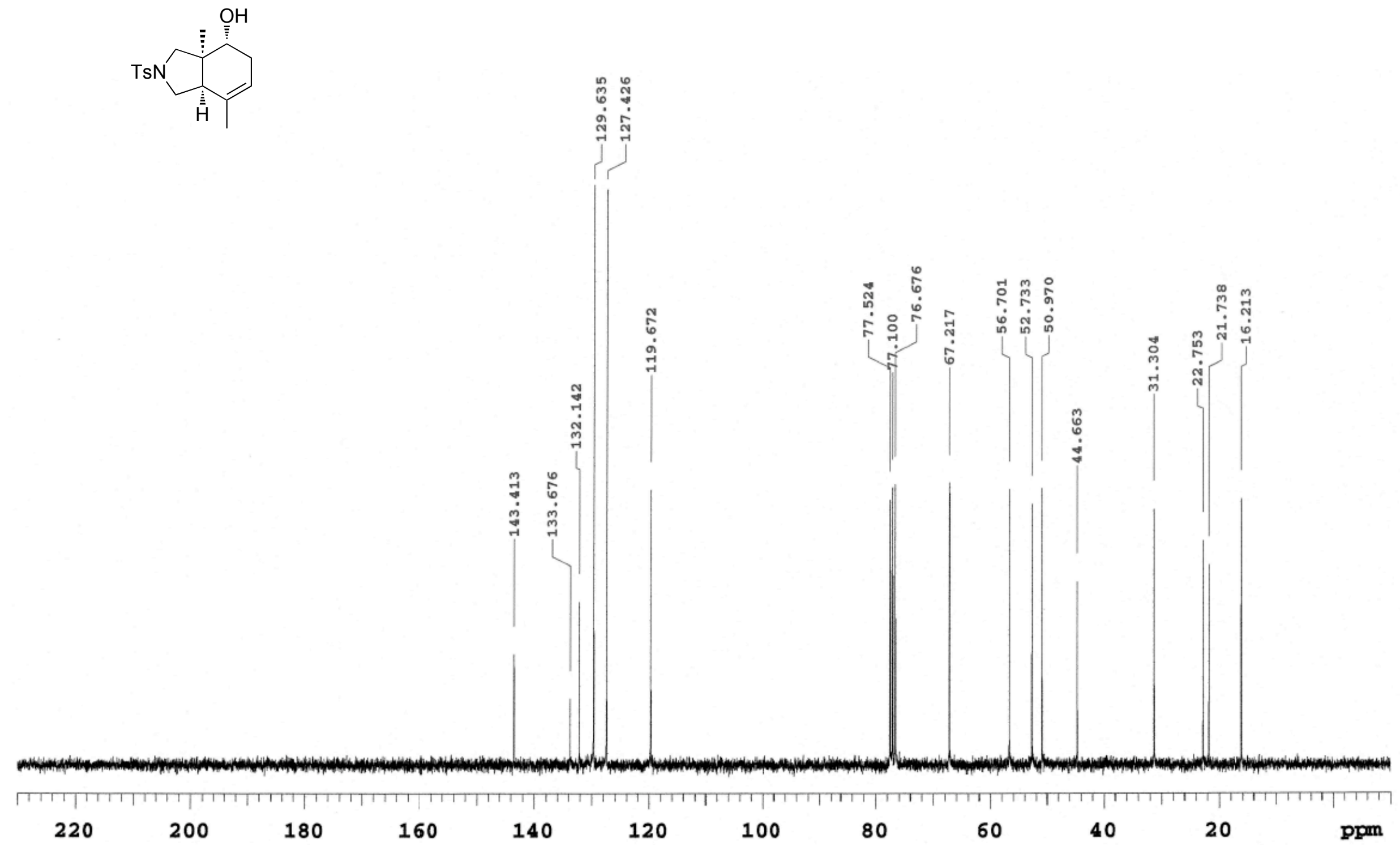


${ }^{1}$ H NMR Chart of 9b
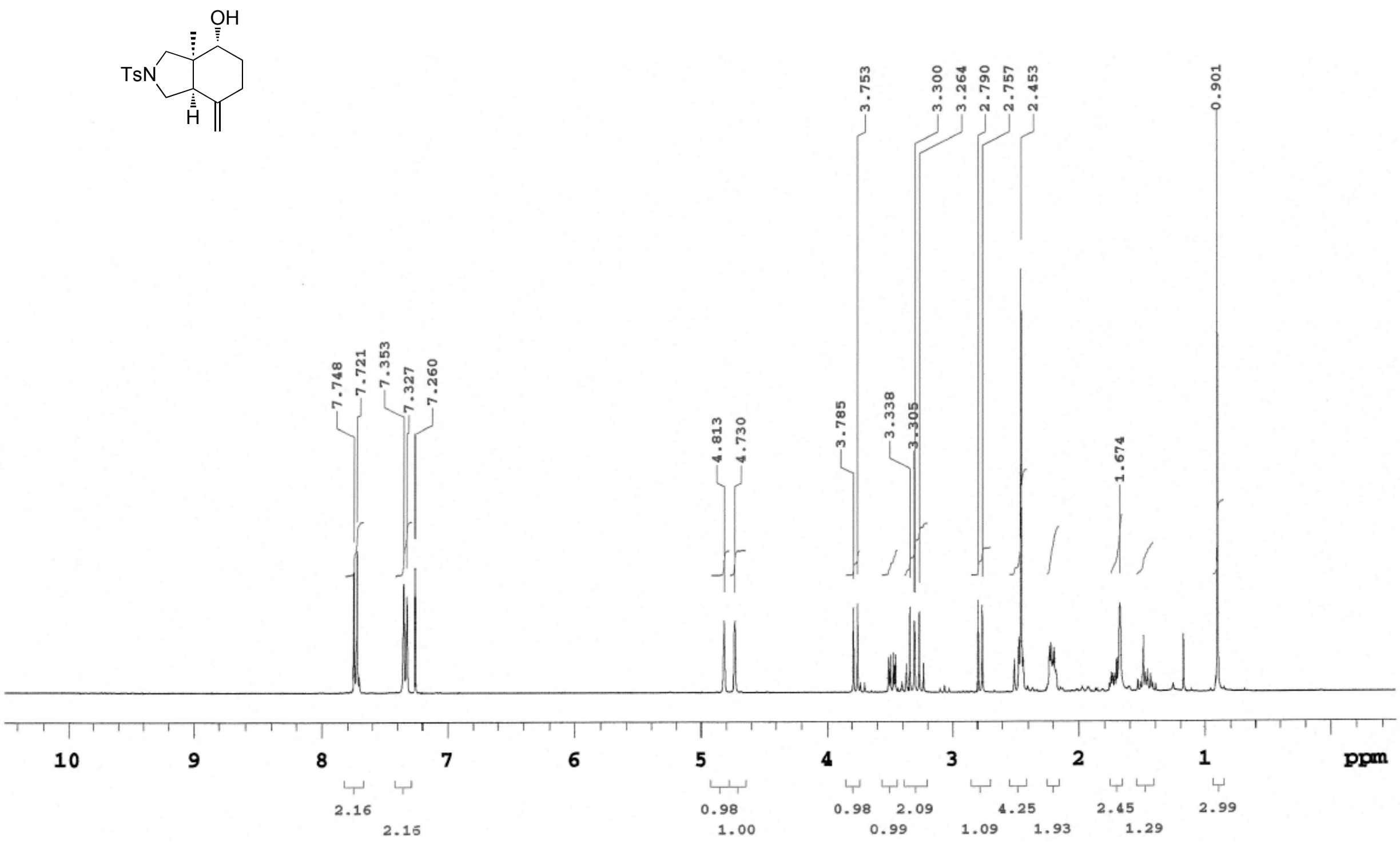
${ }^{13}$ C NMR Chart of 9b

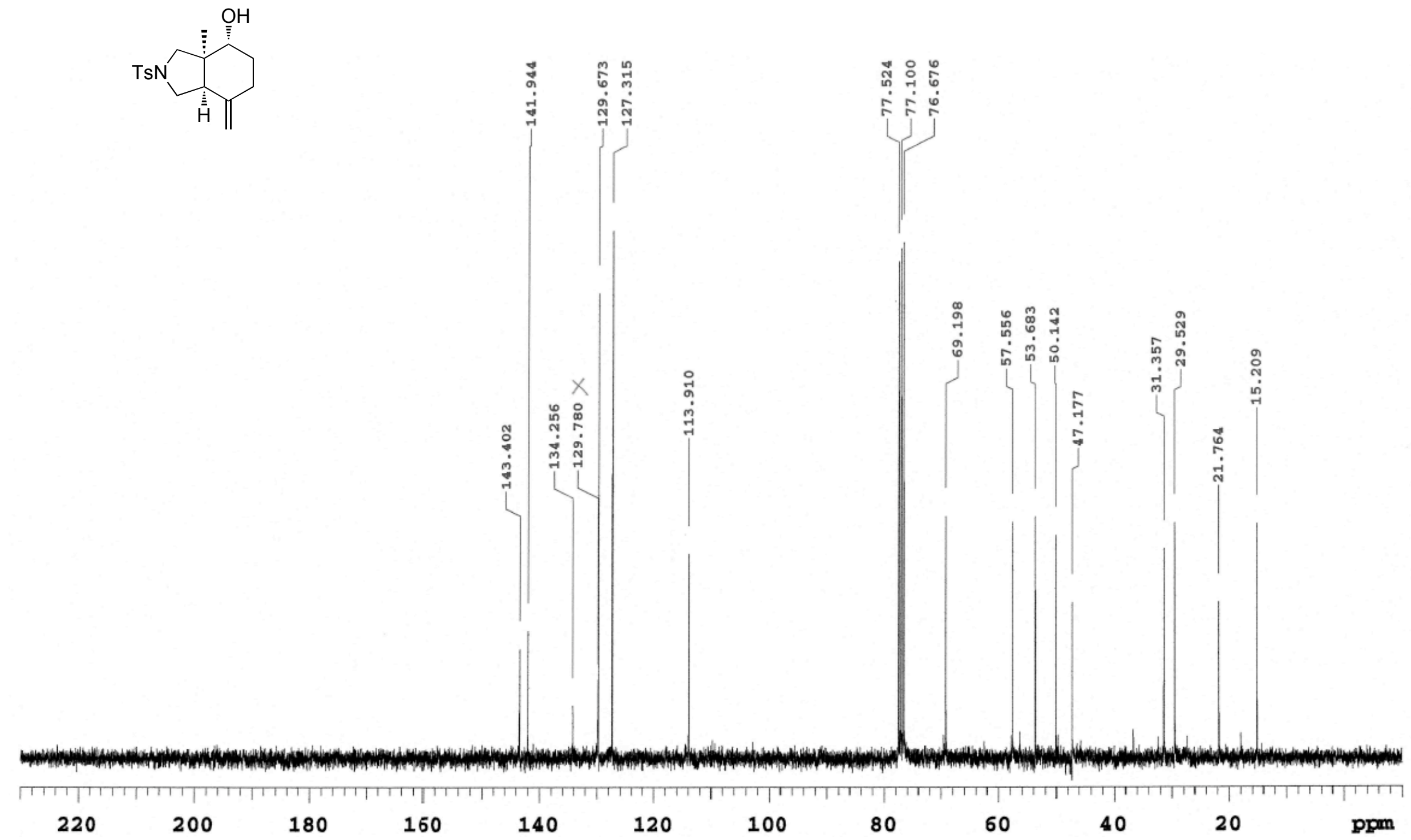


${ }^{1}$ H NMR Chart of 9c

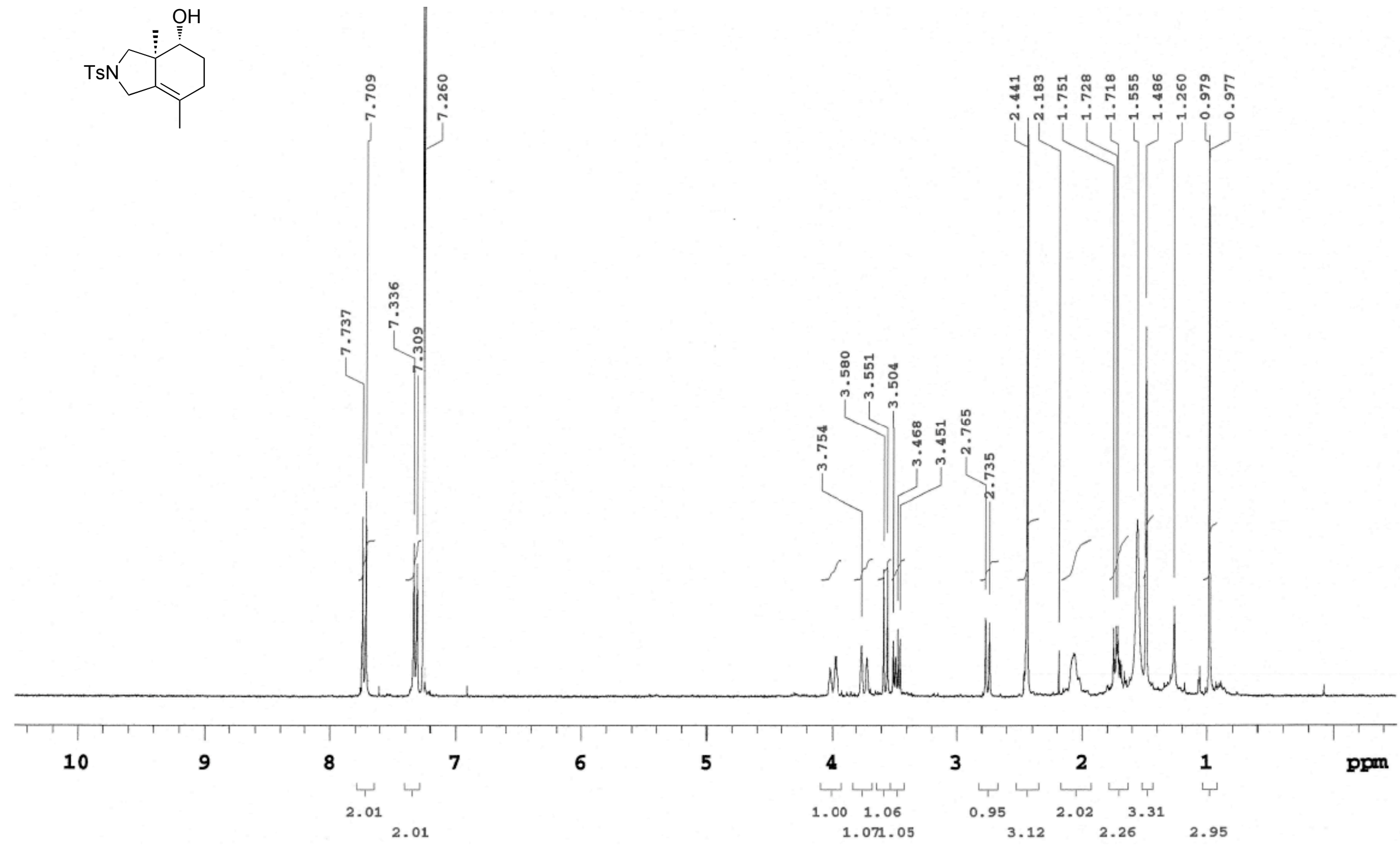


${ }^{13}$ C NMR Chart of 9c

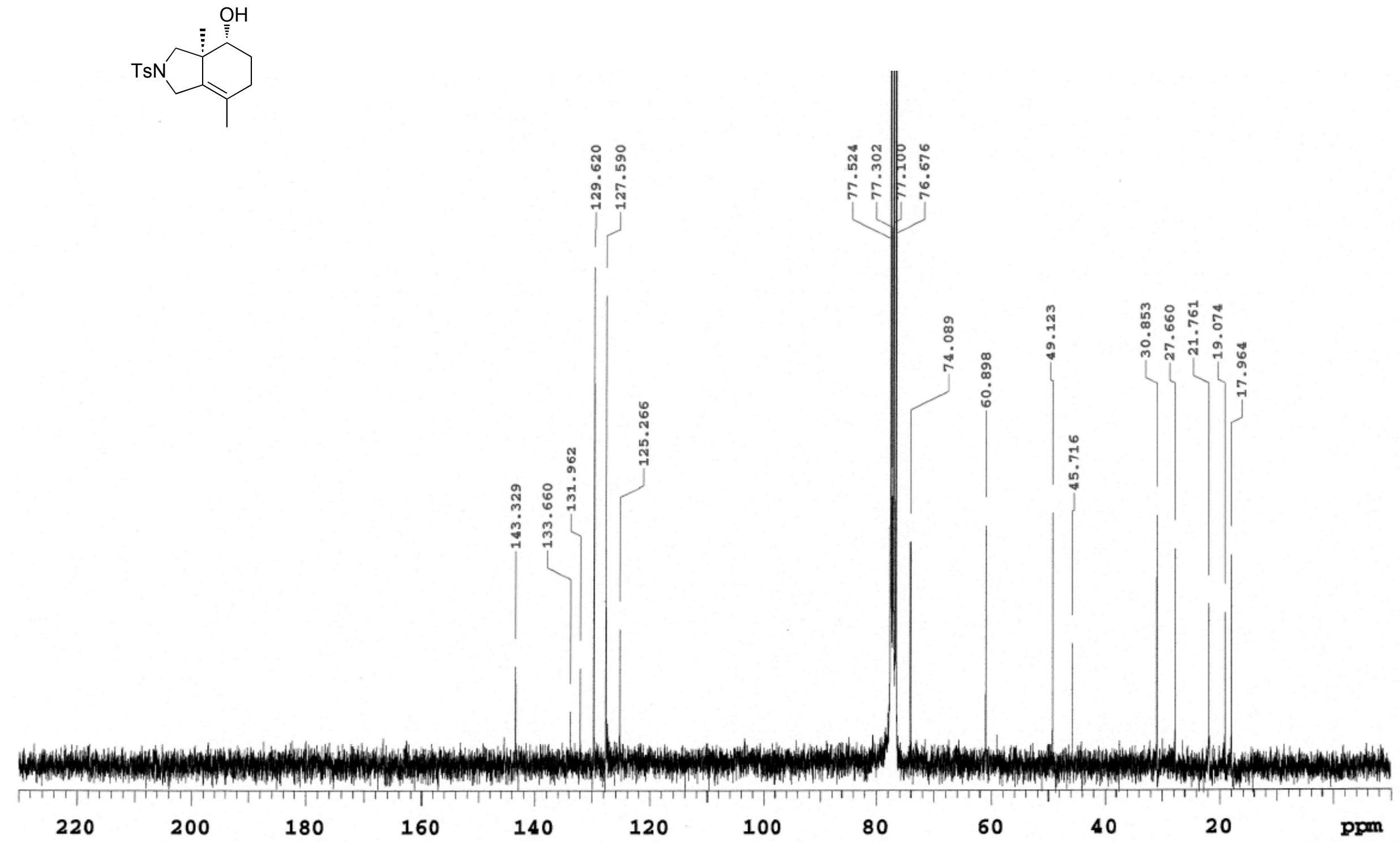




\section{${ }^{1} \mathrm{H}$ NMR Chart of 10a}

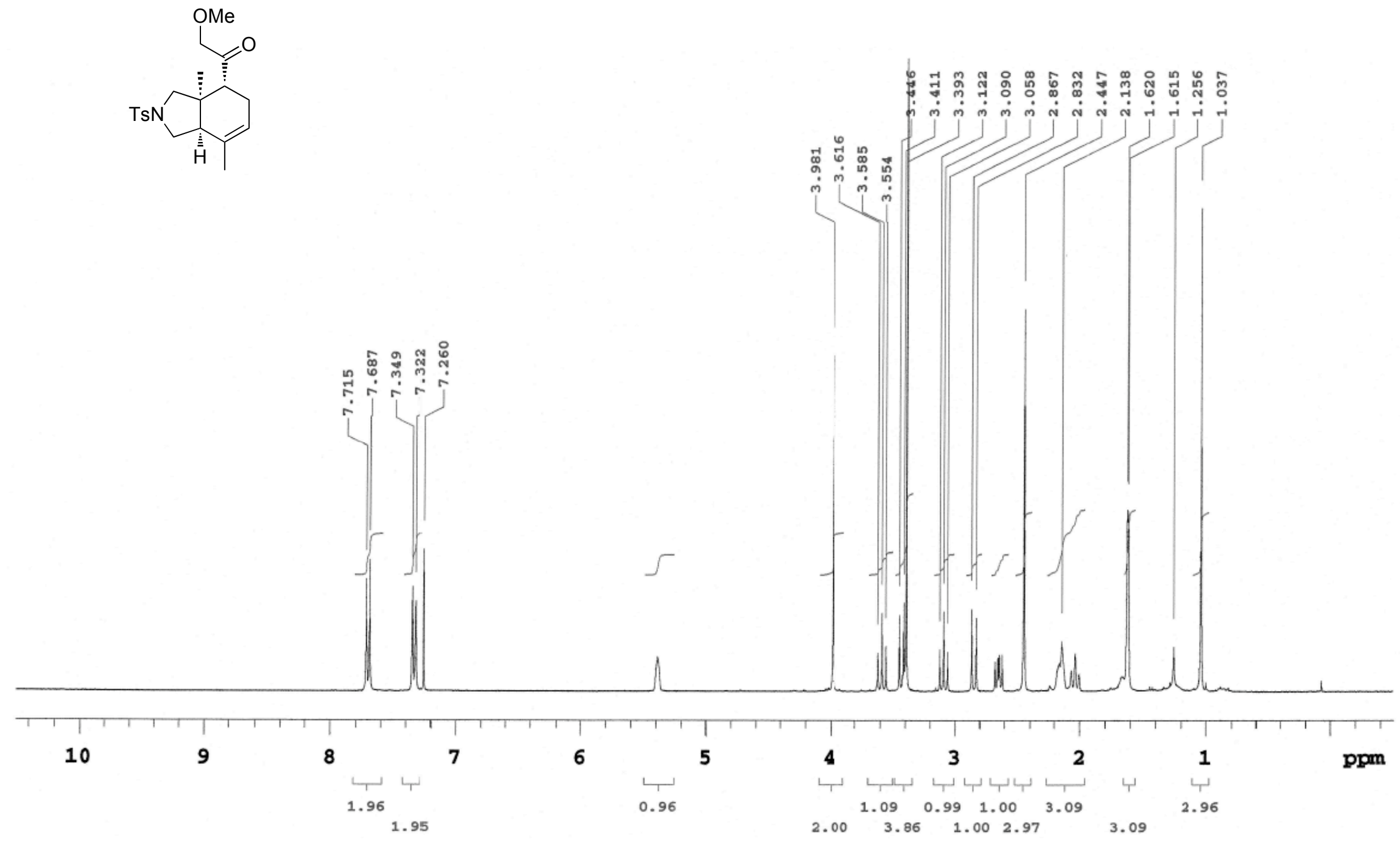


${ }^{13} \mathrm{C}$ NMR Chart of 10a

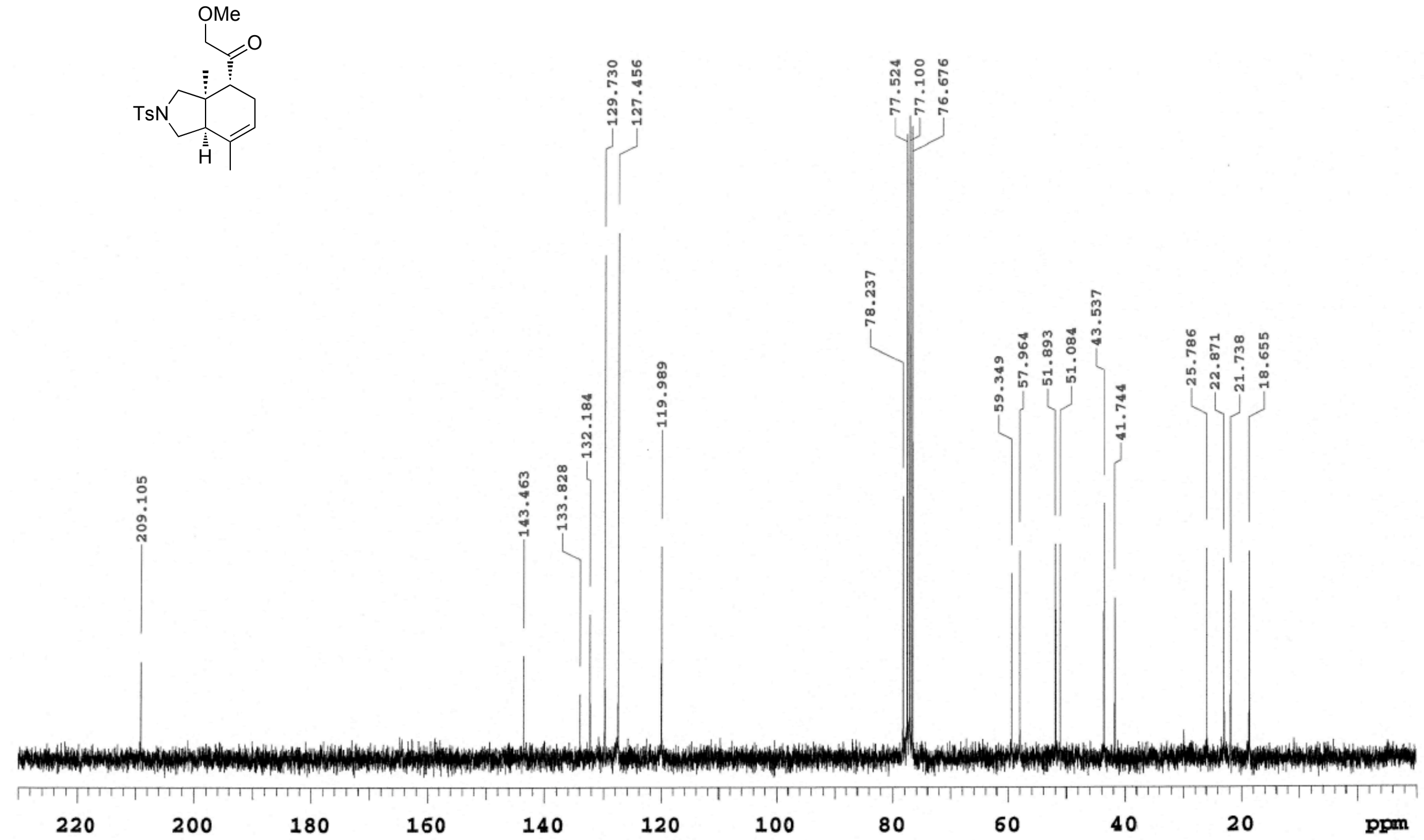




\section{${ }^{1} \mathrm{H}$ NMR Chart of 10c}

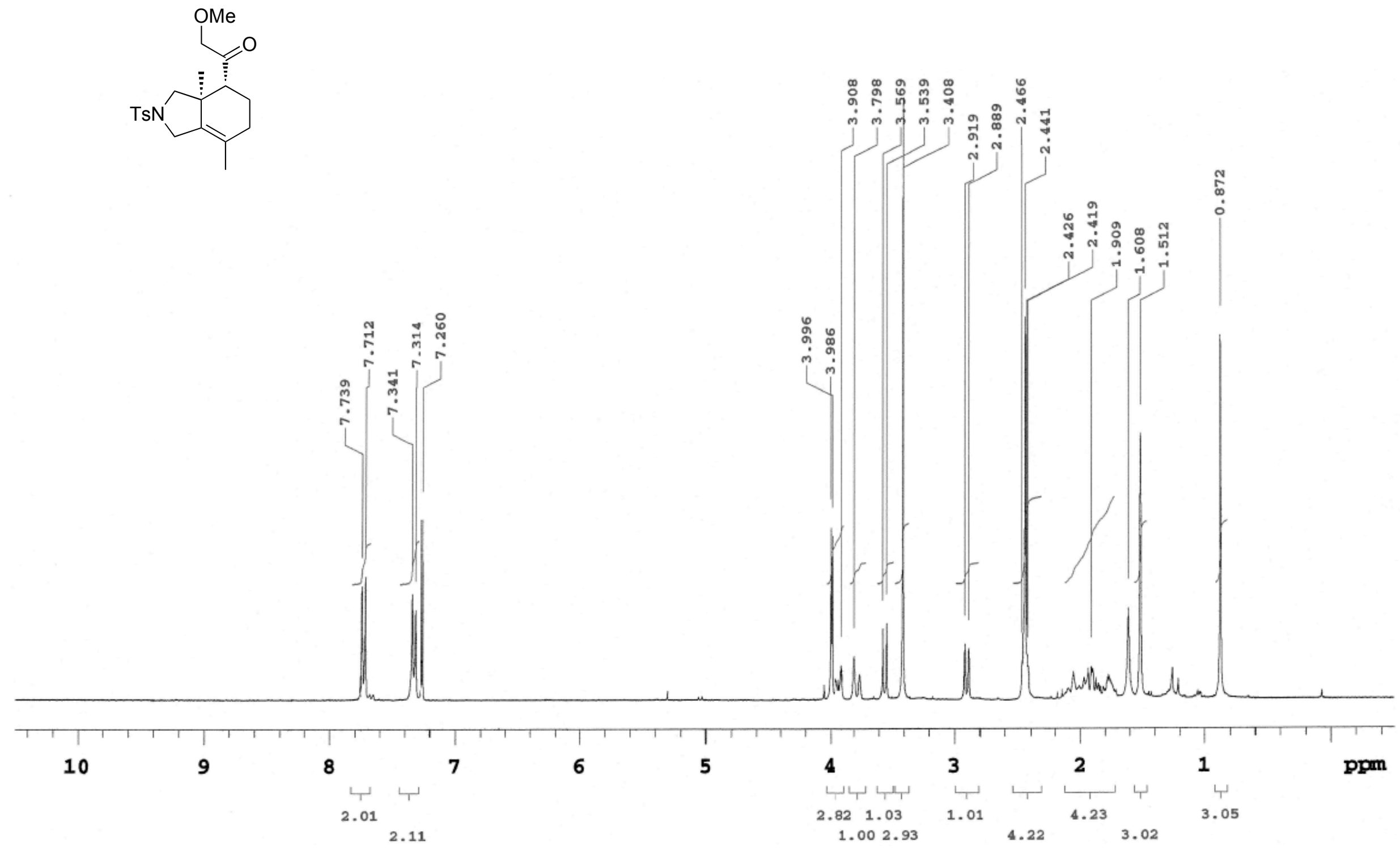


${ }^{13} \mathrm{C}$ NMR Chart of 10c

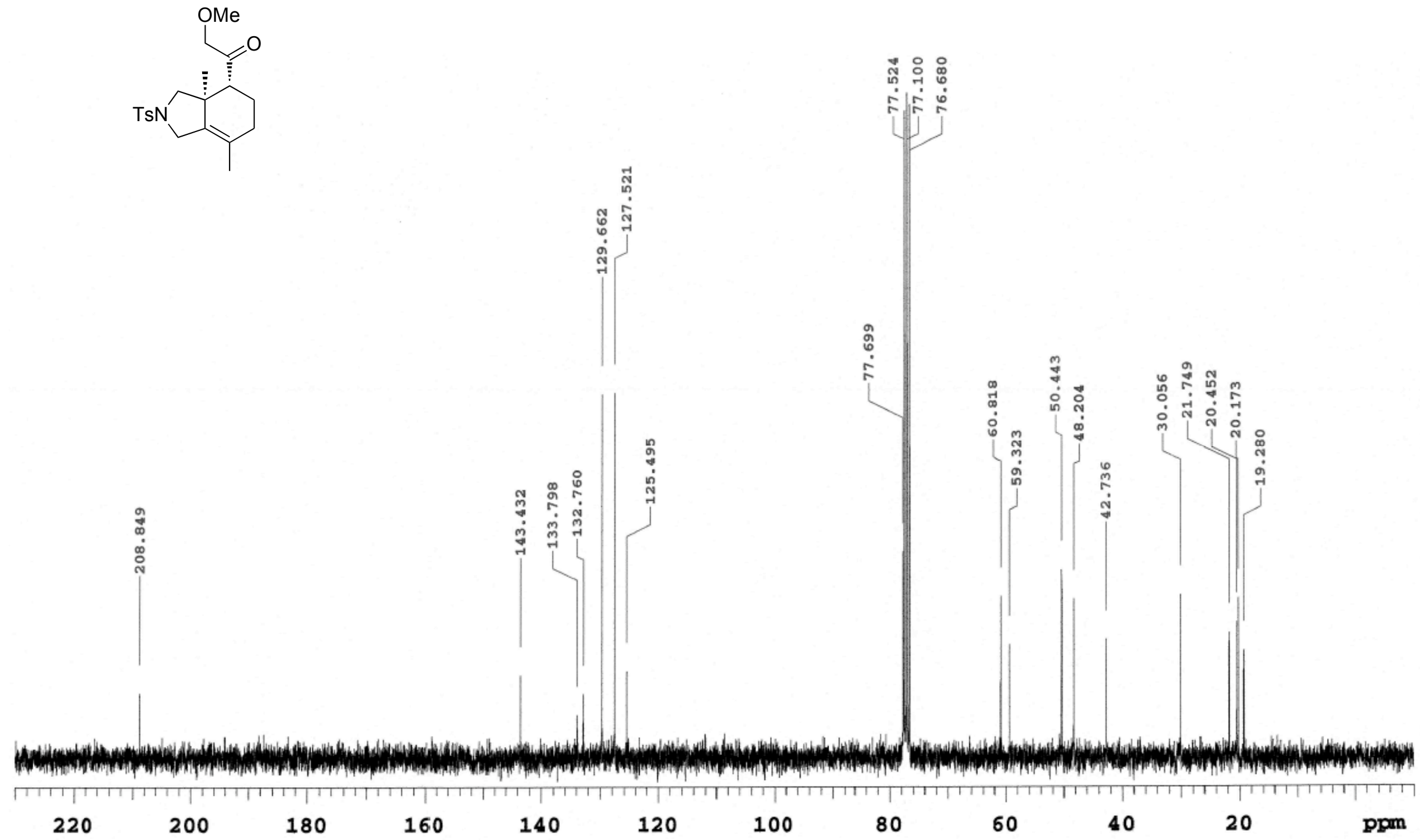


${ }^{1} \mathrm{H}$ NMR Chart of $\mathbf{1 1}$

承

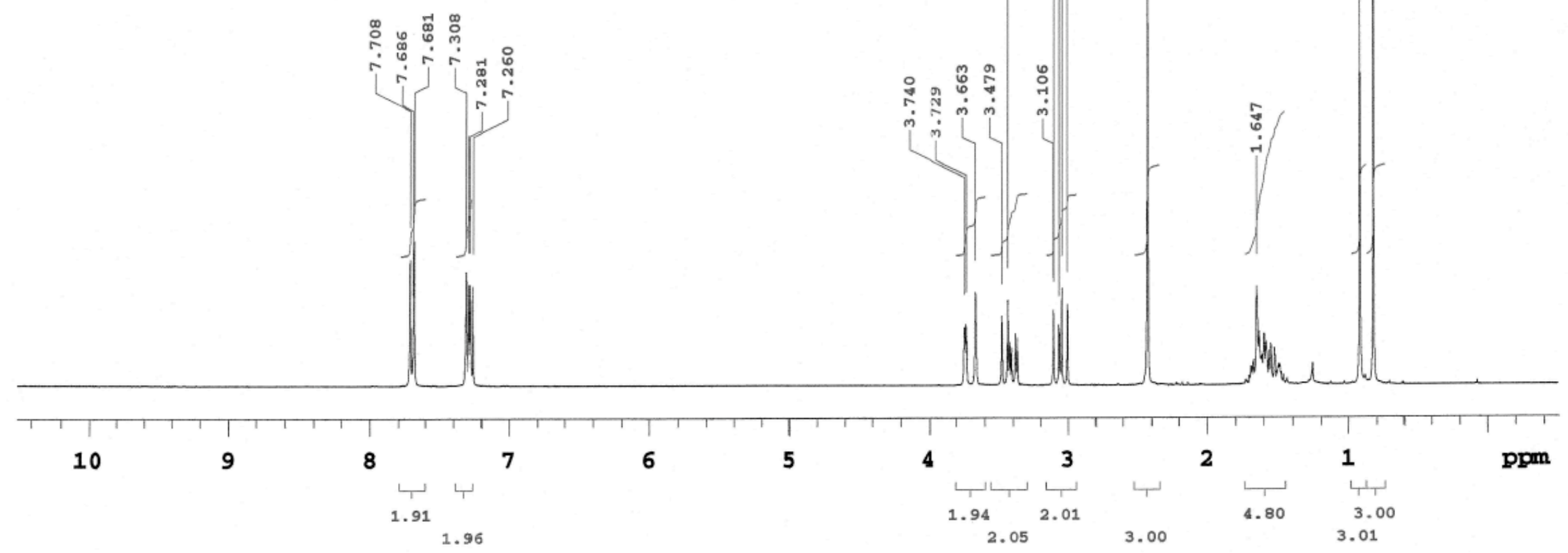


${ }^{13} \mathrm{C}$ NMR Chart of $\mathbf{1 1}$

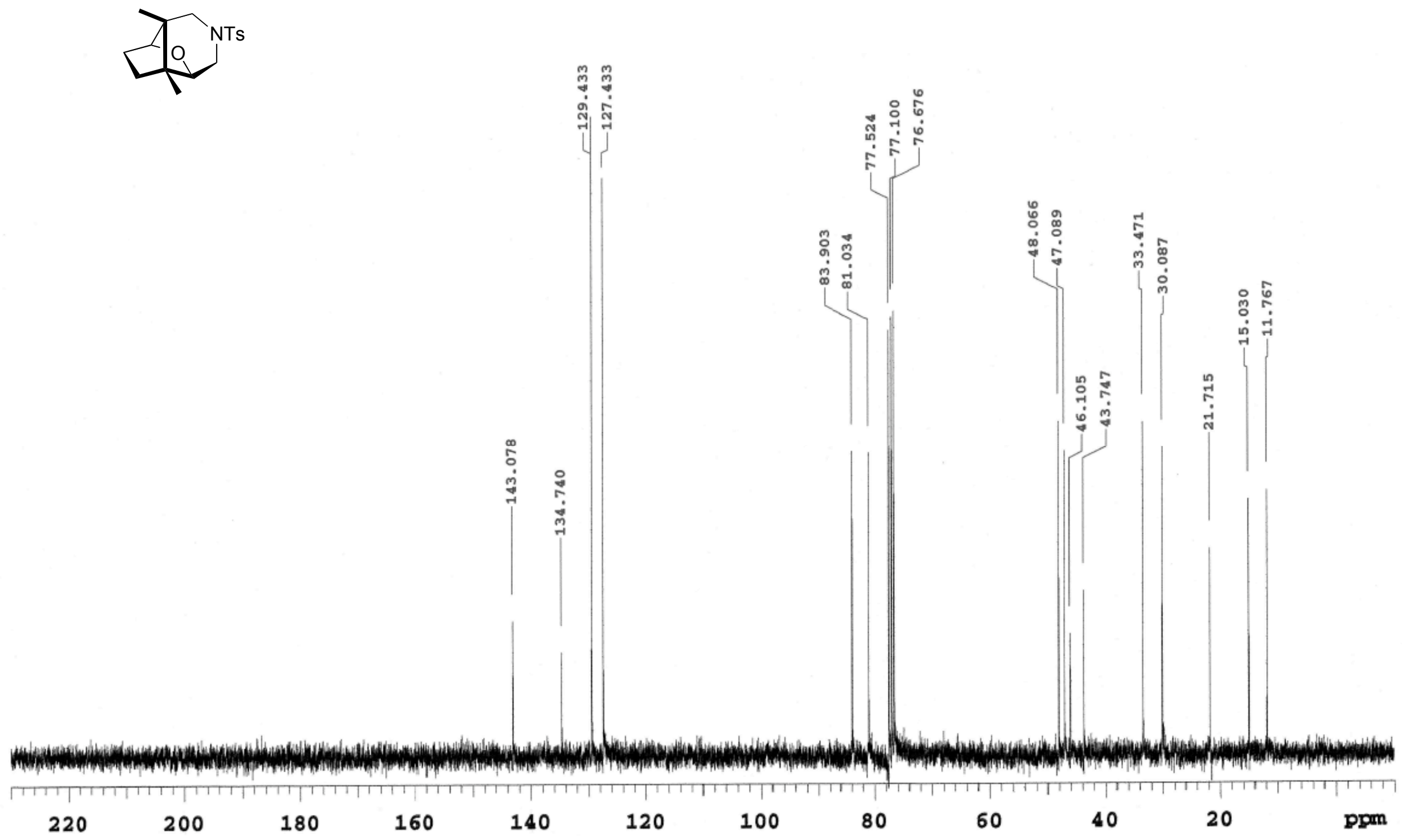


${ }^{1} \mathrm{H}$ NMR Chart of 12a

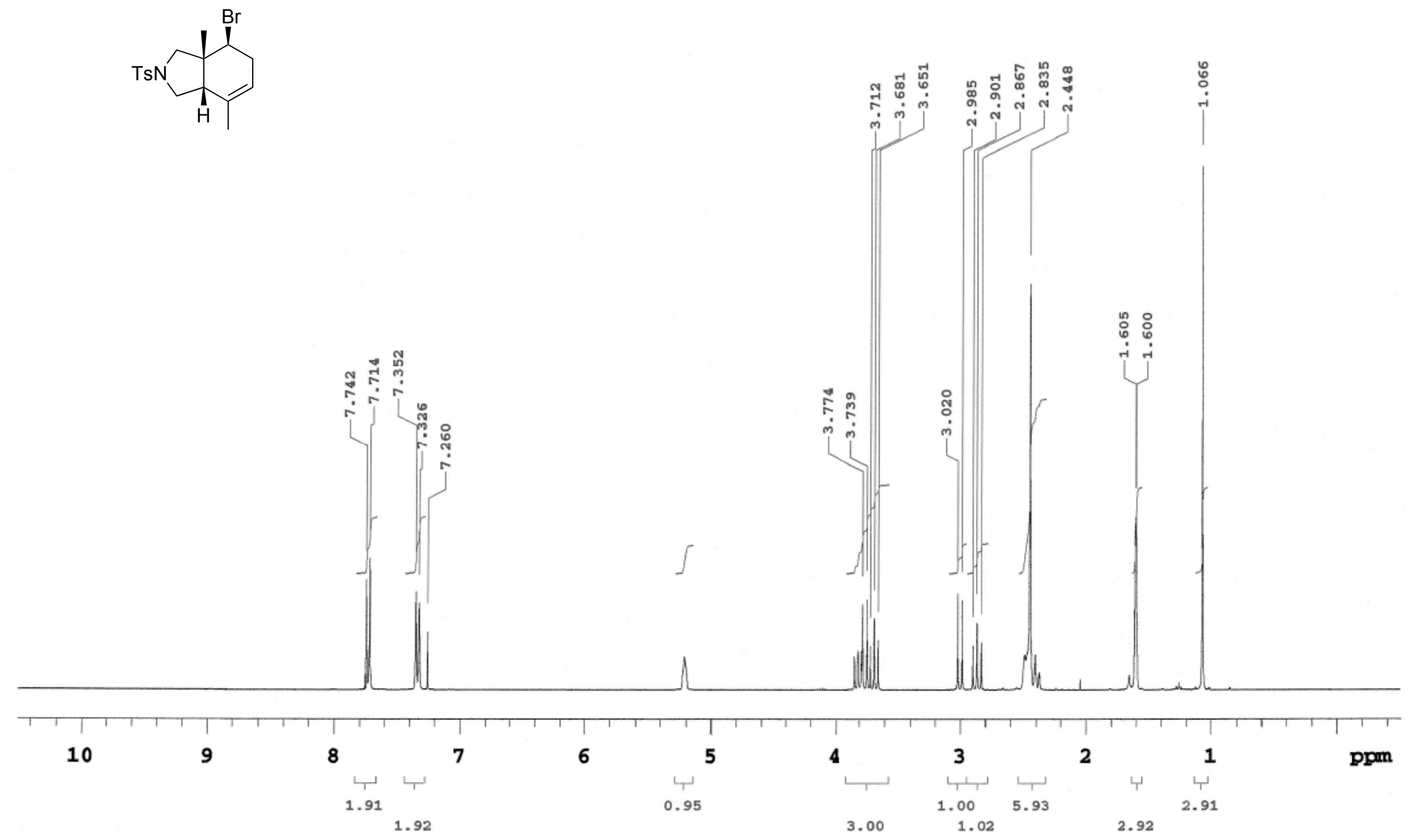


${ }^{13} \mathrm{C}$ NMR Chart of 12a

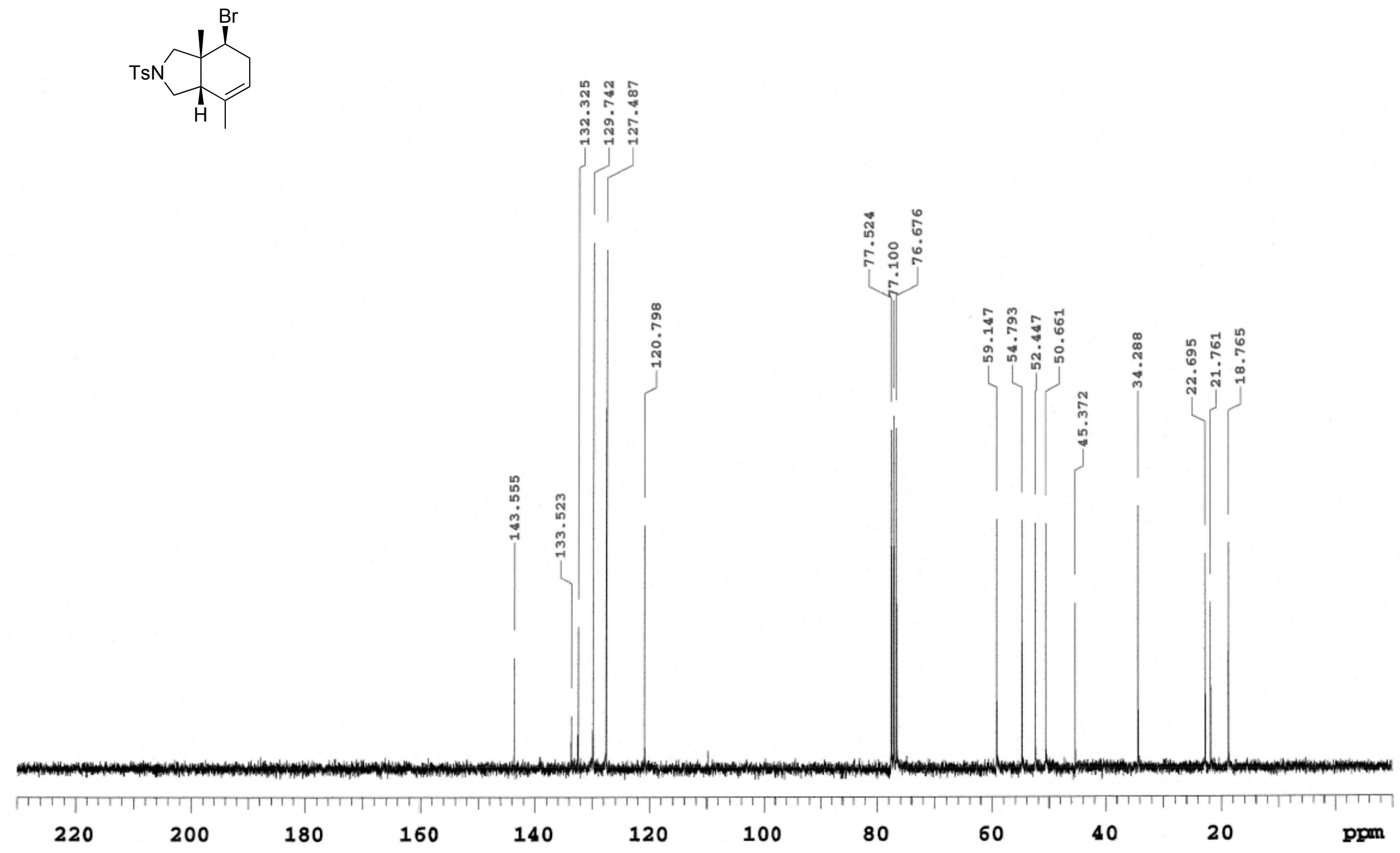


${ }^{1} \mathrm{H}$ NMR Chart of $\mathbf{1 2 b}$

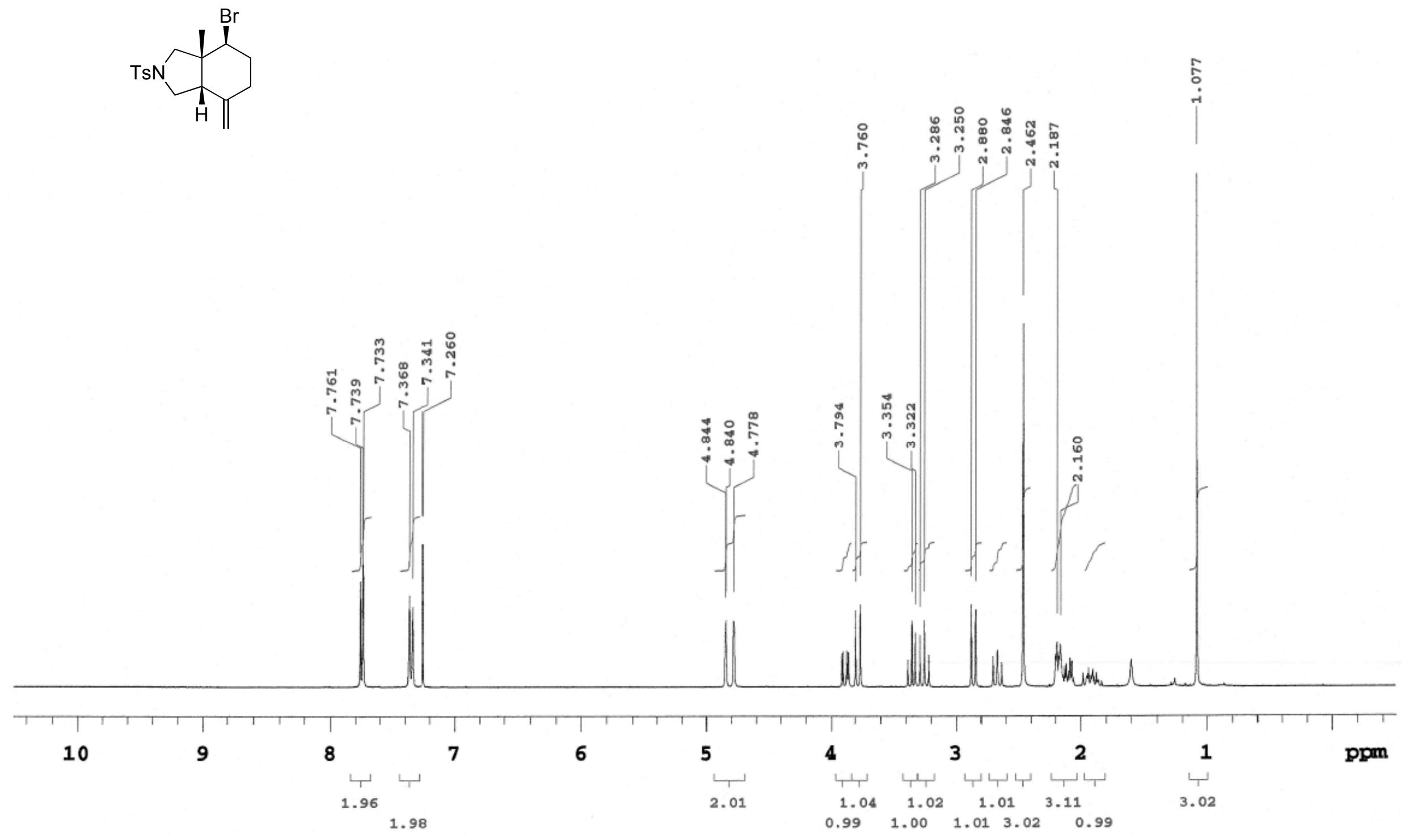


${ }^{13} \mathrm{C}$ NMR Chart of $\mathbf{1 2 b}$

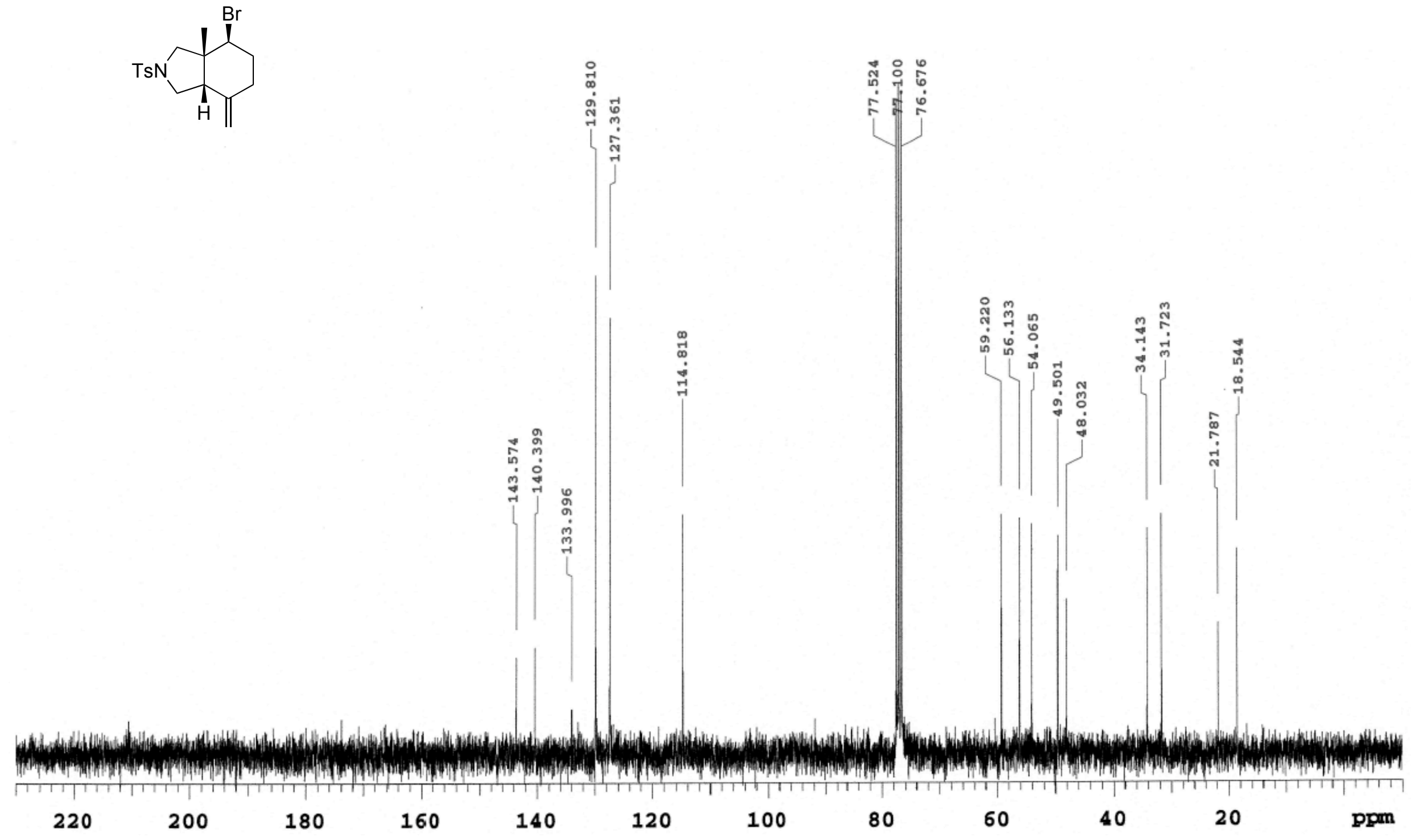


${ }^{1} \mathrm{H}$ NMR Chart of 12c
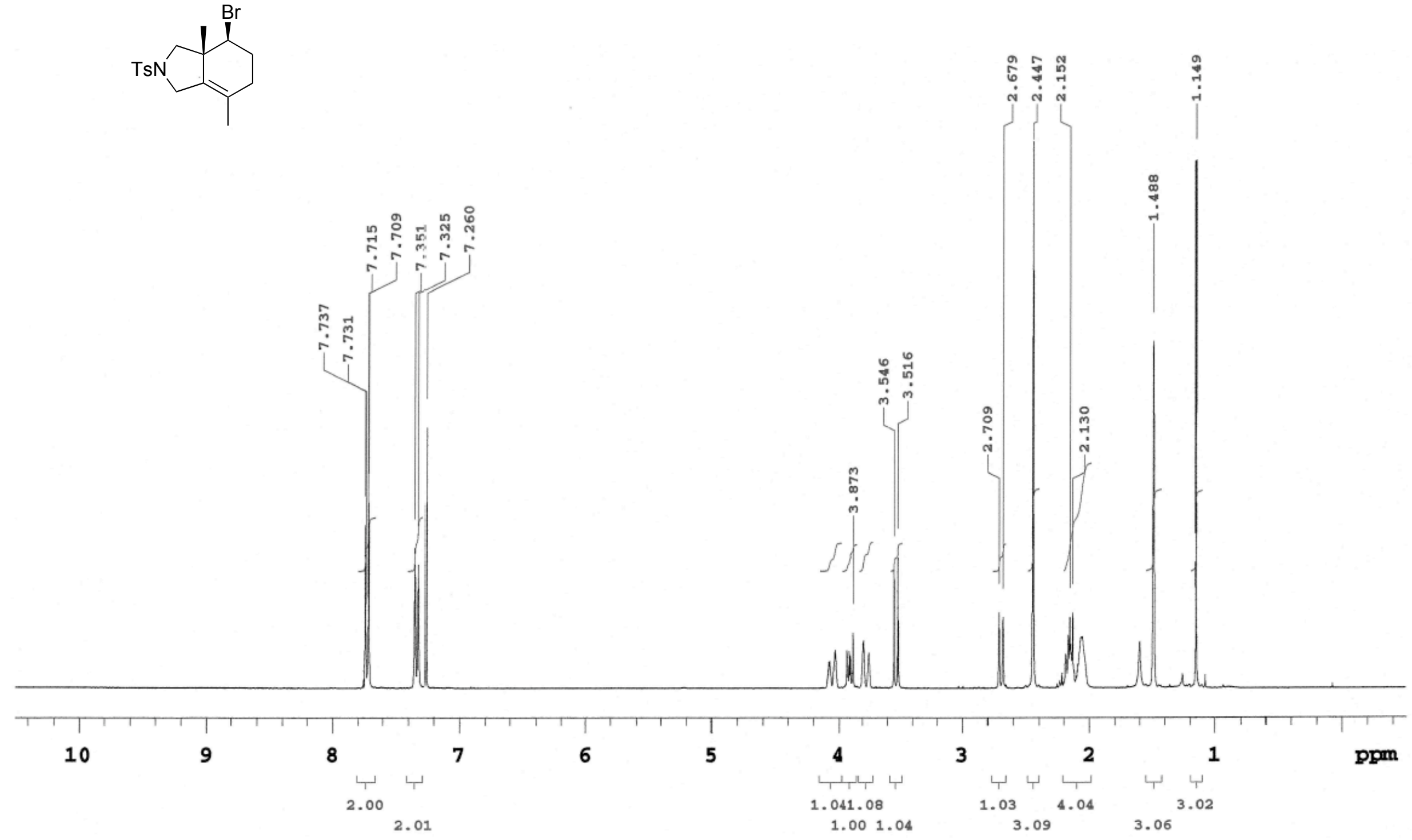
${ }^{13}$ C NMR Chart of 12c

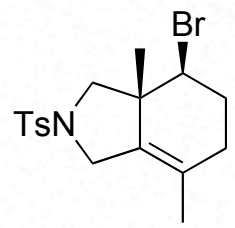

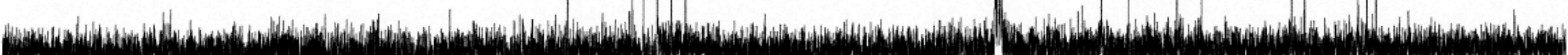

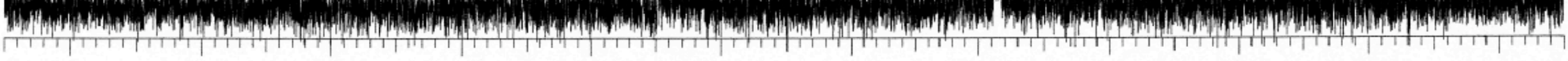




\section{${ }^{1} \mathrm{H}$ NMR Chart of 13a}

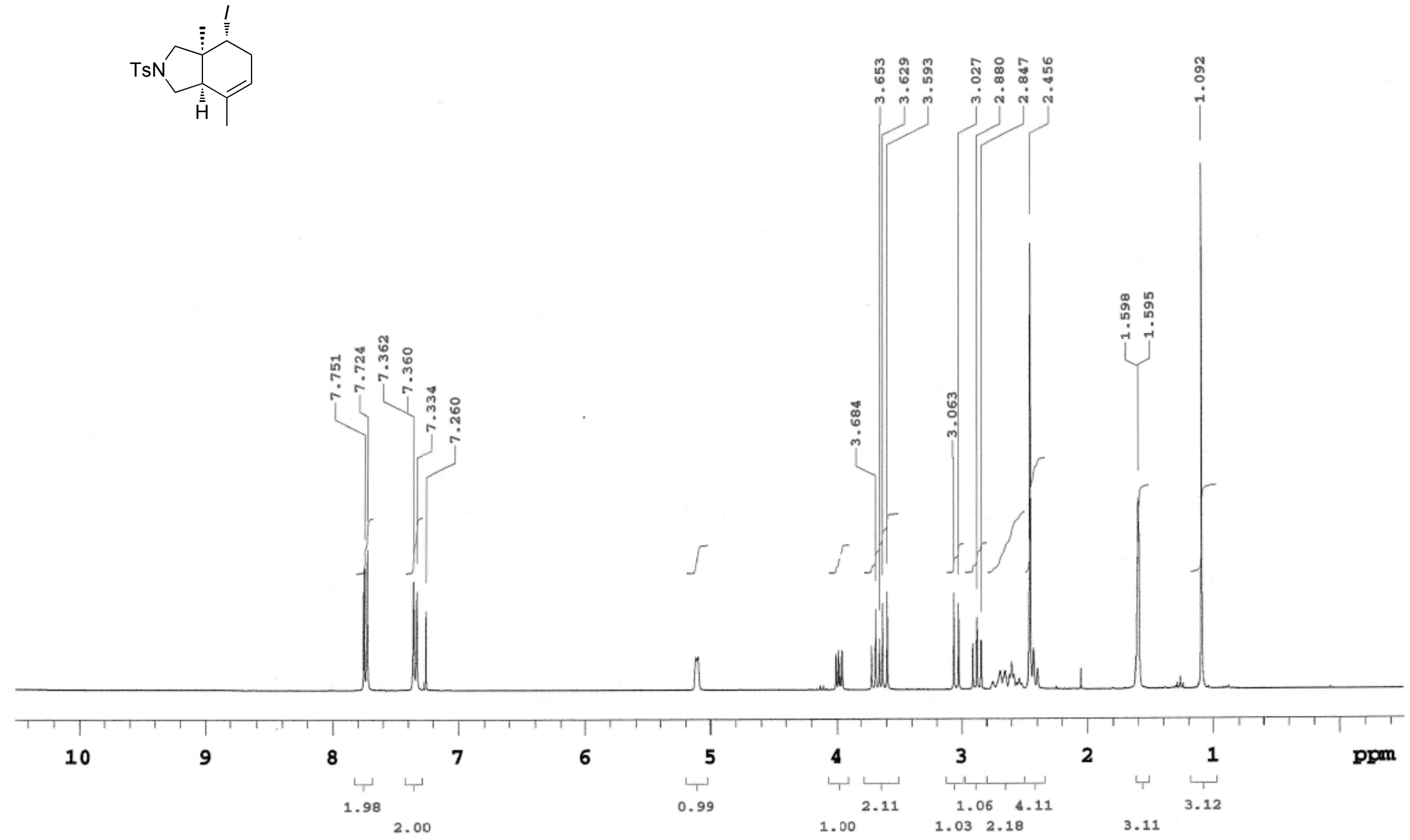


${ }^{13} \mathrm{C}$ NMR Chart of 13a
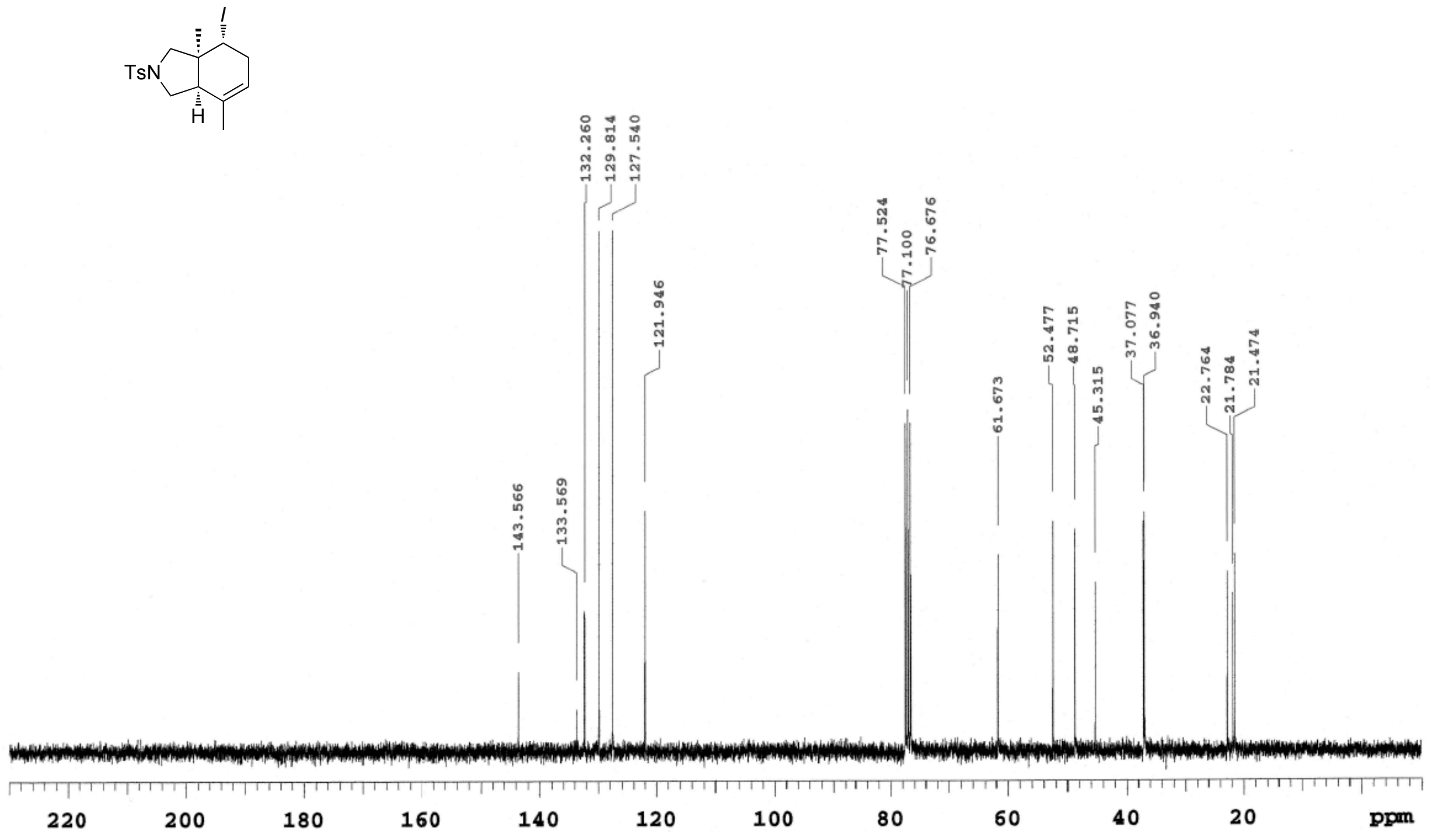
${ }^{1} \mathrm{H}$ NMR Chart of $\mathbf{1 3 b}$
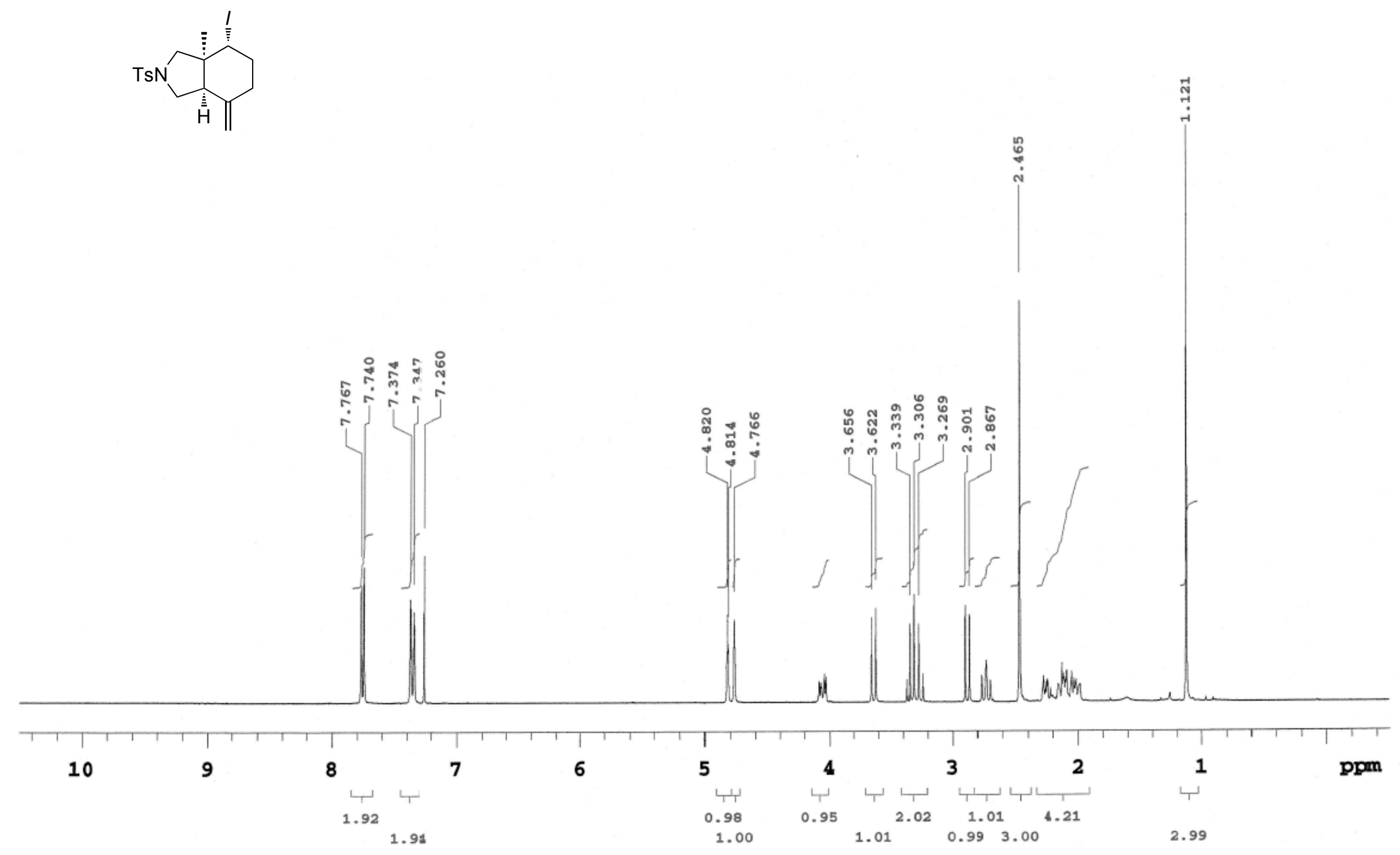
${ }^{13} \mathrm{C}$ NMR Chart of $\mathbf{1 3 b}$

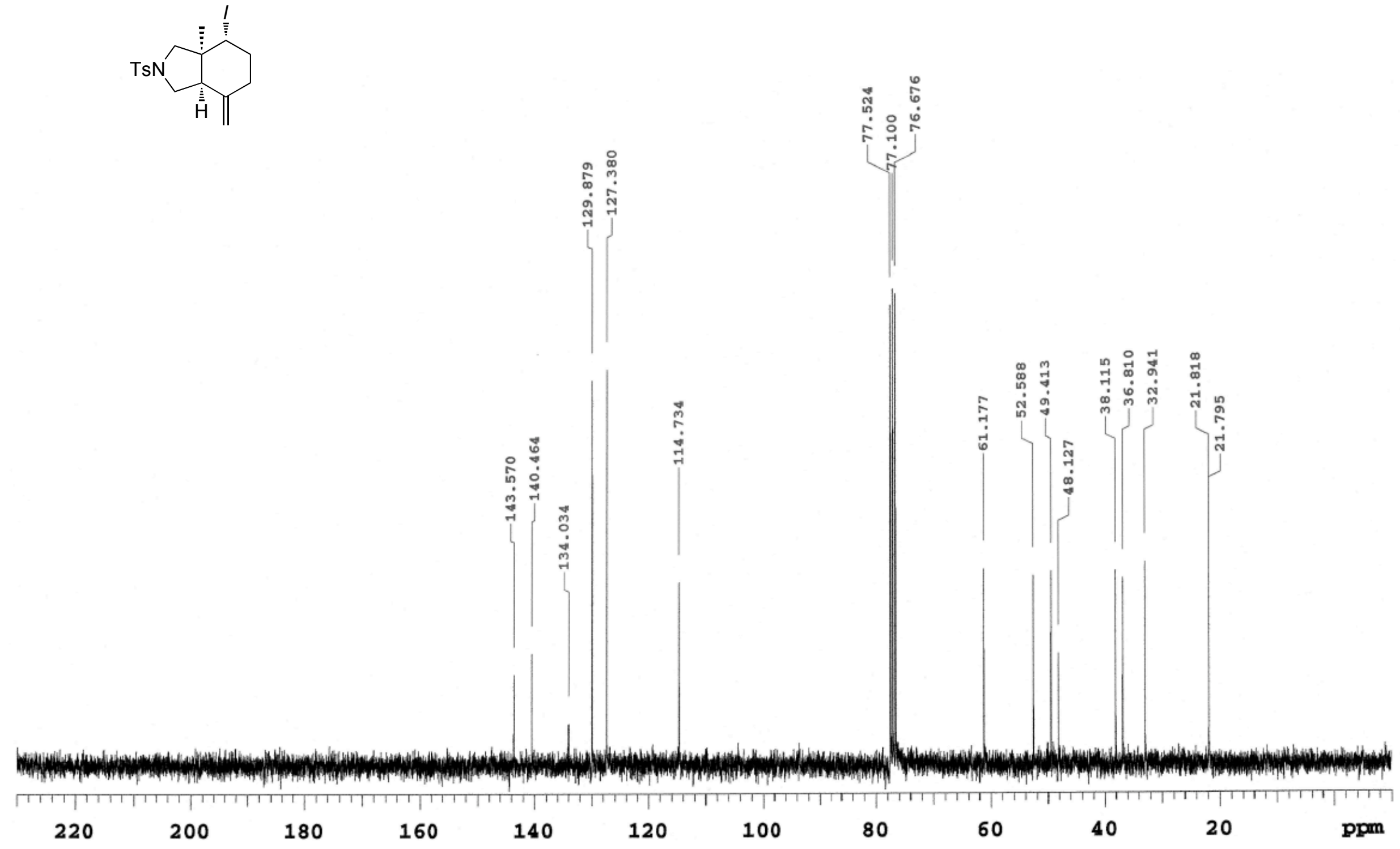




\section{${ }^{1} \mathrm{H}$ NMR Chart of $\mathbf{1 3 c}$}
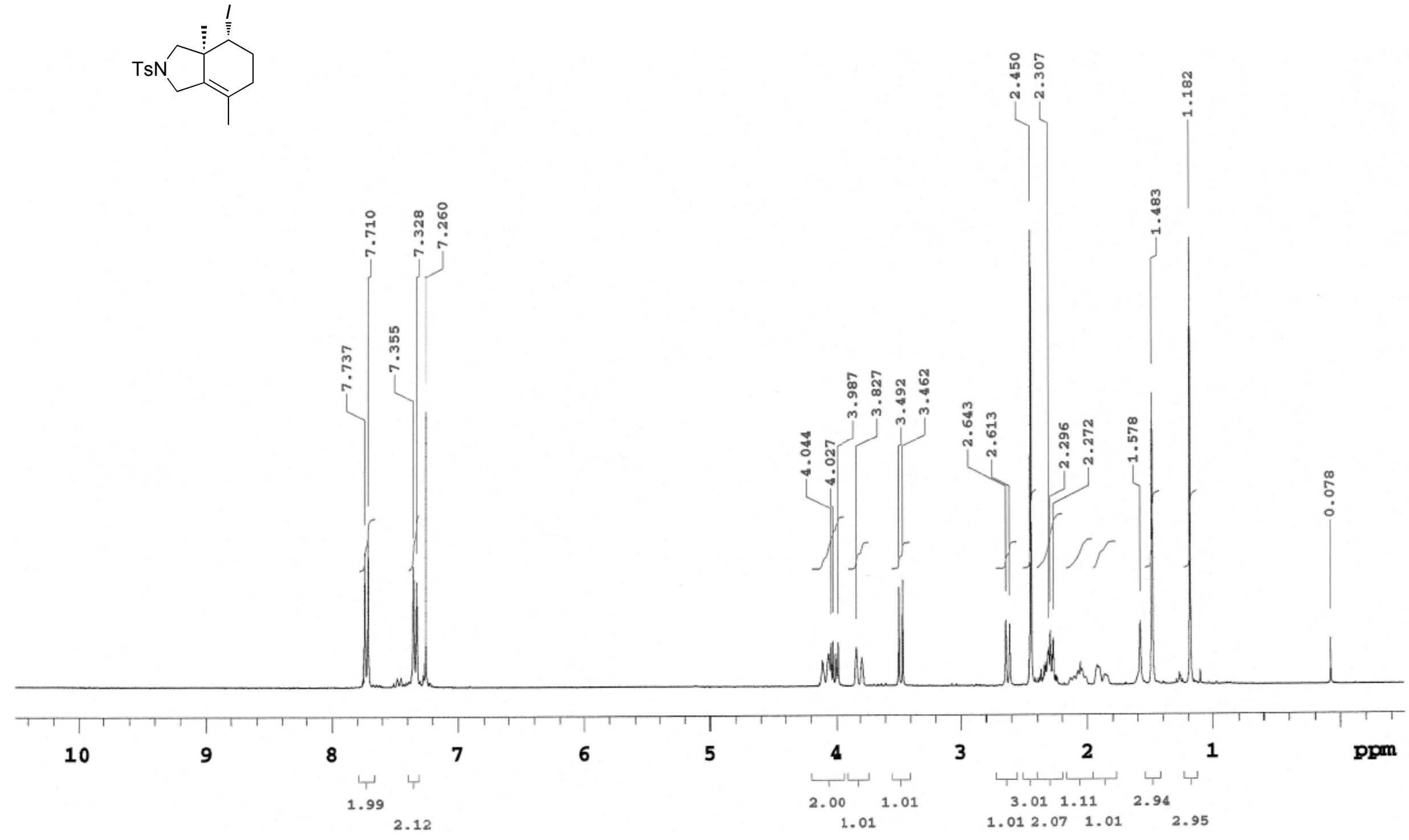
${ }^{13} \mathrm{C}$ NMR Chart of $\mathbf{1 3 c}$
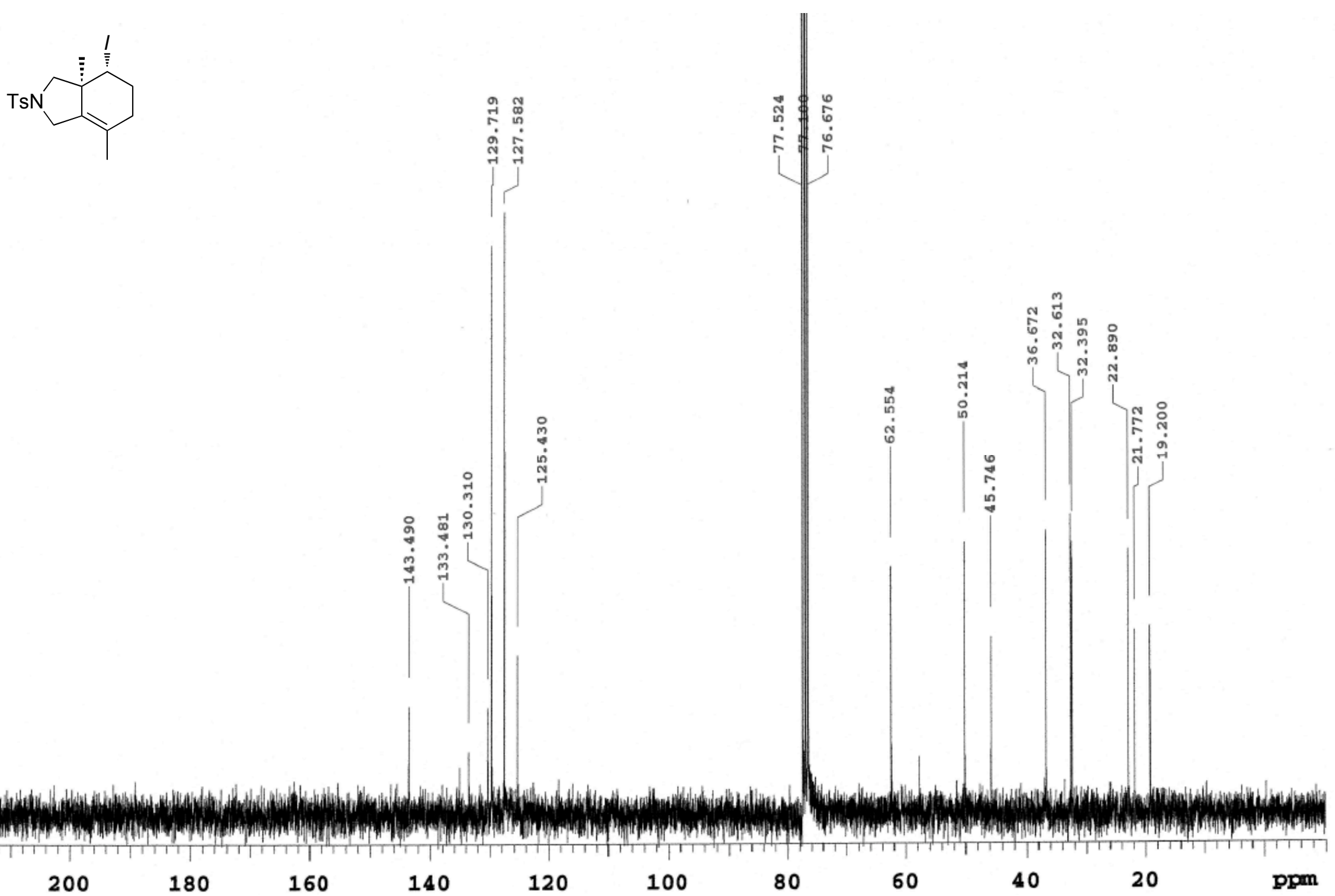
${ }^{1} \mathrm{H}$ NMR Chart of $N$-Tf-phosphoramide

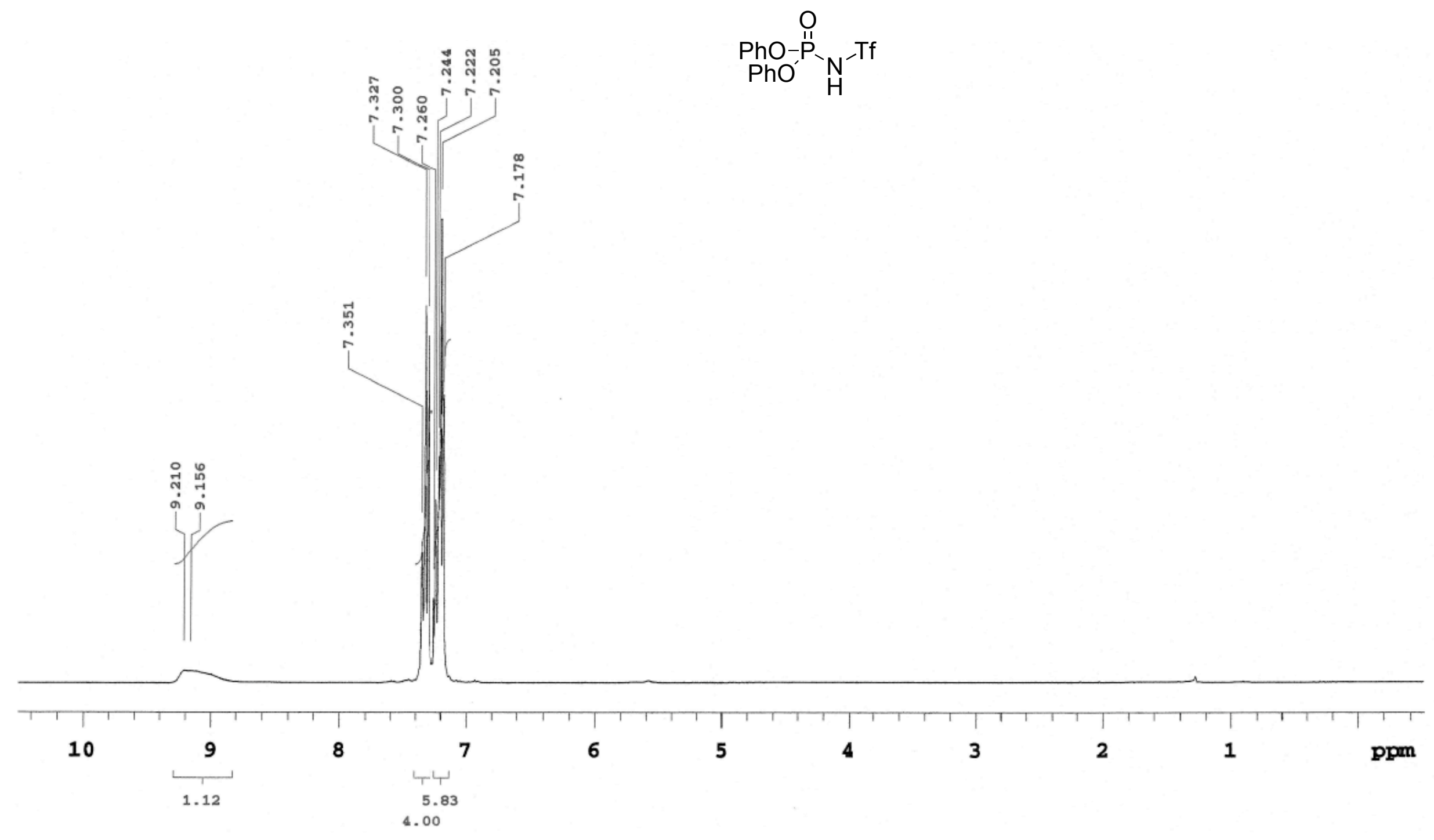


${ }^{13} \mathrm{C}$ NMR Chart of $N$-Tf-phosphoramide

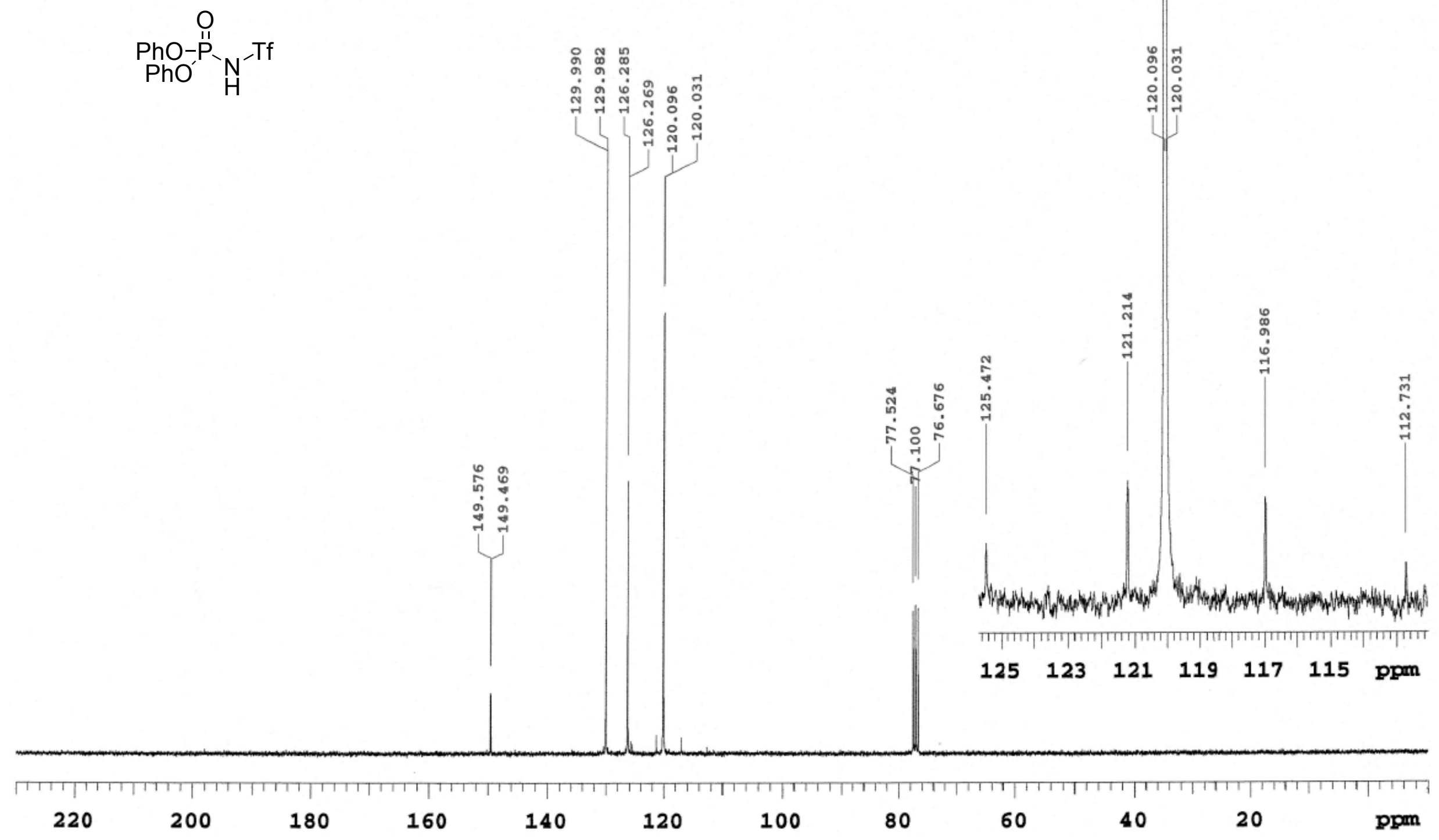


${ }^{19}$ F NMR Chart of $N$-Tf-phosphoramide
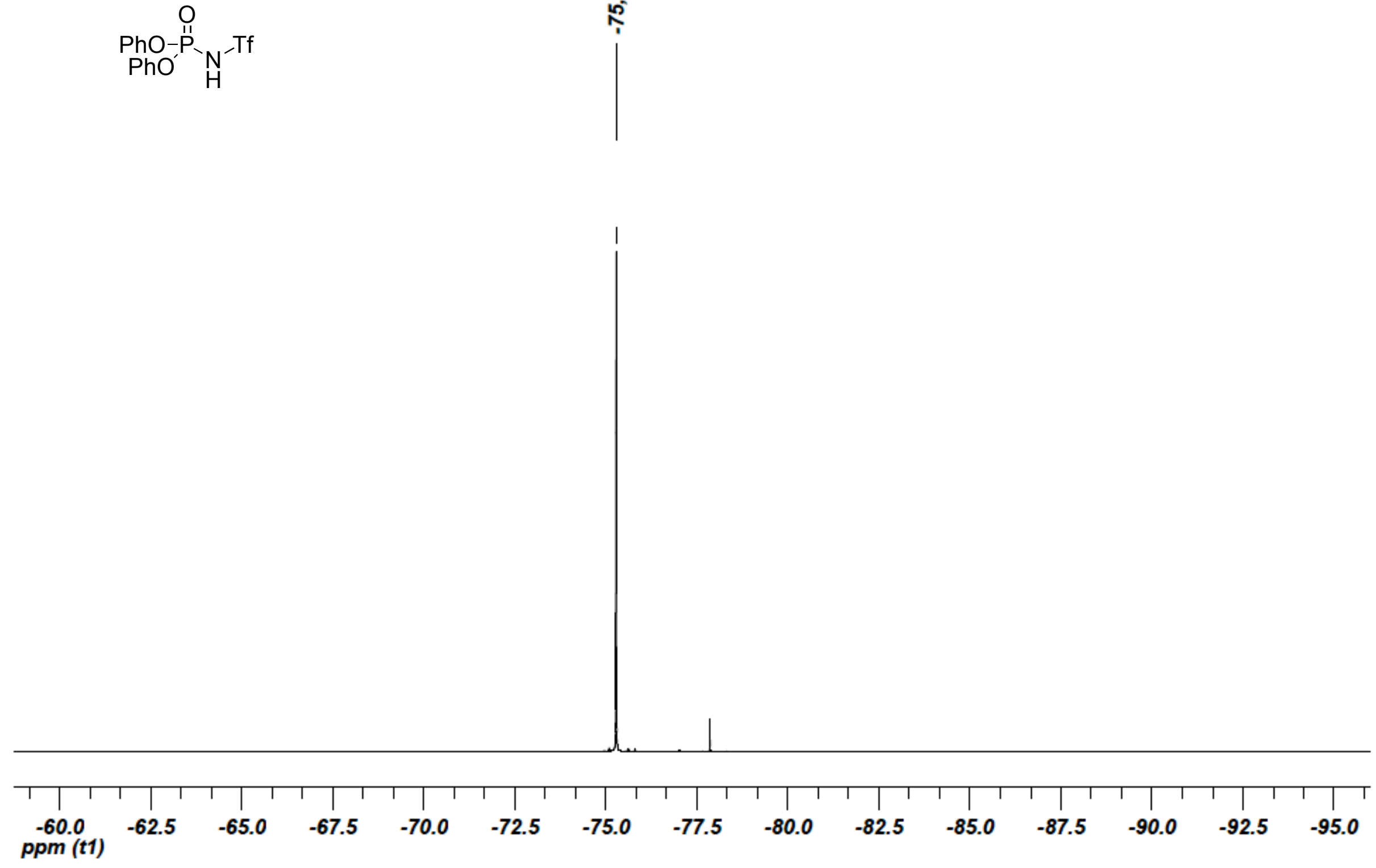
${ }^{31}$ P NMR Chart of $N$-Tf-phosphoramide

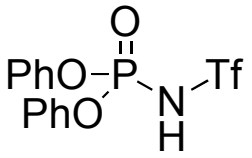
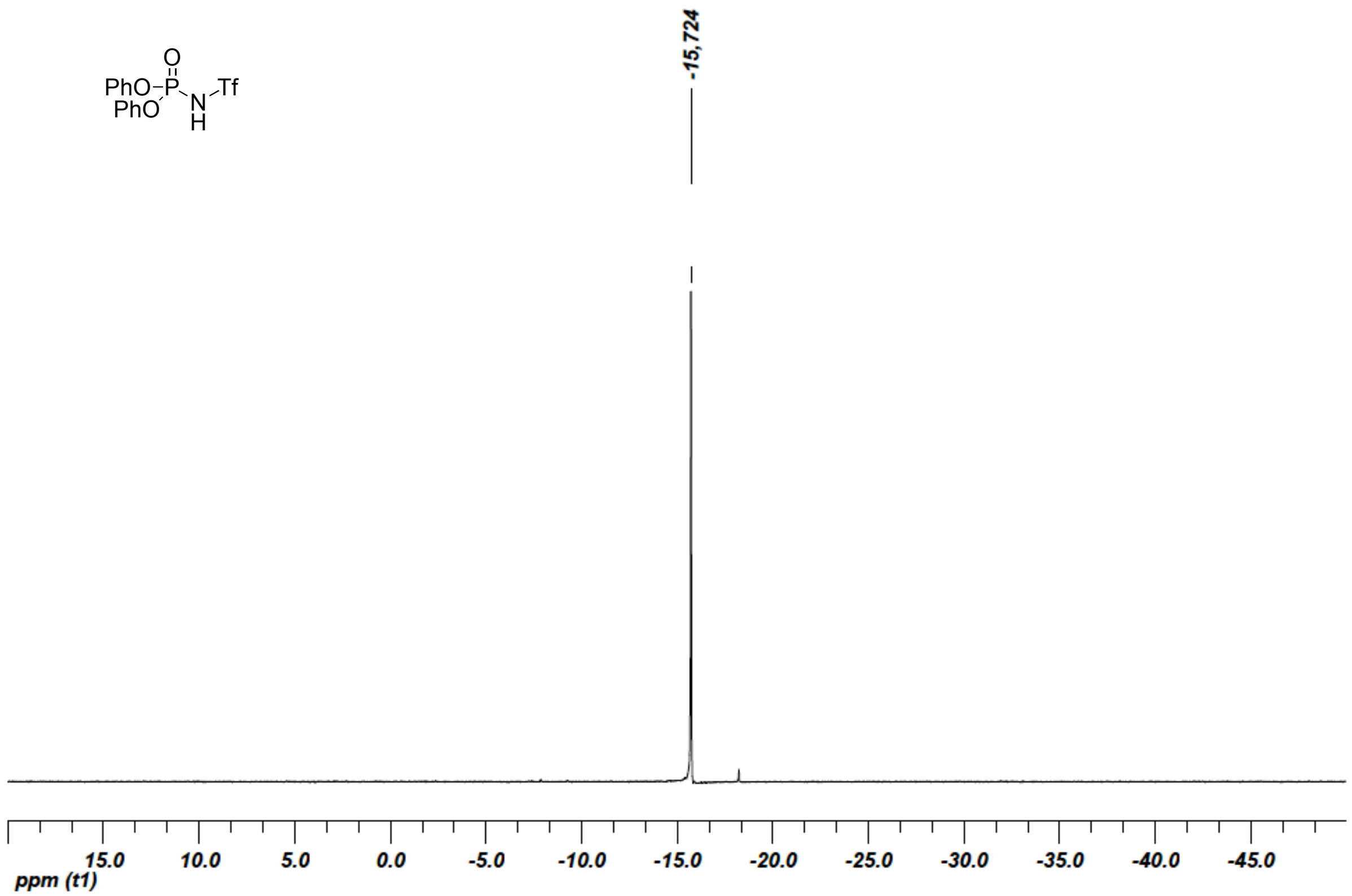
${ }^{1} \mathrm{H}$ NMR Chart of 14a
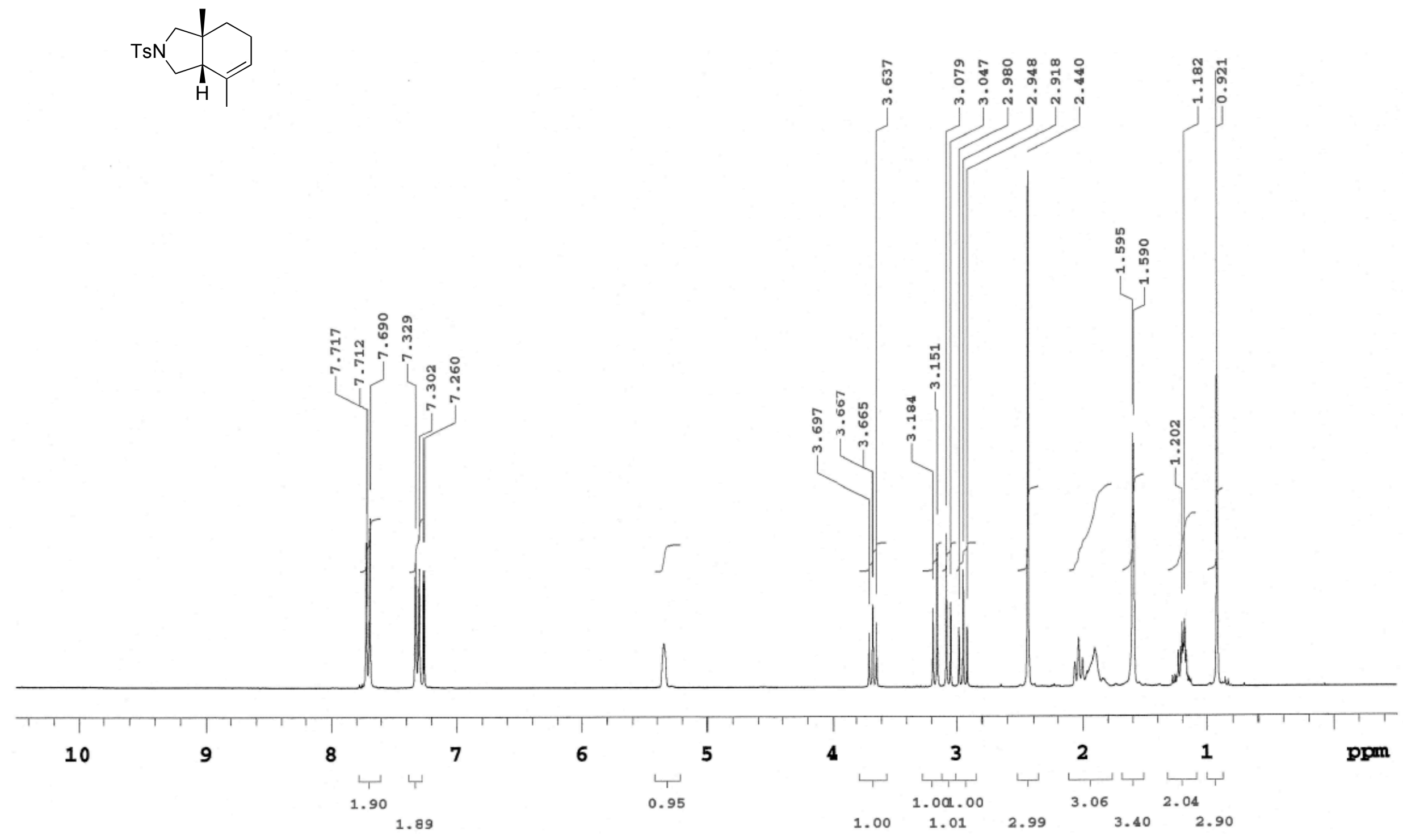
${ }^{13} \mathrm{C}$ NMR Chart of 14a
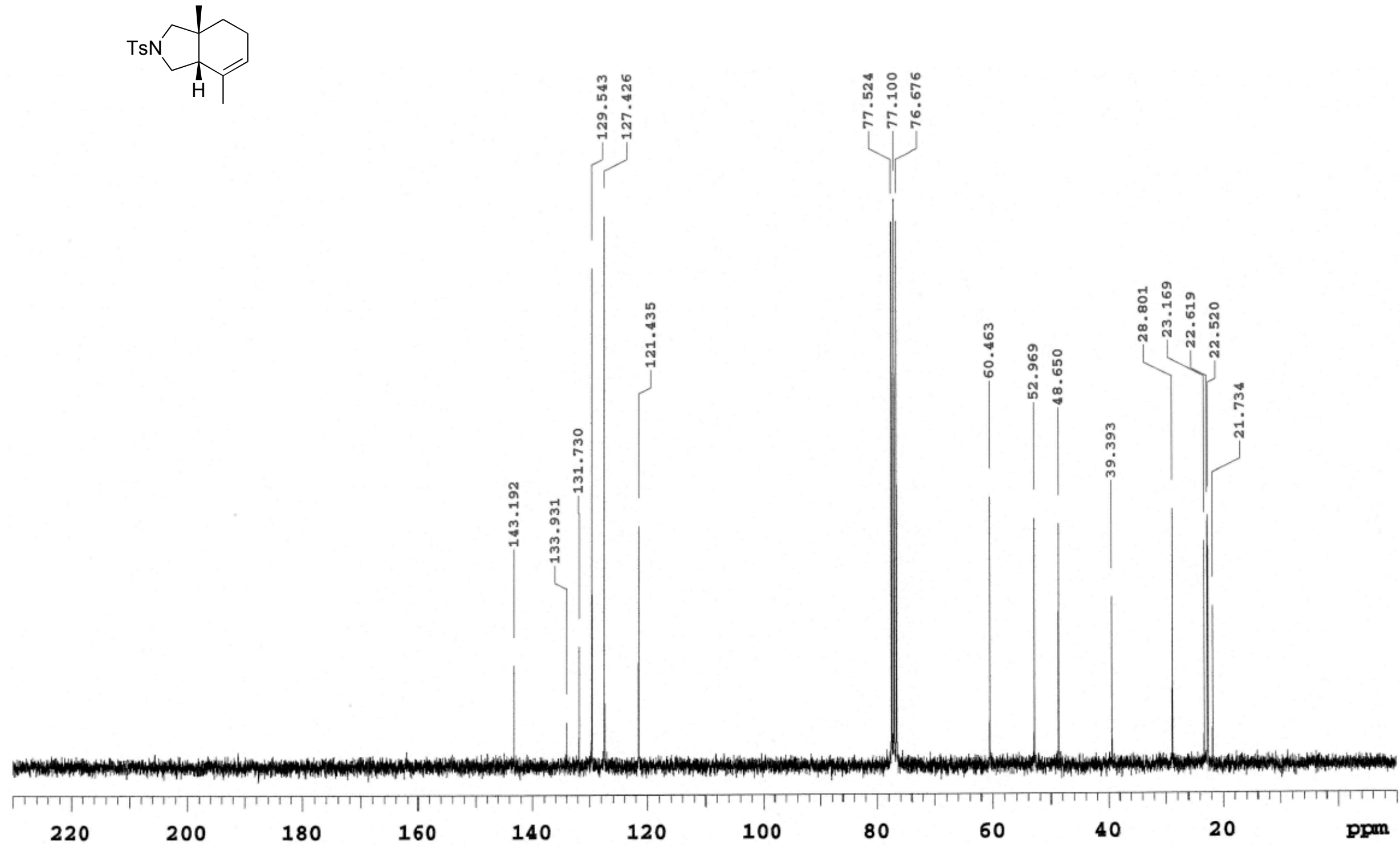
${ }^{1} \mathrm{H}$ NMR Chart of $\mathbf{1 4 b}$
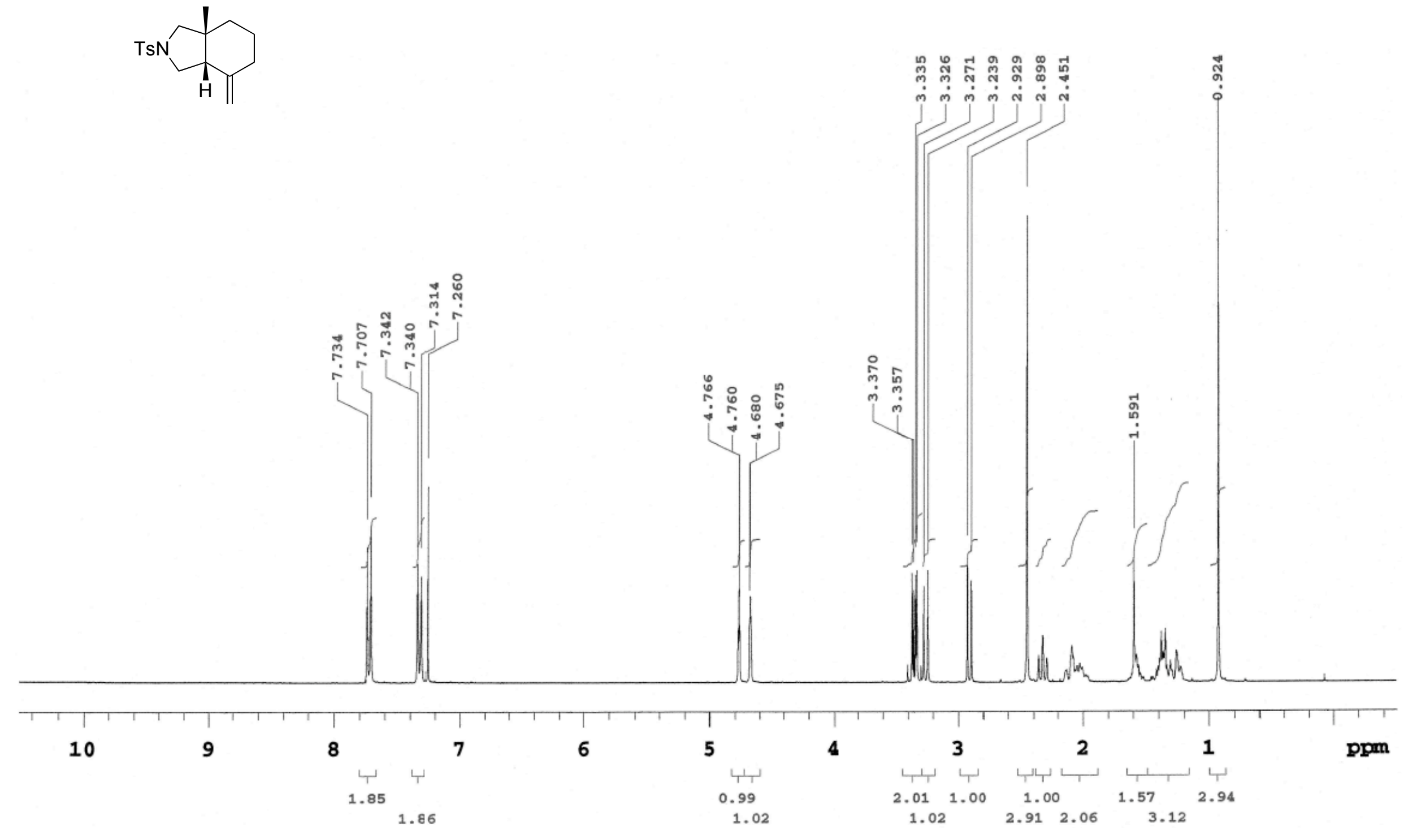
${ }^{13} \mathrm{C}$ NMR Chart of $\mathbf{1 4 b}$
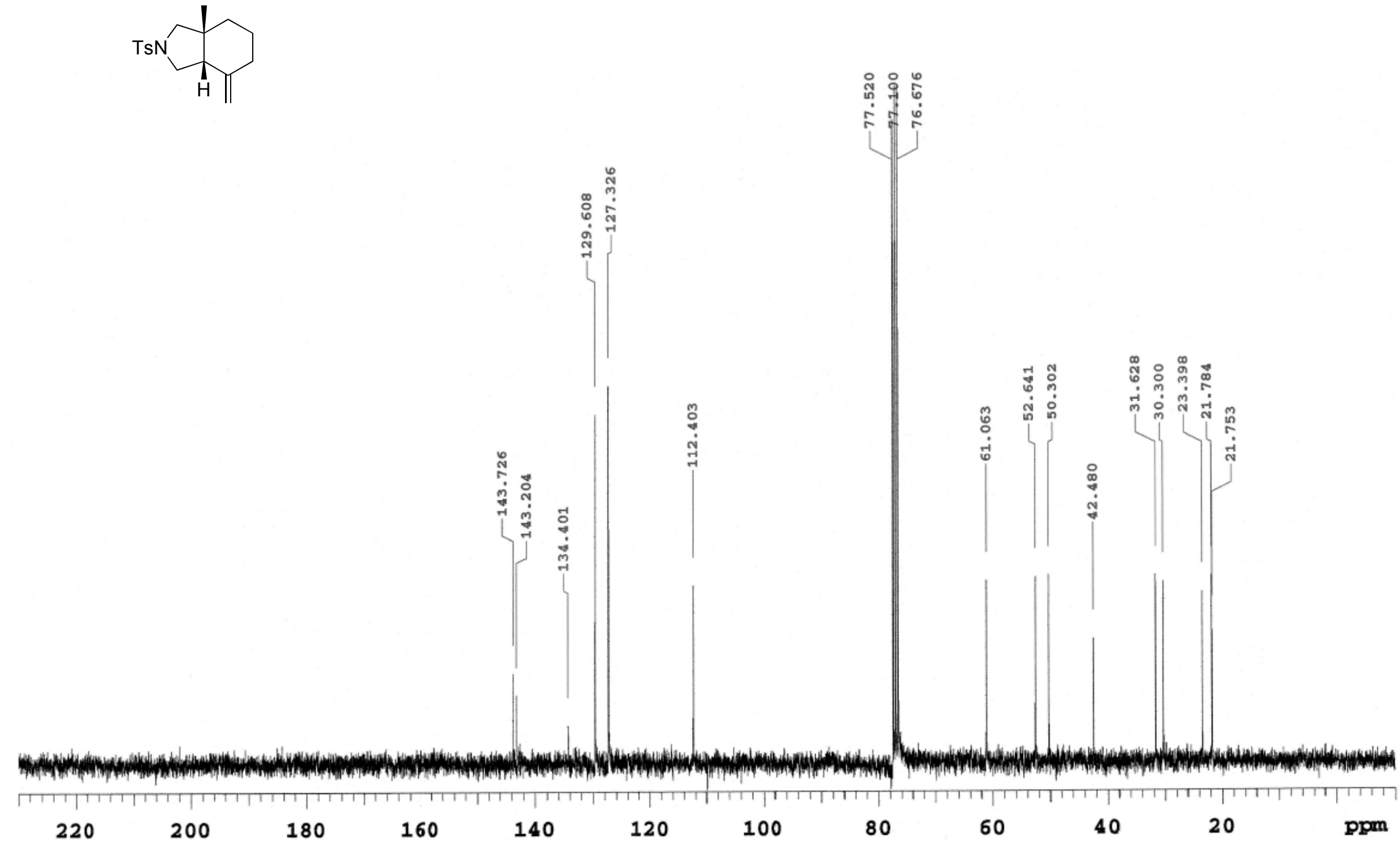